Homology, Homotopy and Applications, vol.11(2), 2009, pp.327-379

\title{
THE CONGRUENCE CRITERION FOR POWER OPERATIONS IN MORAVA E-THEORY
}

\author{
CHARLES REZK
}

\author{
(communicated by Nicholas J. Kuhn)
}

\begin{abstract}
We prove a congruence criterion for the algebraic theory of power operations in Morava $E$-theory, analogous to Wilkerson's congruence criterion for torsion free $\lambda$-rings. In addition, we provide a geometric description of this congruence criterion, in terms of sheaves on the moduli problem of deformations of formal groups and Frobenius isogenies.
\end{abstract}

\section{Introduction}

The purpose of this paper is to prove a congruence criterion for the algebraic theory of power operations acting on the homotopy of a $K(n)$-local commutative $E$ algebra spectrum, where $E$ denotes a spectrum of "Morava $E$-theory" at height $n$. This criterion is best understood as being a higher chromatic analogue of Wilkerson's congruence criterion for $\lambda$-rings.

\subsection{Algebraic theories of power operations}

By an "algebraic theory of power operations" for a commutative ring spectrum $A$, we mean an algebraic category which models all the algebraic structure which naturally adheres to $\pi_{*} A$, the homotopy groups of $A$. As an exemplar of this notion, consider the description of the natural operations on the homotopy of a commutative $H \mathbb{F}_{p}$-algebra spectrum, as given by McClure (see [BMMS86] especially §IX.2). In this work, it is shown (in modern language) that if $A$ is a commutative $H \mathbb{F}_{p}$-algebra, then $\pi_{*} A$ is an algebra for a certain monad $C$ on graded $\mathbb{F}_{p}$-vector spaces. Furthermore, an algebraic description for $C$-algebras is provided: a $C$-algebra amounts to a graded commutative $\mathbb{F}_{p}$-algebra, together with the structure of a module over the May-DyerLashof algebra, which (i) is compatible with multiplication, in the sense of having a suitable Cartan formula, and which (ii) satisfies an "instability" relation, which includes the fact that the "top" Dyer-Lashof operation is equal to the $p$ th power map. That this is the right answer is justified by the existence of a natural isomorphism $C\left(\pi_{*} M\right) \approx \pi_{*}(\mathbb{P} M)$, where $M$ is an $H \mathbb{F}_{p}$-module and $\mathbb{P}$ is the free $H \mathbb{F}_{p^{-}}$-algebra functor.

The author was supported under NSF grant DMS-0505056.

Received February 17, 2009, revised October 18, 2009; published on December 2, 2009.

2000 Mathematics Subject Classification: 55S25, 55S12, 14 L05.

Key words and phrases: power operation, Morava $E$-theory.

This article is available at http://intlpress.com/HHA/v11/n2/a16

Copyright (C) 2009, International Press. Permission to copy for private use granted. 
In this paper, we use the work of Ando, Hopkins and Strickland to develop an analogous theory for the homotopy of a $K(n)$-local commutative algebra over a Morava $E$-theory spectrum. Let $E$ denote the cohomology theory associated to the universal deformations of a height $n$ formal group $G_{0}$ over a perfect field $k$ of characteristic $p$. There is a monad $\mathbb{T}$ on the category $\operatorname{Mod}_{E_{*}}$ of graded $E_{*}=\pi_{*} E$-modules such that:

1. $\mathbb{T} E_{*} \approx \bigoplus_{m \geqslant 0} E_{*}^{\wedge} B \Sigma_{m}$, where $E_{*}^{\wedge}(-)$ denotes $K(n)$-localized homology;

2. $\mathbb{T}\left(M_{*} \oplus N_{*}\right) \approx \mathbb{T} M_{*} \otimes_{E_{*}} \mathbb{T} N_{*}$ for any $E_{*}$-modules $M_{*}$ and $N_{*}$;

3. There is a natural map $\mathbb{T}\left(\pi_{*} M\right) \rightarrow \pi_{*} \mathbb{P} M$ for $E$-module spectra $M$, where $\mathbb{P} M$ denotes the free commutative $E$-algebra on $M$. Furthermore, this map induces an isomorphism $\mathbb{T}\left(\pi_{*} M\right) \approx\left[\pi_{*} \mathbb{P} M\right]_{\mathfrak{m}}^{\wedge}$, where $N_{\mathfrak{m}}^{\wedge}$ denotes completion of an $E_{*^{-}}$ module $N$ with respect to the maximal ideal $\mathfrak{m} \subset \pi_{0} E$.

The category of algebras for this monad is denoted $\mathrm{Alg}_{\mathbb{T}}^{*}$; the monad $\mathbb{T}$ and its category of algebras are described in $\S \S 3$ and 4 .

\subsection{Description of $\mathrm{Alg}_{\mathbb{T}}^{*}$}

The next goal is to give a workable description of $\mathrm{Alg}_{\mathbb{T}}^{*}$. Every object of $\mathrm{Alg}_{\mathbb{T}}^{*}$ is (in particular) a graded commutative ring; it is also a graded right-module for a certain associative ring $\Gamma$, which we may call a "Dyer-Lashof algebra" (by analogy with the May-Dyer-Lashof algebra for ordinary $\bmod p$ homology).

The ring $\Gamma$ is defined in $\S 6$; effectively, $\Gamma$ is the ring of endomorphisms of the forgetful functor $\mathrm{Alg}_{\mathbb{T}}^{*} \rightarrow \mathrm{Ab}$ which sends an $\mathbb{T}$-algebra to its degree 0 part, viewed as an abelian group. Explicitly, $\Gamma$ is a direct sum of the $E_{0}$-linear duals of the rings $E^{0} B \Sigma_{p^{k}} /($ transfers $)$.

There is a ring homomorphism $\eta: E_{0} \rightarrow \Gamma$ (the image of which is not typically central). The ring $\Gamma$ is very nearly a Hopf algebra (more precisely, it is a "twisted bialgebra"; see $§ 5$ ), so that the category of $\Gamma$-modules admits a symmetric monoidal structure $\otimes: \operatorname{Mod}_{\Gamma}^{*} \times \operatorname{Mod}_{\Gamma}^{*} \rightarrow \operatorname{Mod}_{\Gamma}^{*}$, in such a way that the underlying $E_{*}$-module of $M \otimes N$ is precisely $M \otimes_{E_{*}} N$.

Let $\operatorname{Alg}_{\Gamma}^{*}$ denote the category of commutative monoid objects in graded $\Gamma$-modules. There is a forgetful functor $U: \operatorname{Alg}_{\mathbb{T}}^{*} \rightarrow \operatorname{Alg}_{\Gamma}^{*}$. The first main result in this paper describes the essential image of the restriction of $U$ to torsion free objects. Before stating the result, we consider the motivating example.

Example 1.1. Let $E$ be $p$-adic $K$-theory. In this case, $E_{0} \approx \mathbb{Z}_{p}$, and $\Gamma \approx \mathbb{Z}_{p}[\psi]$. The element $\psi \in \Gamma$ corresponds to the $p$ th Adams operation in the $K$-theory of a space. A $\Gamma$-algebra is precisely a $\psi$-ring, i.e., a graded commutative $\mathbb{Z}_{p}$-algebra $B$ together with a ring homomorphism $\psi: B \rightarrow B$. In this case, the structure of $\mathrm{Alg}_{\mathbb{T}}^{*}$ can also be completely understood, using the work of McClure in [BMMS86]. In particular, an object in $\mathrm{Alg}_{\mathbb{T}}^{*}$ is essentially what Bousfield [Bou96] calls a $\mathbb{Z} / 2$-graded $\theta$-ring. Thus, an object $\mathrm{Alg}_{\mathbb{T}}^{*}$ is strictly commutative graded $\mathbb{Z}_{p}$-algebra $B_{*}$, together with a function $\theta: B_{*} \rightarrow B_{*}$ which satisfies certain axioms. The operation $\psi$ is recovered from $\theta$ using the identity

$$
\psi(x)=x^{p}+p \theta(x)
$$

for all $x \in B_{0}$. The "Wilkerson criterion" states that a torsion free $\psi$-ring $B$ admits the structure of a $\theta$-ring (necessarily uniquely), if and only if $\psi(x) \equiv x^{p} \bmod p B$ 
for all $x \in B$. (This result is a " $p$-typicalization" of the original theorem of Wilkerson [Wil82], which characterizes the torsion free $\lambda$-rings in terms of congruences on the Adams operations at all primes.)

Our first result is a generalization of Wilkerson's criterion. Its statement involves a representative $\sigma \in \Gamma$ of a certain conjugacy class in $\Gamma / p \Gamma$, which is described in $\S 10.2$. We say that a graded $\Gamma$-algebra $B$ satisfies the congruence condition if for all $x \in B_{0}$,

$$
x \sigma \equiv x^{p} \bmod p B .
$$

Theorem A. An object $B \in \operatorname{Alg}_{\Gamma}^{*}$ which is p-torsion free admits the structure of a $\mathbb{T}$-algebra (necessarily uniquely), if and only if it satisfies the congruence condition.

The proof of Theorem A is completed in $\S 9$.

\subsection{Interpretation in terms of formal groups}

The second result of this paper gives a reinterpretation of the above theorem in terms of formal groups, and in doing so explains the significance of the element $\sigma \in \Gamma$.

Fix a perfect field $k$ of characteristic $p>0$, and a formal group $G_{0}$ of finite height over $k$. Let $E$ be the Morava $E$-theory associated to the universal deformation of $G_{0}$ in the sense of Lubin-Tate.

Given a complete local ring $R$, there is a category $\operatorname{Def}_{R}$, whose objects are deformations of $G_{0}$ to $R$, and whose morphisms are isogenies of formal groups which are "deformations of a power of Frobenius"; that is, morphism are isogenies which are identified with some power of the Frobenius isogeny when we base change to the residue field of $R$. The collection of categories $\operatorname{Def}_{R}$ for suitable rings $R$ and base change functors $f^{*}: \operatorname{Def}_{R} \rightarrow \operatorname{Def}_{R^{\prime}}$ describe a kind of moduli problem. We let $\mathrm{Sh}(\mathrm{Def}, \mathrm{Alg})^{*}$ denote the category of quasi-coherent sheaves of graded commutative $\mathcal{O}$-algebras over Def. An object $A$ of this category is (modulo some issues of grading) a pseudonatural transformation of pseudofunctors Def $\rightarrow \mathrm{Alg}^{*}$; more concretely, an object $A$ consists of data $\left\{A_{R}, A_{f}\right\}$, where for each ring $R$ there is a functor $A_{R}: \operatorname{Def}_{R} \rightarrow \operatorname{Alg}_{R}$, and for each local homomorphism $f: R \rightarrow R^{\prime}$ a natural isomorphism $A_{f}: A_{R^{\prime}} f^{*} \rightarrow f^{*} A_{R}$, satisfying a collection of coherence relations. The precise definitions of $\operatorname{Def}_{R}$ and $\mathrm{Sh}(\mathrm{Def}, \mathrm{Alg})^{*}$, including correct treatment of the grading, are given in $\S 11$.

We say that a quasi-coherent sheaf $A$ satisfies the Frobenius congruence if (roughly), for every $\mathbb{F}_{p}$-algebra $R$ and every object $G \in \operatorname{Def}(R)$, we have

$$
A_{R}\left(G \stackrel{\text { Frob }}{\longrightarrow} \phi^{*} G\right)=\left(A_{R}(G) \stackrel{\text { Frob }}{\longrightarrow} \phi^{*} A_{R}(G)\right),
$$

where "Frob" denotes the relative Frobenius isogeny on formal groups (on the lefthand side) and on $R$-algebras (on the right-hand side). (See $\S 11.10$ for the precise condition.)

Theorem B. There is an equivalence of categories $\mathrm{Sh}(\mathrm{Def}, \mathrm{Alg})^{*} \approx \mathrm{Alg}_{\Gamma}^{*}$. Under this equivalence, sheaves which satisfy the Frobenius congruence exactly correspond to graded $\Gamma$-algebras which satisfy the congruence condition. 
As described below, the equivalence of categories of Theorem B is well-known to experts in this area, and amounts to an interpretation of some theorems of Strickland. The proof of Theorem B is completed in $\S 12$.

\subsection{The work of Ando, Hopkins, and Strickland}

The structure of power operations on Morava E-theory is largely understood, thanks to work of Matt Ando, Mike Hopkins, and Neil Strickland. In particular, the hard results that underlie the version of the theory I will describe are theorems of Neil Strickland, and are proved in [Str97] and [Str98] (the latter corrected in [Str99]); this work in turn uses crucially some results of Kashiwabara [Kas98]. Unfortunately, there is no complete statement yet in print of the picture of operations on $E$-algebra spectra. Strickland provides a very brief sketch in [Str97, §14]. The three authors describe a version of this story in [AHS04]; see especially the material on "descent for level structures" [AHS04, 111 , which has informed $\S 11$ in this paper. Unfortunately, the level structure approach of [AHS04] is not convenient for describing the congruence condition.

Their unpublished work has some overlap with what we discuss in this paper. In particular, they constructed the algebra $\Gamma$ and perceived the equivalence of $\operatorname{Alg}_{\Gamma} \approx$ $\mathrm{Sh}$ (Def, Alg) of Theorem B (a large part of this is accomplished in Strickland's papers). They also understood that the difference between the categories $\mathrm{Alg}_{\mathbb{T}}$ and $\operatorname{Alg}_{\Gamma}$ was precisely an issue of understanding certain congruences, and that these congruences were generated by ones which are detected in the $E$-homology of the classifying space $B \Sigma_{p}$. The precise economical statement of Theorem $\mathrm{A}$ is new, as is the treatment of gradings.

\subsection{Treatment of gradings}

We should note that we deal with gradings in a somewhat novel way. Since Morava $E$-theory is an even periodic theory, we can regard the homotopy groups of a $K(n)$ local commutative $E$-algebra spectrum as being a $\mathbb{Z} / 2$-graded $\pi_{0} E$-module, rather than a $\mathbb{Z}$-graded $\pi_{*} E$-module. This would not be a viable procedure, except for the fact that we can modify the tensor product structure on $\mathbb{Z} / 2$-graded modules by "twisting" with the module $\omega=\pi_{2} E$ (see $\S 2$ for the precise formulation). One can think of our twisted $\mathbb{Z} / 2$-graded category as obtained by adjoining an "odd squareroot" of $\omega$ to a tensor category. This point of view turns out to be very convenient for dealing with power operations, and we believe it is worthy of attention.

\subsection{Completion}

There is a piece of structure on the homotopy $\pi_{*} B$ of a $K(n)$-local commutative $E$-algebra spectrum $B$ which is not encoded in our algebraic model $\mathrm{Alg}_{\mathbb{T}}^{*}$; namely, the fact that $\pi_{*} B$ is usually complete with respect to the maximal ideal of $\pi_{0} E$ (or more precisely, that $\pi_{*} B$ is always $L_{0}$-complete, see $\left.\S 3.3\right)$. It is fair to say that a defect of this paper is that we do not handle completion issues very well; most of the time, we sidestep the question. We hope to address these matters at some other time.

\subsection{Calculations}

As a companion piece to this paper, I have made available calculations of the structure of $\Gamma$ and $\operatorname{Alg}_{\mathbb{T}}^{*}$ in a particular case for height $n=2$ at the prime $p=2$ [Rez08]. 


\section{Acknowledgements}

I would like to thank Matt Ando and Mike Hopkins for helpful discussions relating to issues in this paper. I would especially like to thank Haynes Miller and MIT, for allowing me to teach a course on power operations as a visiting instructor there in the spring of 2006; the results of this paper were conjectured and proved while at MIT, and presented in the course I gave there.

\section{Twisted $\mathbb{Z} / 2$-graded categories}

In this section, we describe a procedure for constructing $\mathbb{Z} / 2$-graded additive tensor categories out of an additive tensor category, by formally adjoining an "odd squareroot" of an object $\omega$ of the original category. This procedure will be used in the rest of this paper to handle issues related to the "odd" degree gradings of Morava $E$-theory. It gives a very concise way to explain the graded nature of the objects in question, and we will use it both for odd degrees in Morava $E$-theory (see $\S 7$ ), and for the graded version of the category of sheaves on the deformation category (as in §11).

\subsection{Symmetric objects}

Let $(\mathcal{C}, \otimes, \mathbb{k})$ be an additive tensor category; that is, an additive category $\mathcal{C}$ equipped with a symmetric monoidal structure $\otimes$ with unit object $\mathbb{k}$, such that $\otimes$ distributes over finite sums.

Let $\tau_{M, N}: M \otimes N \rightarrow N \otimes M$ denote the interchange isomorphism of the symmetric monoidal structure on $\mathcal{C}$. Say that an object $\omega \in \mathcal{C}$ is symmetric if $\tau_{\omega, \omega}=\operatorname{id}_{\omega \otimes \omega}$; this is equivalent to requiring that the symmetric group act trivially on $\omega^{\otimes m}$ for all $m \geqslant 0$.

\subsection{Twisted tensor product}

Let $\mathcal{C}^{*}$ be the category of $\mathbb{Z} / 2$-graded objects of $\mathcal{C}$; an object of $\mathcal{C}^{*}$ is a pair $M^{*}=\left\{M^{0}, M^{1}\right\}$ of objects of $\mathcal{C}$, and a morphism $f: M^{*} \rightarrow N^{*}$ is a pair $f^{i}: M^{i} \rightarrow$ $N^{i}, i=0,1$, of morphisms of $\mathcal{C}$. We define a functor $\otimes: \mathcal{C}^{*} \times \mathcal{C}^{*} \rightarrow \mathcal{C}^{*}$ as follows. If $M^{*}$ and $N^{*}$ are objects in $\mathcal{C}^{*}$, then we define an object $M^{*} \otimes N^{*} \in \mathcal{C}^{*}$ by

$$
\begin{aligned}
& \left(M^{*} \otimes N^{*}\right)^{0} \stackrel{\text { def }}{=}\left(M^{0} \otimes N^{0}\right) \oplus\left(M^{1} \otimes N^{1} \otimes \omega\right), \\
& \left(M^{*} \otimes N^{*}\right)^{1} \stackrel{\text { def }}{=}\left(M^{0} \otimes N^{1}\right) \oplus\left(M^{1} \otimes N^{0}\right),
\end{aligned}
$$

where the tensor products on the right-hand side are taken in $\mathcal{C}$. We refer to this as the $\omega$-twisted tensor product on $\mathcal{C}^{*}$.

Let $\mathbb{k}=\{\mathbb{k}, 0\}$ as an object of $\mathcal{C}^{*}$; it serves as the unit object of the monoidal structure via the evident isomorphisms $\mathbb{k} \otimes M^{*} \approx M^{*} \approx M^{*} \otimes \mathbb{k}$. Define an interchange $\operatorname{map} \tau^{*}: M^{*} \otimes N^{*} \stackrel{\sim}{\longrightarrow} N^{*} \otimes M^{*}$ by

$$
\begin{aligned}
\tau^{0}:\left(M^{0} \otimes N^{0}\right) \oplus\left(M^{1} \otimes N^{1} \otimes \omega\right) & \rightarrow\left(N^{0} \otimes M^{0}\right) \oplus\left(N^{1} \otimes M^{1} \otimes \omega\right), \\
\left(m_{0} \otimes n_{0}, m_{1} \otimes n_{1} \otimes x\right) & \mapsto\left(\tau\left(m_{0} \otimes n_{0}\right),-\tau\left(m_{1} \otimes n_{1}\right) \otimes x\right), \\
\tau^{1}:\left(M^{0} \otimes N^{1}\right) \oplus\left(M^{1} \otimes N^{0}\right) & \rightarrow\left(N^{0} \otimes M^{1}\right) \oplus\left(N^{1} \otimes M^{0}\right), \\
\left(m_{0} \otimes n_{1}, m_{1} \otimes n_{0}\right) & \mapsto\left(\tau\left(m_{1} \otimes n_{0}\right), \tau\left(m_{0} \otimes n_{1}\right)\right) .
\end{aligned}
$$


(I have written these formulas using "element" notation, but they are easily converted into "arrow theoretic" formulas, meaningful in any additive tensor category $\mathcal{C}$.)

Finally, there is an associativity isomorphism

$$
\alpha:\left(M^{*} \otimes N^{*}\right) \otimes P^{*} \rightarrow M^{*} \otimes\left(N^{*} \otimes P^{*}\right)
$$

which I will not write out in detail. It is defined using the associativity and interchange isomorphisms for $\mathcal{C}$, with the interchange map used when needed to move the extra factor of $\omega$ into the "correct" position. For instance, $\left(\left(M^{*} \otimes N^{*}\right) \otimes P^{*}\right)^{0}$ contains a summand of the form $\left(M^{1} \otimes N^{1} \otimes \omega\right) \otimes P^{0}$, while the corresponding summand of $\left(M^{*} \otimes\left(N^{*} \otimes P^{*}\right)\right)^{0}$ is $M^{1} \otimes\left(N^{1} \otimes P^{0} \otimes \omega\right)$; the map $\alpha$ maps one to the other by switching $\omega$ and $P^{0}$ using the interchange map $\tau$.

Proposition 2.1. If $\omega \in \mathcal{C}$ is a symmetric object, then the above structure makes $\mathcal{C}^{*}$ into an additive tensor category. The functor $\mathcal{C} \rightarrow \mathcal{C}^{*}$ defined by $M \mapsto\{M, 0\}$ identifies $\mathcal{C}$ with a full monoidal subcategory of $\mathcal{C}^{*}$.

Proof. The only delicate point is to check the commutativity of the pentagon which compares the associativity isomorphisms of four-fold tensor products; that this commutes makes essential use of the fact that $\omega$ is symmetric.

We will typically identify $\mathcal{C}$ with its essential image in $\mathcal{C}^{*}$ without comment.

\subsection{The odd square root of $\omega$}

Let $\omega^{1 / 2}$ denote the object of $\mathcal{C}^{*}$ defined by $\omega^{1 / 2}=\{0, \mathbb{k}\}$. Then $\omega^{1 / 2} \otimes \omega^{1 / 2} \approx$ $\{\omega, 0\} \approx \omega$. Furthermore, the interchange map $\tau^{*}$ on $\omega^{1 / 2} \otimes \omega^{1 / 2}$ is equal to - id. Every object $M^{*}$ of $\mathcal{C}^{*}$ is isomorphic to one of the form $M^{0} \oplus\left(M^{1} \otimes \omega^{1 / 2}\right)$, where $M^{0}, M^{1} \in \mathcal{C} \subset \mathcal{C}^{*}$. Thus we can think of $\mathcal{C}^{*}$ as the additive tensor category obtained from $\mathcal{C}$ by "adjoining an odd square-root" of $\omega$.

\subsection{Functors from a twisted $\mathbb{Z} / 2$-graded category}

Given an additive tensor category $\mathcal{C}$, and a symmetric object $\omega$ of $\mathcal{C}$, we define the groupoid $\operatorname{Sqrt}(\omega)$ of odd square-roots of $\omega$ as follows. The objects of $\operatorname{Sqrt}(\omega)$ are pairs $(\eta, f)$, where $\eta$ is an object of $\mathcal{C}$ such that $\tau_{\eta, \eta}=-\mathrm{id}_{\eta \otimes \eta}$, and $f: \eta \otimes \eta \rightarrow \omega$ is an isomorphism, and the morphisms $(\eta, f) \rightarrow\left(\eta^{\prime}, f^{\prime}\right)$ of $\operatorname{Sqrt}(\omega)$ are isomorphisms $g: \eta \rightarrow \eta^{\prime}$ such that $f^{\prime}(g \otimes g)=f$.

Now suppose that $\mathcal{C}$ is an additive tensor category with symmetric object $\omega$, that $\mathcal{D}$ is an additive tensor category, and that $F: \mathcal{C} \rightarrow \mathcal{D}$ is a symmetric monoidal functor; thus $F(\omega)$ is a symmetric object of $\mathcal{D}$. Let $\mathcal{C}^{*}$ denote the $\mathbb{Z} / 2$-graded tensor category obtained from $\mathcal{C}$ and $\omega$, and identify $\mathcal{C}$ with its essential image in $\mathcal{C}^{*}$. Let $\mathcal{G}$ denote the groupoid whose objects are additive symmetric monoidal functors $F^{*}: \mathcal{C}^{*} \rightarrow \mathcal{D}$ such that $\left.F^{*}\right|_{\mathcal{C}}=F$, and whose morphisms $F_{1}^{*} \rightarrow F_{2}^{*}$ are monoidal natural isomorphisms which restrict to the identity map over $\mathcal{C}$.

Proposition 2.2. There is an equivalence of categories $\mathcal{G} \rightarrow \operatorname{Sqrt}(F(\omega))$, defined by $F^{*} \mapsto\left(F^{*}\left(\omega^{1 / 2}\right), g\right)$, where

$$
g: F^{*}\left(\omega^{1 / 2}\right) \otimes F^{*}\left(\omega^{1 / 2}\right) \stackrel{\sim}{\longrightarrow} F^{*}\left(\omega^{1 / 2} \otimes \omega^{1 / 2}\right) \stackrel{F^{*}(f)}{\longrightarrow} F^{*}(\omega)
$$

is the composite of the coherence map of $\mathcal{D}$ and the map $F^{*}(f)$, where $f: \omega^{1 / 2} \otimes$ $\omega^{1 / 2} \rightarrow \omega$ is the tautological isomorphism in $\mathcal{C}^{*}$. 


\subsection{Examples}

Example 2.3. If $\omega=\mathbb{k}$, then $\mathcal{C}^{*}$ is just the "usual" $\mathbb{Z} / 2$-graded category of objects of $\mathcal{C}$.

Example 2.4. Suppose the symmetric object $\omega$ is also $\otimes$-invertible in $\mathcal{C}$, i.e., there exists an object $\omega^{-1} \in \mathcal{C}$ and an isomorphism $\omega \otimes \omega^{-1} \approx \mathbb{k}$. Then we can define a $\mathbb{Z}$-graded commutative ring object $R_{*}$ of $\mathcal{C}$ by $R_{2 k}=\omega^{\otimes k}$ and $R_{2 k+1}=0$ for all $k \in \mathbb{Z}$. It is straightforward to check that $\mathcal{C}^{*}$ is then equivalent to the additive tensor category of $\mathbb{Z}$-graded modules over $R_{*}$. This equivalence associates a $\mathbb{Z}$-graded $R_{*}$ module $M_{*}$ with the object $M^{*}=\left\{M_{0}, M_{-1}\right\} \approx M_{0} \oplus\left(M_{-1} \otimes \omega^{1 / 2}\right)$ in $\mathcal{C}^{*}$.

\section{6. $\quad E_{*}$-modules}

Let $E_{*}=\pi_{*} E$, the coefficient ring of an even periodic ring spectrum, and let $\omega=$ $\pi_{2} E$ viewed as an $E_{0}=\pi_{0} E$-module. We can, and will, identify the category $\operatorname{Mod}_{E_{*}}$ of $\mathbb{Z}$-graded $E_{*}$-modules with the $\mathbb{Z} / 2$-graded category $\operatorname{Mod}_{E_{0}}^{*}$, where $\omega=\pi_{2} E=$ $E^{0} S^{2}=\pi_{0} \Sigma^{-2} E$ is used as the symmetric object. Observe that under the equivalence of $\mathbb{Z}$-graded and $\mathbb{Z} / 2$-graded $E_{*}$-modules described above, $\pi_{*} \Sigma^{q} E \approx E_{*} S^{q}$ is naturally identified with $\omega^{-q / 2}$. The Künneth isomorphism $E_{*} S^{i} \otimes_{E_{*}} E_{*} S^{j} \rightarrow E_{*}\left(S^{i} \wedge S^{j}\right) \approx$ $E_{*}\left(S^{i+j}\right)$ produces a canonical isomorphism $\kappa: \omega^{-i / 2} \otimes \omega^{-j / 2} \rightarrow \omega^{-(i+j) / 2}$.

If $M=\left\{M^{0}, M^{1}\right\}$ is an object of $\operatorname{Mod}_{E_{0}}^{*}$, then we can recover the $\mathbb{Z}$-graded $E_{*^{-}}$ module $M_{*}$ associated to it by $M_{q}=\operatorname{Hom}_{\operatorname{Mod}_{E_{0}}^{*}}\left(\omega^{-q / 2}, M\right)$.

In the examples we have given above, the symmetric object $\omega$ was $\otimes$-invertible. Later in this paper, in $\S \S 6$ and 11 , we will consider $\omega$-twisted tensor categories in cases where the symmetric object $\omega$ is not $\otimes$-invertible. It is in these non-invertible cases that the formalism of twisted $\mathbb{Z} / 2$-graded tensor categories proves especially convenient.

\section{Morava $E$-theory and extended powers}

\subsection{Morava $E$-theory}

We fix for the rest of the paper a perfect field $k$ of characteristic $p$, and a formal group $G_{0}$ over $k$ of height $n$, with $1 \leqslant n<\infty$. Let $E$ denote the Morava $E$-theory associated to the universal deformation of the formal group $G_{0}$.

It is a theorem of Goerss, Hopkins, and Miller, that $E$ is a commutative $S$-algebra in an essentially unique way [GH04].

\section{2. $\quad E$-modules and $E_{*}$-modules}

Let $\operatorname{Mod}_{E}$ denote the category of $E$-module spectra as in [EKMM95], and let $h \operatorname{Mod}_{E}$ denote its homotopy category. We write $M \wedge_{E} N$ and $\underline{h o m}_{E}(M, N)$ for the smash product and function spectrum of $E$-module, and also for their derived versions on $h \operatorname{Mod}_{E}$.

We write $E_{0}=\pi_{0} E$ and $\omega=\pi_{2} E$ as an $E_{0}$-module.

Taking homotopy groups defines a functor $\pi_{*}: \operatorname{Mod}_{E} \rightarrow \operatorname{Mod}_{E_{*}}$. In what follows we are going to regard $\operatorname{Mod}_{E_{*}}$ as the $\mathbb{Z} / 2$-graded category described in $\S 2.6$, so that 
the functor $\pi_{*}$ is explicitly described by

$$
\pi_{*} M=\left\{\pi_{0} M, \pi_{-1} M\right\} .
$$

Recall that according to our conventions, there are natural isomorphisms $\pi_{*} \Sigma^{q} M \approx$ $\omega^{-q / 2} \otimes \pi_{*} M$ for $E$-modules $M$, for all $q \in \mathbb{Z}$. (The reader may prefer to regard $\operatorname{Mod}_{E_{*}}$ as the usual category of modules over a $\mathbb{Z}$-graded ring; doing so should not cause any confusion until $\S 6$.)

\subsection{The completion functor}

Let $K(n)$ denote the $n$th Morava $K$-theory spectrum.

Let $L: \operatorname{Mod}_{E} \rightarrow \operatorname{Mod}_{E}$ denote the Bousfield localization functor with respect to the homology theory on $E$-modules defined by smashing with the module $E \wedge$ $K(n)$. It comes equipped with a natural coaugmentation map $j: M \rightarrow L M$, which is a $K(n)$-homology equivalence. The localization functor $L$ descends to a functor $h \operatorname{Mod}_{E} \rightarrow h \operatorname{Mod}_{E}$ on the homotopy category of $E$-module spectra, which we also denote $L$. We have the following equivalent descriptions of $L$.

Proposition 3.1. We have the following equivalences of coaugmented functors of the form $h \operatorname{Mod}_{E} \rightarrow h \operatorname{Mod}_{S}$, where $h \operatorname{Mod}_{S}$ denotes the homotopy category of spectra:

1. $L \approx L_{K(n)}$, where $L_{K(n)}$ denotes Bousfield localization of spectra with respect to Morava $K$-theory.

2. $L \approx L_{F(n)}$, where $L_{F(n)}$ denotes Bousfield localization of spectra with respect to a type $n$-finite spectrum $F(n)$.

3. $L M \approx \operatorname{holim}\left(E \wedge M\left(i_{0}, \ldots, i_{n-1}\right)\right) \wedge_{E} M$, where $\left\{M\left(i_{0}, \ldots, i_{n-1}\right)\right\}$ denotes a certain inverse system of finite spectra, constructed so that

$$
\pi_{*} E \wedge M\left(i_{0}, \ldots, i_{n-1}\right) \approx E_{*} /\left(p^{i_{0}}, u_{1}^{i_{1}}, \ldots, u_{n-1}^{i_{n-1}}\right) .
$$

Proof. Statement (1) is [EKMM95, Prop. VIII.1.7].

Statements (2) and (3) follow from [HS99, Prop. 7.10]; the proof of (2) uses the fact that $E$ is an $L_{n}$-local spectrum, whence the underlying spectrum of every $E$-module is $K(n)$-local.

Because of the last equivalence in this list, we can think of $L$ as a "completion" functor.

\subsection{Derived functors of $\mathfrak{m}$-adic completion}

Thus, let $L_{s}: \operatorname{Mod}_{E_{*}} \rightarrow \operatorname{Mod}_{E_{*}}$ denote the $s$ th left derived functor of $M \mapsto M_{\mathfrak{m}}^{\wedge}$. There is a map $L_{0} M \rightarrow M_{\mathfrak{m}}^{\wedge}$, which is not generally an isomorphism since completion is not right exact.

Proposition 3.2. The functors $L_{s}$ vanish identically if $s>n$. If $M_{*}$ is either a flat $E_{*}$-module, or a finitely generated $E_{*}$-module, then $L_{0}\left(M_{*}\right) \approx\left(M_{*}\right)_{\mathfrak{m}}^{\wedge}$ and $L_{s}\left(M_{*}\right)=0$ for $s>0$.

Proof. The first statement is [HS99, Thm. A.2(d)]. The statement about flat modules follows from [HS99, Thm. A.2(b)], and the statement about finitely generated modules follows from [HS99, Thm. A.6(e)]. 
The functor $L_{0}: \operatorname{Mod}_{E_{*}} \rightarrow \operatorname{Mod}_{E_{*}}$ is equipped with natural transformations $M \stackrel{i}{\rightarrow}$ $L_{0} M \stackrel{j}{\rightarrow} M_{\mathfrak{m}}^{\wedge}$. In general, $i: L_{0} M \rightarrow M_{\mathfrak{m}}^{\wedge}$ is a surjection, but not an isomorphism, while $L_{0}(i)$ is an isomorphism. The groups $L_{s} M$ can be identified with certain local cohomology groups of the modules $M$, as described in [HS99, Appendix A].

Proposition 3.3 ([Hov08, Prop. 2.3]). There is a conditionally and strongly convergent spectral sequence of $E_{*}$-modules

$$
E_{2}^{s, t}=L_{s} \pi_{t} M \Longrightarrow \pi_{s+t} L M
$$

which vanishes for $s>n$.

As a consequence, we have

Corollary 3.4. The map $\pi_{*} M \rightarrow \pi_{*} L M$ factors through a natural transformation $L_{0} \pi_{*} M \rightarrow \pi_{*} L M$ of functors $h \operatorname{Mod}_{E} \rightarrow \operatorname{Mod}_{E_{*}}$, which is a natural isomorphism whenever $\pi_{*} M$ is flat.

For a spectrum $X$, the completed $E$-homology of $X$ is defined by $E_{*}^{\wedge}(X) \stackrel{\text { def }}{=}$ $\pi_{*} L(E \wedge X)$.

\subsection{Complete $E$-modules}

A complete $E$-module is an $E$-module $M$ which is $K$-local, i.e., one such that $j: M \rightarrow L M$ is an equivalence. Note that

(a) If $M$ and $N$ are $E$-modules, and $N$ is complete, then $\underline{\operatorname{hom}}_{E}(M, N)$ is a complete E-module.

(b) If $M$ and $N$ are complete $E$-modules, then $M \wedge_{E} N$ need not be complete. However, $L\left(M \wedge_{E} N\right)$ is a complete $E$-module, called the completed smash product.

\subsection{Finite and finite free modules}

We write $\underline{h o m}_{E}(M, N)$ for the function spectrum in $\operatorname{Mod}_{E}$.

Proposition 3.5. Let $M$ be an E-module spectrum. The following are equivalent:

(1) $\pi_{*} M$ is a finitely generated (resp. finitely generated free) $E_{*}$-module.

(2) $\pi_{*} \underline{\operatorname{hom}}_{E}(M, E)$ is a finitely generated (resp. finitely generated free) $E_{*}$-module.

If either of (1) or (2) hold, then $M \approx L M$ and $M \approx \underline{\operatorname{hom}}_{E}\left(\underline{\operatorname{hom}}_{E}(M, E), E\right)$.

In particular, if $X$ is a spectrum, $E^{*} X$ is finitely generated (resp. free) if and only if $E_{*}^{\wedge} X$ is so.

Proof. See [HS99, §8].

We say a module $M$ is finite if $\pi_{*} M$ is a finitely generated $E_{*}$-module. A module is finite if and only if it is contained in the thick subcategory of $h \operatorname{Mod}_{E}$ generated by $E$. According to the above proposition, finite modules are complete.

Say that a $E$-module $M$ is finitely generated and free, or finite free for short, if $\pi_{*} M$ is a finitely generated free $\pi_{*} E$-module. All such modules are equivalent to ones of the form $\bigvee_{i=1}^{k} \Sigma^{d_{i}} E$. Note that if $M$ and $N$ are finite (resp. finite free), then 
so are $M \wedge_{E} N$ and $\underline{\operatorname{hom}}_{E}(M, N)$. Also, retracts of finite free modules are also finite free.

Let $\operatorname{Mod}_{E}^{\mathrm{ff}}$ denote the full subcategory of $\operatorname{Mod}_{E}$ consisting finite free modules. Let $h \operatorname{Mod}_{E}^{\mathrm{ff}}$ denote the full subcategory of $h \operatorname{Mod}_{E}$ spanned by the finite free modules.

Proposition 3.6. The functor $\pi_{*}: h \operatorname{Mod}_{E}^{\mathrm{ff}} \rightarrow \operatorname{Mod}_{E_{*}}^{\mathrm{ff}}$, which associates to an E-module its homotopy groups, is an equivalence of categories.

Proof. A straightforward consequence of the observation that $h \operatorname{Mod}_{E}\left(\Sigma^{d} E, M\right) \approx$ $\pi_{d} M$.

\subsection{Flat modules}

Say that an $E$-module $M$ is flat if $\pi_{*} M$ is flat as a graded $E_{*}$-module. We will need the following analogue of Lazard's theorem on flat modules over a ring, which is a variant of an observation of Lurie [Lur07, §4.6].

Let $C$ be a category enriched over spaces. Say that $C$ is filtered if the following hold:

1. For every finite set of objects $X_{1}, \ldots, X_{k}$ in $C$, there exists an object $Y$ and morphism $X_{j} \rightarrow Y$ in $C$ for all $j=1, \ldots, k$.

2. For all $k \geqslant 0$, all $X, Y$ objects of $C$, and all maps $S^{k} \rightarrow C(X, Y)$, there exists a map $g: Y \rightarrow Z$ and a dotted arrow making the diagram

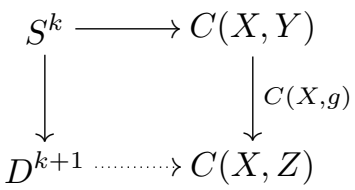

commute.

For a category $C$ enriched over spaces, let $\pi_{0} C$ denote the ordinary (enriched over sets) category with the same objects as $C$, whose morphisms are the sets of path components of the mapping spaces of $C$. If $C$ is filtered in the above sense, then the ordinary category $\pi_{0} C$ is filtered in the usual sense.

Proposition 3.7. An object $M \in \operatorname{Mod}_{E}$ is flat if and only if it is weakly equivalent to the homotopy colimit of some continuous functor $F: C \rightarrow \operatorname{Mod}_{E}^{\mathrm{ff}}$, where $C$ is filtered.

Proof. This is proved in much the same way as [Lur07, Thm. 4.6.19], though with changes of detail, since the notions of "flat" and "finite free" we use differ than the ones Lurie uses. We briefly sketch the ideas here.

For the if direction, it suffices to note that taking homotopy groups commutes with taking homotopy colimit over a filtered diagram.

For the only if direction, consider the comma category $\operatorname{Mod}_{E} / M$, which admits the structure of a topological closed model category. Choose a set $S$ of fibrant-andcofibrant representatives of weak equivalence classes of objects $(F, f: F \rightarrow M)$ for which $F$ is finite free, and let $C$ be the full topological subcategory of $\operatorname{Mod}_{E} / M$ spanned by $S$. Now one shows that if $M$ is flat, then $C$ is a filtered topological category in the sense described above. Given this, it is clear that $M$ is equivalent to the homotopy colimit of the canonical functor $C \rightarrow \operatorname{Mod}_{E}$. 


\subsection{Completed extended powers}

If $M$ is an $E$-module, the the $m$ th symmetric power is the quotient $\left(M^{\wedge}{ }_{E}^{m}\right)_{h \Sigma_{m}}$ of the $m$ th smash power by the evident symmetric group action. The free commutative $E$-algebra on $M$ is the coproduct $\bigvee_{m \geqslant 0} \mathbb{P}_{m}(M)$.

In this paper, we will deal mainly with the $m$ th extended powers; we write $\mathbb{P}_{m}(M) \stackrel{\text { def }}{=}\left(M^{\wedge_{E} m}\right)_{h \Sigma_{m}}$ for this; the extended power also passes to a functor on the homotopy category $h \operatorname{Mod}_{E}$, also denoted $\mathbb{P}_{m}$. We recall that, in the EKMM model for $S$-modules, if we choose a $q$-cofibrant model for the commutative $S$-algebra $E$, then the symmetric powers of cell cofibrant $R$-modules are homotopy equivalent to extended powers [EKMM95, III.5].

We write $\mathbb{P}(M)=\bigvee_{m \geqslant 0} \mathbb{P}_{m}(M)$. If $G \subseteq \Sigma_{m}$ is a subgroup, we write $\mathbb{P}_{G}(M) \stackrel{\text { def }}{=}$ $\left(M^{\wedge}{ }_{E} m\right)_{h G}$. The functor $\mathbb{P}$ defines a monad on the homotopy category of $E$-modules. We will assume that the reader is familiar with properties of these functors, for instance as described in [BMMS86, Ch. 1]. In particular, we note that $\mathbb{P}$ defines a monad on the the homotopy category $h \operatorname{Mod}_{E}$ of $E$-modules, and any commutative $E$-algebra results in an algebra for this monad.

Proposition 3.8. The functors $\mathbb{P}_{m}$ preserve $K$-homology isomorphisms. In particular, the map $\mathbb{P}_{m}(j): \mathbb{P}_{m}(M) \rightarrow \mathbb{P}_{m}(L M)$ is a K-homology isomorphism, and thus there is a natural isomorphism $L \mathbb{P}_{m}(j): L \mathbb{P}_{m} \rightarrow L \mathbb{P}_{m} L$ of functors on $h \operatorname{Mod}_{E}$. The functor $L \mathbb{P}: h \operatorname{Mod}_{E} \rightarrow h \operatorname{Mod}_{E}$ admits a unique monad structure with the property that $j$ is a map of monads.

Proof. The functors $\mathbb{P}$ are homology isomorphisms for any homology theory; the remaining statements are straightforward.

The goal of this section is to prove

Proposition 3.9. If $M$ is an E-module which is finite free, then $L \mathbb{P}_{m}(M)$ is also finite free.

This is well-known in the case that $\pi_{*} M$ is concentrated in even degree. (See [HKR00, Thm. D].)

Proposition 3.10. If $G$ contains a p-Sylow subgroup of $\Sigma_{m}$, then $\mathbb{P}_{G}(M) \rightarrow \mathbb{P}_{m}(M)$ admits a section for any $E$-module $M$.

Proof. If $G$ is a subgroup of a group $H$, with index prime to $p$, then the map of spectra $\Sigma_{+}^{\infty}(H / G)_{(p)} \rightarrow \Sigma_{+}^{\infty}(H / H)_{(p)}$ admits a retraction in the homotopy category of spectra equipped with a $H$ action.

Recall that $p$ is the characteristic of the residue field of $E_{*}$. Let $\rho_{C_{p}}$ denote the real regular representation of the cyclic group $C_{p}$, and let $B C_{p}^{c \rho_{C_{p}}}$ denote the Thom spectrum of the virtual representation $c \rho_{C_{p}}$, where $c \in \mathbb{Z}$.

Lemma 3.11. If $c \in \mathbb{Z}$, then $E_{*}^{\wedge} B C_{p}^{c \rho_{C_{p}}}$ is a finitely generated free $\pi_{*} E$-module. 
Proof. First suppose $c=0$. Then the cofiber sequence

$$
B C_{p} \approx S\left(\lambda^{\otimes p}\right) \rightarrow B S^{1} \rightarrow\left(B S^{1}\right)^{\lambda^{\otimes p}}
$$

associated to the universal line bundle $\lambda$ over $B S^{1}$, together with the Thom isomorphism for E-theory, give

$$
0 \leftarrow E^{*} B C_{p} \leftarrow E \llbracket x \rrbracket \stackrel{[p](x)}{\longleftarrow} E \llbracket x \rrbracket \leftarrow 0,
$$

and in particular since $[p](x) \equiv x^{p^{n}} \bmod \mathfrak{m}, E^{*} B C_{p}$ is free over $E_{*}$ on $1, \ldots, x^{p^{n}-1}$. Thus $\operatorname{hom}\left(\Sigma_{+}^{\infty} B C_{p}, E\right)$ is a finitely generated free $E$-module, and therefore so is $L\left(E \wedge \Sigma_{+}^{\infty} B C_{p}\right)$ by $(3.5)$.

The Thom isomorphism for $E$-theory immediately gives the result for even $c$, by identifying $2 d \rho_{C_{p}}$ with the complex bundle $d \rho_{C_{p}} \otimes \mathbb{C}$. It remains to check the case of odd $c$, and the Thom isomorphism allows us to reduce to the case $c=1$.

If $p$ is odd, then there is a splitting $\rho_{C_{p}} \approx \mathbb{R} \oplus \bar{\rho}_{C_{p}}$ of real $C_{p}$-representations, where the real representation $\bar{\rho}_{C_{p}}$ admits a complex structure. The result follows using the Thom isomorphism, since $E$ is complex orientable.

If $p=2$, then $\rho_{C_{2}} \approx \mathbb{R} \oplus \bar{\rho}_{C_{2}}$, and $\bar{\rho}_{C_{2}}$ is the sign representation, so that as spaces,

$$
B C_{2}^{\rho_{C_{2}}} \approx \Sigma B C_{2}^{\bar{\rho}_{C_{2}}} \approx \Sigma B C_{2} \text {. }
$$

Stably, the latter is a retract of $\Sigma\left(\Sigma_{+}^{\infty} B C_{2}\right)$, whose completed $E$-homology is finite free as noted above.

Proof of (3.9). Since $\left(S^{c}\right)_{h C_{p}}^{\wedge p} \approx B C_{p}^{c \rho_{C_{p}}}$, (3.11) implies that $L \mathbb{P}_{C_{p}}\left(\Sigma^{c} E\right)$ is finitely generated free. Thus (3.10) shows that $L \mathbb{P}_{p}(M)$ is a retract of $L \mathbb{P}_{C_{p}}(M)$, so that $L \mathbb{P}_{p}\left(\Sigma^{c} E\right)$ is finitely generated free.

The "binomial formula" for $\mathbb{P}_{p}$ says that

$$
\mathbb{P}_{p}(M \vee N) \approx \bigvee_{i+j=p} \mathbb{P}_{i}(M) \wedge_{E} \mathbb{P}_{j}(N),
$$

and since $\mathbb{P}_{i}(M)$ is a retract of $M^{\wedge_{E} i}$ by $(3.10)$, we conclude that $L \mathbb{P}_{p}(M)$ takes finite frees to finite frees.

We have that

$$
\mathbb{P}_{G} \mathbb{P}_{H}(M) \approx \mathbb{P}_{H \zeta G}(M) .
$$

If $\Sigma_{p}^{2 r}$ denotes the $r$-fold wreath power, then we have shown that $L \mathbb{P}_{\Sigma_{p}^{2 r}}=L \mathbb{P}_{p} \cdots \mathbb{P}_{p}$ preserves finite frees.

Finally, for $m \geqslant 0$ with $m=\sum a_{i} p^{i}$, with $a_{i} \in\{0, \ldots, p-1\}$, the group $\Sigma_{m}$ contains a subgroup $G=\prod_{i}\left(\Sigma_{p}^{2 i}\right)^{\times a_{i}}$, which acts on $\underline{m}$ in the evident way, and which contains a $p$-Sylow subgroup of $G$. By $(3.10), \mathbb{P}_{m}(\bar{M})$ is a retract of the smash product of finitely many $\mathbb{P}_{\Sigma_{p}^{i i}}(M)$, and therefore we are done.

Remark 3.12. The above proof shows a little bit more. Namely, if $M$ is a finite free module with $\pi_{*} M$ concentrated in even degree, then $\pi_{*} L \mathbb{P}_{m}(M)$ is also concentrated in even degrees. There is no corresponding result when $\pi_{*} M$ is concentrated in odd degree, although the proof of (3.9) implies the following result. 
Corollary 3.13. The graded module $\pi_{*} L \mathbb{P}_{p}\left(\Sigma^{c} E\right)$ is concentrated in odd degree if $c$ is odd.

We also note the following interesting consequence (a generalization of an observation of McClure).

Proposition 3.14. If $A$ is a $K(n)$-local commutative E-algebra, then the multiplication on $\pi_{*} A$ is strictly graded commutative, in the sense that if $x \in \pi_{q} A$ with $q$ odd, then $x^{2}=0$.

Proof. It is clear that we only need to prove something in the 2-local case. Let $f: \Sigma^{q} E \rightarrow A$ be the $E$-module map which represents $x$. Then $x^{2} \in \pi_{2 q} A$ is the image of an element in $\pi_{2 q} L \mathbb{P}_{2}\left(\Sigma^{q} E\right)$ under the map $L \mathbb{P}_{2}\left(\Sigma^{q} E\right) \rightarrow L \mathbb{P}_{2}\left(\Sigma^{q} A\right) \rightarrow A$. But $\pi_{*} L \mathbb{P}_{2}\left(\Sigma^{q} E\right)$ is concentrated in odd degree by (3.13).

\subsection{Power operations}

Let $R$ be a commutative $E$-algebra spectrum. For any space $X$ and any $m \geqslant 0$, we obtain an operation

$$
P_{m}: R^{0} X \rightarrow R^{0}\left(X \times B \Sigma_{m}\right),
$$

defined so that an $E$-module map $x: E \wedge \Sigma_{+}^{\infty} X \rightarrow R$ is sent to the composite

$$
E \wedge \Sigma_{+}^{\infty}\left(X \times B \Sigma_{m}\right) \rightarrow E \wedge \Sigma_{+}^{\infty} X_{h \Sigma_{m}}^{m} \approx \mathbb{P}_{m}\left(E \wedge \Sigma_{+}^{\infty} X\right) \stackrel{\mathbb{P}_{m}(x)}{\longrightarrow} \mathbb{P}_{m} R \rightarrow R
$$

The operation $P_{m}$ is called the $m$ th power operation. It has the property that $P_{m}(x y)=P_{m}(x) P_{m}(y)$. Since $E^{0} B \Sigma_{m}$ is a finite free $E_{0}$-module, there is an isomorphism $R^{0}\left(X \times B \Sigma_{m}\right) \approx R^{0} X \otimes_{E_{0}} E^{0} B \Sigma_{m}$.

If $R^{X}$ denotes the spectrum of functions from $\Sigma_{+}^{\infty} X$ to $R$, which is a commutative $E$-algebra, then the power operation $P_{m}:\left(R^{X}\right)^{0}(*) \rightarrow\left(R^{X}\right)^{0}\left(B \Sigma_{m}\right)$ coincides with power operation on $R^{0} X$.

Let

$$
J=\sum_{0<i<m} \operatorname{im}\left[R^{0}\left(X \times B\left(\Sigma_{i} \times \Sigma_{m-i}\right)\right) \stackrel{\text { transfer }}{\longrightarrow} R^{0}\left(X \times B \Sigma_{m}\right)\right],
$$

where these are transfer maps associated to the subgroups $\Sigma_{i} \times \Sigma_{m-i} \subset \Sigma_{m}$. Thus $J \subseteq R^{0}\left(X \times B \Sigma_{m}\right)$ is an ideal. We write $\bar{P}_{m}$ for the composite map

$$
R^{0}(X) \stackrel{P_{m}}{\longrightarrow} R^{0}\left(X \times B \Sigma_{m}\right) \rightarrow R^{0}\left(X \times B \Sigma_{m}\right) / J .
$$

Proposition 3.15. The map $\bar{P}_{m}$ is a ring homomorphism.

Let $i: X \rightarrow X \times B \Sigma_{m}$ denote the map induced by inclusion of a basepoint in $B \Sigma_{m}$.

Proposition 3.16. The composite map

$$
R^{0}(X) \stackrel{P_{m}}{\longrightarrow} R^{0}\left(X \times B \Sigma_{m}\right) \stackrel{i^{*}}{\longrightarrow} R^{0}(X)
$$

sends $x \mapsto x^{m}$. 


\subsection{Relative power operations}

There is a "relative" version of the power operation, which we will need in $\S 12$. Let $(X, A)$ be a CW-pair of spaces, let $D_{m}(X, A) \subseteq X^{\times m}$ denote the space which is the union of the subspaces of the form $X^{i} \times A \times X^{m-i-1}$, and consider the diagram

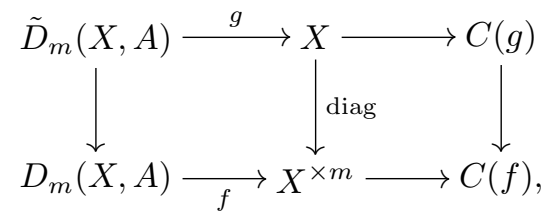

where $\tilde{D}_{m}(X, A)$ is the homotopy pullback of $f$ along the diagonal inclusion, and $C(f)$ and $C(g)$ are homotopy cofibers. The group $\Sigma_{m}$ acts on every space in this diagram. We define a pointed space

$$
B_{m}(X, A) \stackrel{\text { def }}{=} C(g)_{h \Sigma_{m}}^{\wedge m}
$$

it comes with a map $B_{m}(X, A) \rightarrow C(f)_{h \Sigma_{m}}^{\wedge m} \approx(X / A)_{h \Sigma_{m}}^{\wedge m}$. Note that $B_{m}(X, \varnothing) \approx$ $\left(X \times B \Sigma_{m}\right)_{+}$. Given such a pair $(X, A)$, we define

$$
P_{m}: \tilde{R}^{0}(X / A) \rightarrow \tilde{R}^{0} B_{m}(X, A)
$$

so that an $E$-module map $x: E \wedge \Sigma^{\infty} X / A \rightarrow R$ is sent to the composite

$$
E \wedge \Sigma^{\infty} B_{m}(X, A) \rightarrow E \wedge \Sigma^{\infty}(X / A)_{h \Sigma_{m}}^{\wedge m} \approx \mathbb{P}_{m}\left(E \wedge \Sigma^{\infty}(X / A)\right) \stackrel{\mathbb{P}_{m}(x)}{\longrightarrow} \mathbb{P}_{m} R \rightarrow R .
$$

The diagram

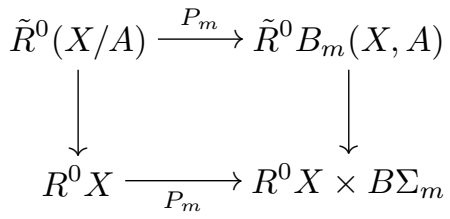

commutes.

We are mainly interested in pairs of the form $(D(V), S(V))$, where $V \rightarrow X$ is a real vector bundle. In this case, we see that the relative power operation amounts to a map

$$
P_{m}: \tilde{R}^{0} X^{V} \rightarrow \tilde{R}^{0}\left(X \times B \Sigma_{m}\right)^{V \otimes \rho_{m}}
$$

where $\rho_{m} \rightarrow B \Sigma_{m}$ is the real vector bundle associated to the real permutation representation, $V \otimes \rho_{m}$ is the external tensor product bundle, and the spaces are Thom spaces.

\subsection{Power operations in non-zero degree}

The definition of $\$ 3.9$ extends to arbitrary degree, as follows. Given $q \in \mathbb{Z}$, there is a function

$$
P_{m}: R^{q} X \rightarrow R^{q}\left(X_{+} \wedge B \Sigma_{m}^{-q \bar{\rho}_{m}}\right)
$$

where $\bar{\rho}_{m}$ denotes the real permutation representation; if $q>0$, then $-q \bar{\rho}_{m}$ is a virtual bundle, and thus the target of $P_{m}$ is really $R^{q}\left(\Sigma_{+}^{\infty} X \wedge B \Sigma_{m}^{-q \bar{\rho}_{m}}\right)$. The function $P_{m}$ is 
defined so that an $E$-module map $x: E \wedge \Sigma_{+}^{\infty} X \wedge S^{-q} \rightarrow R$ is sent to the composite

$$
\begin{aligned}
E \wedge \Sigma_{+}^{\infty} X \wedge B \Sigma_{m}^{-q \bar{\rho}_{m}} & \wedge S^{-q} \approx E \wedge \Sigma_{+}^{\infty} X \wedge B \Sigma_{m}^{-q \rho_{m}} \rightarrow \\
E & \wedge\left(\Sigma_{+}^{\infty} X \wedge S^{-q}\right)_{h \Sigma_{m}} \approx \mathbb{P}_{m}\left(E \wedge \Sigma_{+}^{\infty} X \wedge S^{-q}\right) \rightarrow \mathbb{P}_{m} R \rightarrow R .
\end{aligned}
$$

Let $X$ be a pointed space. If $m>0$, then the operator $P_{m}$ defined above restricts to a function

$$
P_{m}^{\prime}: \tilde{R}^{q} X \rightarrow \tilde{R}^{q}\left(X \wedge B \Sigma_{m}^{-q \bar{\rho}_{m}}\right) .
$$

Proposition 3.17. Let $X$ be a pointed space, and $q \in \mathbb{Z}$. The diagram

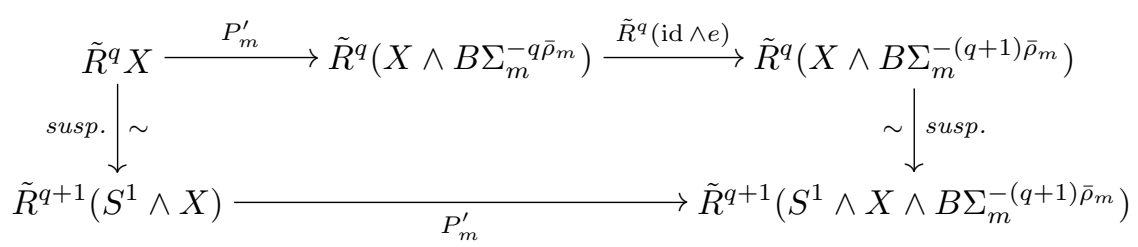

commutes, where the vertical maps are the suspension isomorphisms, and the map $e: B \Sigma_{m}^{-(q+1) \bar{\rho}_{m}} \rightarrow B \Sigma_{m}^{-q \bar{\rho}_{m}}$ is the map of Thom spectra induced by the inclusion $0 \subset$ $\rho_{m}$ of vector bundles.

Proof. A straightforward calculation, using the definitions.

The following corollary will be crucial for our treatment of gradings in $\S 6$. It relates the action of power operations on $R^{q} S^{q}$ with the action of power operations on $R^{0}(*)$.

Corollary 3.18. For all $q \geqslant 0$, the diagram

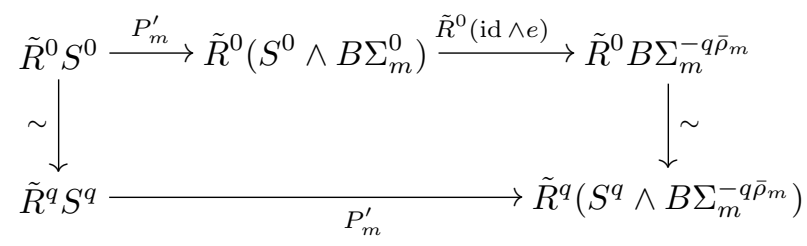

commutes, where $e: B \Sigma_{m}^{-q \bar{\rho}_{m}} \rightarrow B \Sigma_{m}^{0}$.

\section{Approximation functors}

In this section, we are going to produce a monad $\mathbb{T}$ on the category $\operatorname{Mod}_{E_{*}}$, called the algebraic approximation functor, and thus a category $\mathrm{Alg}_{\mathbb{T}}^{*}$ of algebras for the monad $\mathbb{T}$. There will be dotted arrow

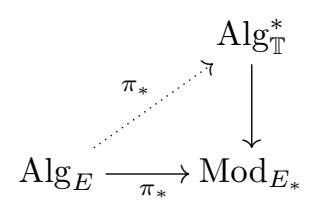

making the diagram commute up to natural isomorphism. Furthermore, the lift is be "nearly" optimal, in the sense that for a flat $E$-module spectrum $M$, the object 
$\pi_{*} L \mathbb{P} M$ is be "nearly" isomorphic to the free $\mathbb{T}$-algebra on $\pi_{*} M$. In the above sentence, "nearly" indicates that the isomorphism holds only after a suitable completion.

The category $\operatorname{Alg}_{\mathbb{T}}^{*}$ has a number of nice properties. Most notably, the forgetful functor to commutative $E_{*}$-algebras

$$
U: \operatorname{Alg}_{\mathbb{T}}^{*} \rightarrow \operatorname{Alg}_{E_{*}}
$$

admits both a left and a right adjoint, so that $U$ preserves both limits and colimits.

\subsection{Left Kan extension}

Recall that given functors $F: \mathcal{J} \rightarrow \mathcal{D}$ and $U: \mathcal{J} \rightarrow \mathcal{C}$, a left Kan extension of $F$ along $U$ is the initial example of a pair $(E, \delta)$, where $E: \mathcal{C} \rightarrow \mathcal{D}$ is a functor and $\delta: F \rightarrow E U$ is natural transformation of functors $\mathcal{J} \rightarrow \mathcal{D}$. We denote the left Kan extension (if it exists) by colim ${ }^{U} F$, and we write $\beta: F \rightarrow\left(\operatorname{colim}^{U} F\right) U$ for the canonical natural transformation. The universal property of the left Kan extension is equivalent to the following: if $G: \mathcal{C} \rightarrow \mathcal{D}$ is any functor, then there is a one-to-one correspondence between

(natural transformations $F \rightarrow G U) \Longleftrightarrow$ (natural transformations $\operatorname{colim}^{U} F \rightarrow G$ ).

Note that if $U$ is fully faithful, then $\beta: F \rightarrow\left(\operatorname{colim}^{U} F\right) U$ is a natural isomorphism.

Given essentially small J J choose a set of objects $S$ of $\mathcal{J}$ which spans its isomorphism classes. For $q \in \mathbb{Z}$, define a functor $B_{q}=B_{q}^{F, U}: \mathcal{C} \rightarrow \mathcal{D}$ by

$$
B_{q}(X) \stackrel{\text { def }}{=} \coprod_{\substack{I_{0} \rightarrow \cdots \rightarrow I_{q} \in \mathcal{J} \\ U I_{q} \rightarrow X \in \mathcal{C}}} F\left(I_{0}\right),
$$

where the direct sum is taken over all diagrams $I_{0} \rightarrow \cdots \rightarrow I_{q}$ in $\mathcal{J}$ where the objects are in $S$, together with all morphisms $U I_{q} \rightarrow X$ in $\mathcal{C}$. Then the left Kan extension is the coequalizer of the evident pair of arrows

$$
B_{1}(X) \rightrightarrows B_{0}(X) \rightarrow\left(\operatorname{colim}^{U} F\right)(X)
$$

Now suppose that we are additionally given a functor $V: \mathcal{C} \rightarrow \mathcal{C}^{\prime}$. Then we can form the left Kan extension of $F$ along $V U: \mathcal{J} \rightarrow \mathcal{C}^{\prime}$, and the canonical transformation $F \rightarrow\left(\operatorname{colim}^{V U} F\right) V U$ corresponds (according to the universal property of colim ${ }^{U}$ ) to a natural transformation $\gamma: \operatorname{colim}^{U} F \rightarrow\left(\operatorname{colim}^{V U} F\right) V$.

Lemma 4.1. Given a diagram of categories and functors

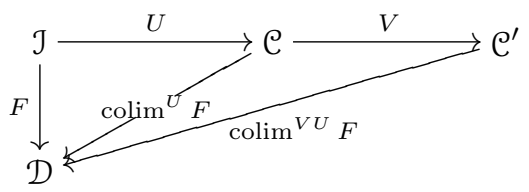

with $\mathcal{J}$ essentially small, if for all objects $I$ in $\mathcal{J}$ and $X$ in $\mathcal{C}$ the map $\mathrm{C}(U I, X) \rightarrow$ $\mathrm{C}^{\prime}(V U I, V X)$ is a bijection, then the transformation $\gamma: \operatorname{colim}^{U} F \rightarrow\left(\operatorname{colim}^{V U} F\right) V$ is an isomorphism.

Proof. Using the coequalizer (1) above, it suffices to check that the evident maps $B_{q}^{F, U}(X) \rightarrow B_{q}^{F, V U}(V X)$ are isomorphims, which is immediate from the hypothesis. 


\subsection{Construction of $\mathbb{T}_{m}$ and $\mathbb{T}$}

In this section, we construct the functors $\mathbb{T}_{m}: \operatorname{Mod}_{E_{*}} \rightarrow \operatorname{Mod}_{E_{*}}$, called algebraic approximation functors, We will define $\mathbb{T}(M) \stackrel{\text { def }}{=} \bigoplus_{m \geqslant 0} \mathbb{T}_{m}(M)$.

The idea is to define $\mathbb{T}_{m}$ first on finitely generated free $E_{*}$-modules using the equivalence of categories $\pi_{*}: h \operatorname{Mod}_{E}^{\mathrm{ff}} \rightarrow \operatorname{Mod}_{E_{*}}^{\mathrm{ff}}$. Thus, for a finite free $E_{*}$-module $M_{*}$, we should set $\mathbb{T}_{m}\left(M_{*}\right)=\pi_{*} L \mathbb{P}_{m}(M)$, where $M$ is an $E$-module such that $\pi_{*} M \approx M_{*}$. Then we extend $\mathbb{T}_{m}$ to all $E_{*}$-modules via left Kan extension.

We will refer to the following diagram of functors

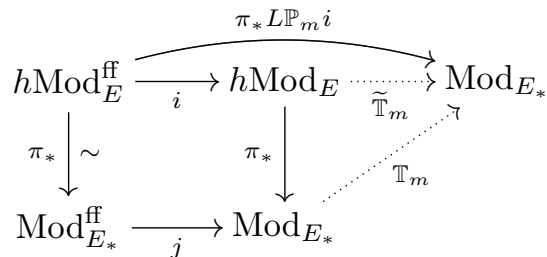

in which the left-hand square commutes (on the nose), the functors $i$ and $j$ are inclusions of full subcategories, and the vertical arrow on the left is an equivalence of categories.

We define the functor $\mathbb{T}_{m}: \operatorname{Mod}_{E_{*}} \rightarrow \operatorname{Mod}_{E_{*}}$ be the left Kan extension of the functor $\pi_{*} L \mathbb{P}_{m} i: h \operatorname{Mod}_{E}^{\mathrm{ff}} \rightarrow \operatorname{Mod}_{E_{*}}$ along the functor $\pi_{*} i=j \pi_{*}: h \operatorname{Mod}_{E}^{\mathrm{ff}} \rightarrow \operatorname{Mod}_{E_{*}}$; this exists because $h \operatorname{Mod}_{E}^{\mathrm{ff}}$ is essentially small.

The functor $\pi_{*} i$ is fully faithful, and so the tautological transformation

$$
\beta: \pi_{*} L \mathbb{P}_{m} i \rightarrow \mathbb{T}_{m} \pi_{*} i
$$

is an isomorphism.

Lemma 4.2. The natural map $\kappa: \mathbb{T}_{m} \rightarrow \operatorname{colim}^{j} \mathbb{T}_{m} j$, which is adjoint to the identity $\mathrm{id}: \mathbb{T}_{m} j \rightarrow \mathbb{T}_{m} j$, is an isomorphism.

Proof. Since $\beta$ is an isomorphism and $\pi_{*} i=j \pi_{*}$, we have natural isomorphisms

$$
\mathbb{T}_{m}=\operatorname{colim}^{\pi_{*} i} \pi_{*} L \mathbb{P} i \approx \operatorname{colim}^{j \pi_{*}} \mathbb{T}_{m} j \pi_{*} .
$$

Since $\pi_{*}: h \operatorname{Mod}_{E}^{\mathrm{ff}} \rightarrow \operatorname{Mod}_{E_{*}}^{\mathrm{ff}}$ is an equivalence of categories, we see that

$$
\operatorname{colim}^{j \pi_{*}} \mathbb{T}_{m} j \pi_{*} \approx \operatorname{colim}^{j} \mathbb{T}_{m} j
$$

\subsection{Construction of the approximation map}

Let $\widetilde{\mathbb{T}}_{m}: h \operatorname{Mod}_{E} \rightarrow \operatorname{Mod}_{E_{*}}$ be the left Kan extension of the functor

$$
\pi_{*} L \mathbb{P}_{m} i: h \operatorname{Mod}_{E}^{\mathrm{ff}} \rightarrow \operatorname{Mod}_{E_{*}}
$$

along the inclusion $i: h \operatorname{Mod}_{E}^{\mathrm{ff}} \rightarrow h \operatorname{Mod}_{E}$. There are natural isomorphisms

$$
\mathbb{T}_{m} \pi_{*} i \stackrel{\beta}{\longleftarrow} \pi_{*} L \mathbb{P}_{m} i \stackrel{\text { id }}{\longrightarrow} \pi_{*} L \mathbb{P}_{m} i,
$$

where $\beta$ is the tautological natural transformation for $\mathbb{T}_{m}$, which are associated (since $\widetilde{\mathbb{T}}_{m}$ is a left Kan extension along $i$ ), to natural transformations

$$
\mathbb{T}_{m} \pi_{*} \stackrel{\gamma}{\longleftarrow} \widetilde{\mathbb{T}}_{m} \stackrel{\tilde{\alpha}}{\longrightarrow} \pi_{*} L \mathbb{P}_{m}
$$

of functors $h \operatorname{Mod}_{E} \rightarrow \operatorname{Mod}_{E_{*}}$. 
Lemma 4.3. The map $\gamma$ is a natural isomorphism.

Proof. We are precisely in the setup of (4.1), using that

$$
\pi_{*}: h \operatorname{Mod}_{E}(F, M) \rightarrow \operatorname{Mod}_{E_{*}}\left(\pi_{*} F, \pi_{*} M\right)
$$

is an isomorphism for all $F$ in $h \operatorname{Mod}_{E}^{\mathrm{ff}}$.

The natural transformation

$$
\alpha_{m}: \mathbb{T}_{m}\left(\pi_{*} M\right) \rightarrow \pi_{*}\left(L \mathbb{P}_{m}(M)\right)
$$

is defined by $\alpha_{m}=\tilde{\alpha} \gamma^{-1}$.

Proposition 4.4. When $\pi_{*} M$ is a finite free $E_{*}$-module, the map $\alpha_{m}: \mathbb{T}_{m}\left(\pi_{*} M\right) \rightarrow$ $\pi_{*} L \mathbb{P}_{m}(M)$ is an isomorphism.

The natural transformation

$$
\alpha: \mathbb{T}\left(\pi_{*} M\right) \rightarrow \pi_{*} L \mathbb{P}(M)
$$

is defined by

$$
\bigoplus_{m} \mathbb{T}_{m}\left(\pi_{*} M\right) \stackrel{\alpha_{m}}{\longrightarrow} \bigoplus_{m} \pi_{*} L \mathbb{P}_{m} M \rightarrow \pi_{*} L\left(\bigvee_{m} L \mathbb{P}_{m} M\right) \approx \pi_{*} L \mathbb{P} M
$$

Note that the analogue to (4.4) does not hold for $\alpha$.

\section{4. $\quad \mathbb{T}$ is a monad}

Proposition 4.5. The functor $\mathbb{T}: \operatorname{Mod}_{E_{*}} \rightarrow \operatorname{Mod}_{E_{*}}$ admits the structure of a monad, compatibly with the monad structure of $L \mathbb{P}$, in the sense that the diagrams
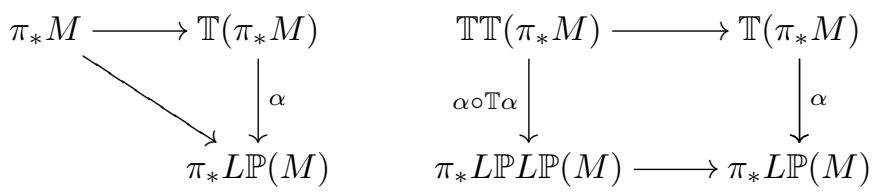

commute, with the unlabeled maps being the ones describing the monad structure.

Proof. The structure maps $I \rightarrow \mathbb{T}$ and $\mathbb{T} \mathbb{T} \rightarrow \mathbb{T}$ of the monad are defined on finite free modules using the maps

$$
\pi_{*} X \rightarrow \pi_{*} L \mathbb{P} X \quad \text { and } \quad \pi_{*} L \mathbb{P} L \mathbb{P} X \rightarrow \pi_{*} L \mathbb{P} X
$$

for $X \in \operatorname{Mod}_{E}^{\mathrm{ff}}$, together with the equivalence $L \mathbb{P P} \rightarrow L \mathbb{P} L \mathbb{P}$ as in (3.8).

\subsection{Colimits}

Proposition 4.6. The functors $\mathbb{T}_{m}$ commute with filtered colimits and reflexive coequalizers. 
Proof. Observe that for $q=0,1$, the functor

$$
M \mapsto B_{q}(M)=\bigoplus_{\substack{F_{0} \rightarrow \ldots \rightarrow F_{q} \in h \operatorname{Mod}_{E}^{\mathrm{ff}} \\ \pi_{*} F_{q} \rightarrow M \in \operatorname{Mod}_{E_{*}}}} \pi_{*} L \mathbb{P}_{m} F_{0}
$$

from $\operatorname{Mod}_{E_{*}} \rightarrow \operatorname{Mod}_{E_{*}}$ preserves filtered colimits, since the objects $\pi_{*} F_{q}$ of $\operatorname{Mod}_{E_{*}}$ are small, in the sense that $\operatorname{hom}_{\operatorname{Mod}_{E_{*}}}\left(\pi_{*} F_{q},-\right)$ preserves filtered colimits. The filtered colimit part of the result follows using (1).

The functors $M \mapsto B_{q}(M)$ also reflexive coequalizers, since the objects $\pi_{*} F_{q}$ of $\operatorname{Mod}_{E_{*}}$ are projective, in the sense that $\operatorname{hom}_{\operatorname{Mod}_{E *}}\left(\pi_{*} F_{q},-\right)$ carries epimorphisms to surjections. Thus the reflexive coequalizer part of the result follows using (1).

\subsection{Tensor products}

Let $k \geqslant 0$, and $M_{1}, \ldots, M_{k} \in \operatorname{Mod}_{E_{*}}$. We define a natural map

$$
\gamma_{k}: \mathbb{T}\left(M_{1}\right) \otimes \cdots \otimes \mathbb{T}\left(M_{k}\right) \rightarrow \mathbb{T}\left(M_{1} \oplus \cdots \oplus M_{k}\right)
$$

as follows. As in the proof of (4.6), let

$$
B_{q}(M)=\bigoplus_{\substack{F_{0} \rightarrow \cdots \rightarrow F_{q} \in \operatorname{Mod}_{E_{*}}^{\mathrm{ff}} \\ F_{q} \rightarrow M \in \operatorname{Mod}_{E_{*}}}} \bigoplus_{m} \pi_{*} L \mathbb{P}_{m} F_{0},
$$

so that $\mathbb{T}(M) \approx H_{0} B(M)$. We have maps

$$
B_{q_{1}}\left(M_{1}\right) \otimes \cdots \otimes B_{q_{k}}\left(M_{k}\right) \stackrel{s}{\rightarrow} B_{q}\left(M_{1}\right) \otimes \cdots \otimes B_{q}\left(M_{k}\right) \stackrel{t}{\rightarrow} B_{q}\left(M_{1} \oplus \cdots \oplus M_{k}\right)
$$

for $q=\sum q_{i}$, where $s$ is the Eilenberg-Mac Lane shuffle map, and $t$ is the map constructed in the evident way from the "exponential isomorphism" maps

$$
\pi_{*} L \mathbb{P}_{m_{1}} F_{1} \otimes \cdots \otimes \pi_{*} L \mathbb{P}_{m_{k}} F_{k} \rightarrow \pi_{*} L \mathbb{P}_{m_{1}+\cdots+m_{k}}\left(F_{1} \vee \cdots \vee F_{k}\right) .
$$

These are maps of chain complexes, and taking the 0th homology group gives the desired map $\gamma_{k}$.

For the following, we will need to make use of comma categories. Given a category $\mathcal{C}$ and an object $X$ of $\mathcal{C}$, the comma category $\mathcal{C} / X$ is the category whose objects are pairs $(Y, f: Y \rightarrow X)$ where $Y$ is an objects of $\mathcal{C}$, and morphisms $(Y, f) \rightarrow\left(Y^{\prime}, f^{\prime}\right)$ are maps $g: Y \rightarrow Y^{\prime}$ such that $f^{\prime} g=f$.

Proposition 4.7. The map $\gamma_{k}$ is an isomorphism.

Proof. It is standard that $H_{0}(s)$ is an isomorphism, so it suffices to show that $H_{0}(t)$ is an isomorphism. Consider the comma categories

$$
\mathcal{C}=\left(\prod \pi_{*}:\left(h \operatorname{Mod}_{E}^{\mathrm{ff}}\right)^{k} \rightarrow\left(\operatorname{Mod}_{E_{*}}^{k}\right)\right) /\left(M_{1}, \ldots, M_{k}\right)
$$

and

$$
\mathcal{D}=\left(\pi_{*}: h \operatorname{Mod}_{E}^{\mathrm{ff}} \rightarrow \operatorname{Mod}_{E_{*}}\right) /\left(M_{1} \oplus \cdots \oplus M_{k}\right),
$$

and let $\rho: \mathcal{C} \rightarrow \mathcal{D}$ be the functor sending

$$
\left(F_{i}, f_{i}: \pi_{*} F_{i} \rightarrow M_{i}\right)_{i=1, \ldots, k} \quad \text { to } \quad\left(\vee F_{i},\left(f_{i}\right): \pi_{*}\left(\vee F_{i}\right) \rightarrow \oplus M_{i}\right) .
$$

Let $R: \mathcal{C} \rightarrow \operatorname{Mod}_{E_{*}}$ be the functor sending $\left(F_{i}, f_{i}\right)$ to $\bigoplus \pi_{*} L \mathbb{P}_{m_{1}} F_{1} \otimes \cdots \pi_{*} L \mathbb{P}_{m_{k}} F_{k}$, let $S: \mathcal{D} \rightarrow \operatorname{Mod}_{E_{*}}$ be the functor sending $(F, f)$ to $\bigoplus \pi_{*} L \mathbb{P}_{m} F$. Let $h: R \rightarrow S \rho$ be 
the evident natural isomorphism. It is clear that $H_{0}(t)$ is isomorphic to the map

$$
\operatorname{colim}^{\mathcal{C}} R \approx \operatorname{colim}^{\mathcal{C}} S \rho \stackrel{\eta}{\rightarrow} \operatorname{colim}^{\mathcal{D}} S,
$$

and the result follows from the observation that $\rho$ admits a left adjoint and therefore $\eta$ is an isomorphism.

As a consequence, $\mathbb{T}(M)$ has a natural structure of a commutative ring, with product defined by $\delta_{2}: \mathbb{T}(M) \otimes \mathbb{T}(M) \stackrel{\gamma_{2}}{\longrightarrow} \mathbb{T}(M \oplus M) \stackrel{\mathbb{T}\left(\left(\operatorname{id}_{M}, \operatorname{id}_{M}\right)\right)}{\longrightarrow} \mathbb{T}(M)$.

The naturality of the construction of $\gamma_{k}$ shows the following.

Corollary 4.8. The natural isomorphisms $\gamma_{k}$ give $\mathbb{T}$ the structure of a symmetric monoidal functor $\left(\operatorname{Mod}_{E_{*}}, 0, \oplus\right) \rightarrow\left(\operatorname{Mod}_{E_{*}}, E_{*}, \otimes\right)$. Furthermore, the monad structure maps $\eta: I \rightarrow \mathbb{T}$ and $\mu: \mathbb{T} \mathbb{T} \rightarrow \mathbb{T}$ are maps of monoidal functors.

Proof. Reduce to the case of free modules.

\subsection{Completed approximation functor}

We construct completed approximation functors, which are better approximations to the homotopy of the $K(n)$-localization of a free $E$-algebra, but which are less convenient to deal with algebraically. Thus we define $\widehat{\mathbb{T}}(M) \stackrel{\text { def }}{=} L_{0} \mathbb{T}(M)$ where $L_{0}$ is the functor of $(3.3)$, and we let $\widehat{\alpha}: \widehat{\mathbb{T}}\left(\pi_{*} M\right) \rightarrow \pi_{*} L \mathbb{P}(M)$ be the unique factorization of $\alpha$ through $\widehat{\mathbb{T}}(M)$.

Proposition 4.9. If $M$ is a flat E-module, then the maps $\widehat{\mathbb{T}}\left(\pi_{*} M\right) \rightarrow\left[\mathbb{T}\left(\pi_{*} M\right)\right]_{\mathfrak{m}}^{\wedge}$ and $\widehat{\alpha}: \widehat{\mathbb{T}}\left(\pi_{*} M\right) \rightarrow \pi_{*} L \mathbb{P}(M)$ are isomorphisms.

Proof. Since $M$ is a flat $E$-module, $\mathbb{T} \pi_{*} M$ is a flat $E_{*}$ module (since $\mathbb{T}$ commutes with filtered colimits (4.6)), and the first isomorphism follows using (3.2).

Since $M$ is a flat module, then $M \approx \operatorname{hocolim}_{J} M_{j}$ for some filtered topological category $J$, where the $M_{\alpha}$ are finite free (3.7). Let $\mathbb{N}$ denote the category whose objects are natural numbers, and which has no non-identity maps. Consider the $E$ module

$$
N=\operatorname{hocolim}_{(i, j) \in \mathbb{N} \times J} L \mathbb{P}_{i} M_{j} .
$$

Since each $L \mathbb{P}_{i} M_{j}$ is finite free, the $E$-module $N$ is flat, and thus the map $\beta: \pi_{*} N \rightarrow$ $\pi_{*} L N$ factors through an isomorphism $L_{0} \pi_{*} N \rightarrow \pi_{*} L N$ by (3.4). It is then straightforward to check that $\beta$ is isomorphic to the approximation map

$$
\operatorname{colim} \mathbb{T}_{i} \pi_{*} M_{j} \approx \mathbb{T} \pi_{*} M \stackrel{\alpha}{\rightarrow} \pi_{*} L \operatorname{hocolim} L \mathbb{P}_{i} M_{j} \approx \pi_{*} L \mathbb{P} M .
$$

Note that in the above, we have assumed that $L$ is a continuous functor. We may in fact do this, for instance using the description of $L$ given in $(3.1)(3)$.

\subsection{T-algebras}

As we have observed above, the functor $\mathbb{T}: \operatorname{Mod}_{E_{*}} \rightarrow \operatorname{Mod}_{E_{*}}$ is a monad. Let $\operatorname{Alg}_{\mathbb{T}}^{*}$ denote the category of $\mathbb{T}$-algebras. Every object of $\mathrm{Alg}_{\mathbb{T}}^{*}$ is a graded commutative $E_{*^{-}}$ algebra, and so there is a forgetful functor $U: \operatorname{Alg}_{\mathbb{T}}^{*} \rightarrow \operatorname{Alg}_{E_{*}}$. (Note that the image of $U$ is contained inside the strictly graded commutative $E_{*}$-algebras, by (3.14).) 
Corollary 4.10. The forgetful functor $U: \operatorname{Alg}_{\mathbb{T}}^{*} \rightarrow \operatorname{Alg}_{E_{*}}$ commutes with colimits.

Proof. It suffices to show that $U$ commutes with filtered colimits, reflexive coequalizers, and finite coproducts. That it commutes with the first two types of colimit is immediate from (4.6).

\subsection{Power operations, revisited}

Let $B$ be a $\mathbb{T}$-algebra. We obtain functions

$$
P_{m}: B_{0} \rightarrow \operatorname{hom}_{\operatorname{Mod}_{E_{*}}}\left(\mathbb{T}_{m} E_{*}, B\right)
$$

which are defined by sending an $E_{*}$-module homomorphism $b: E_{*} \rightarrow B$ to the composite

$$
\mathbb{T}_{m} E_{*} \rightarrow \mathbb{T}_{m} B \rightarrow B
$$

We call the map $P_{m}$ a power operation. If $R$ is a commutative $E$-algebra spectrum, then we see that this operation is identified with the operation $P_{m}: R^{0}(*) \rightarrow R^{0} B \Sigma_{m}$ defined earlier, via the natural isomorphism $R^{0} B \Sigma_{m} \approx \operatorname{hom}_{\operatorname{Mod}_{E_{*}}}\left(E_{0}^{\wedge} B \Sigma_{m}, R_{0}\right)$.

Let $f: E_{*}[x] \rightarrow \mathbb{T} E_{*}$ be the map from the free commutative $E_{*}$-algebra on one generator which sends $x$ to the tautological generator of $\mathbb{T} E_{*}$ as a $\mathbb{T}$-algebra. Let $f_{m}: E_{*} \rightarrow \mathbb{T}_{m} E_{*}$ be the restriction of $f$ to the degree $m$ part of $E_{*}[x]$. In terms of topology, $f_{m}$ is the map $i_{*}: E_{*} \approx E_{*}^{\wedge}(*) \rightarrow E_{*}^{\wedge} B \Sigma_{m}$ induced by basepoint inclusion.

Lemma 4.11. The composite map

$$
B_{0} \stackrel{P_{m}}{\longrightarrow} \operatorname{hom}_{\operatorname{Mod}_{E_{*}}}\left(\mathbb{T}_{m} E_{*}, B\right) \rightarrow \operatorname{hom}_{\operatorname{Mod}_{E_{*}}}\left(E_{*}, B_{0}\right) \approx B_{0}
$$

is the map which sends $x \mapsto x^{m}$

Proof. Use (3.16).

\subsection{Plethories and plethyistic functors}

Let $\mathcal{C}$ be an abelian tensor category with tensor product $\otimes$ and unit object $\mathbb{k}$, and let $\mathcal{A}$ denote the category of commutative monoid objects in $\mathcal{C}$ with respect to the tensor product. Say that a functor $U: \mathcal{D} \rightarrow \mathcal{A}$ is plethyistic if

(1) $U$ reflects isomorphisms (i.e., $U(f)$ iso implies $f$ iso), and

(2) $U$ admits both a left adjoint $F$ and a right adjoint $G$.

It is a consequence of this definition (using Beck's theorem [Mac71]) that if $M=U F$ and $C=U G$ are the monad and comonad associated to these adjoint pairs, then $\mathcal{D}$ is equivalent to the categories of $M$-algebras and $C$-coalgebras.

The basic example of a plethyistic functor occurs when $\mathcal{C}=\operatorname{Mod}_{R}$ for some commutative ring $R$. Then $U: \mathcal{D} \rightarrow \operatorname{Alg}_{R}$ amounts to what Borger and Wieland call a plethory [BW05]. (More precisely, Borger and Wieland define a plethory to be a commutative ring $P$ equipped with some additional structure; they extract a plethyistic functor from this data, in such a way that $P=M(R)$. Furthermore, they show [BW05, Thm. 4.9] that a plethyistic functor $U: \mathcal{D} \rightarrow \operatorname{Alg}_{R}$ determines a plethory in their sense.)

Proposition 4.12. The functor $U: \operatorname{Alg}_{\mathbb{T}}^{*} \rightarrow \operatorname{Alg}_{E_{*}}$ is plethyistic. 
Proof. It is clear from the definitions that $U$ reflects isomorphisms, and preserves limits. It clear from (4.6) and (4.7) that $U$ preserves colimits.

Next, we construct a left adjoint $F$ to $U$. Let $\mathcal{C}$ denote the full subcategory of $\operatorname{Alg}_{E_{*}}$ consisting of $E_{*}$-algebras $A_{*}$ for which there exists an object $F^{\prime}\left(A_{*}\right) \in \operatorname{Alg}_{\mathbb{T}}^{*}$ and a natural isomorphism $\operatorname{Alg}_{\mathbb{T}}^{*}\left(F^{\prime}\left(A_{*}\right), B_{*}\right) \approx \operatorname{Alg}_{E_{*}}\left(A_{*}, U\left(B_{*}\right)\right)$; the left adjoint $F$ exists if $\mathcal{C}=\operatorname{Alg}_{E_{*}}$. Since $U$ preserves limits, $\mathcal{C}$ is closed under colimits in $\operatorname{Alg}_{E_{*}}$, and thus it suffices to show that $\operatorname{Sym}^{*}\left(E_{0}\right)$ and $\operatorname{Sym}^{*}\left(\omega^{1 / 2}\right)$ are in $\mathcal{C}$. But it is straightforward to check that we can take $F^{\prime}\left(\operatorname{Sym}^{*}\left(E_{0}\right)\right) \approx \mathbb{T}\left(E_{0}\right)$ and $F^{\prime}\left(\operatorname{Sym}^{*}\left(\omega^{1 / 2}\right)\right) \approx \mathbb{T}\left(\omega^{1 / 2}\right)$.

It follows by Beck's theorem that $U$ is monadic, since $U$ preserves colimits (4.10).

Next, we construct a right adjoint $G: \operatorname{Alg}_{E_{*}} \rightarrow \operatorname{Alg}_{\mathbb{T}}^{*}$ to $U$. For a $\mathbb{T}$-algebra $A$, consider the functor $X:\left(\operatorname{Alg}_{\mathbb{T}}^{*}\right)^{\text {op }} \rightarrow$ Set defined by

$$
X(B)=\operatorname{Hom}_{\mathrm{Alg}_{E_{*}}}(U B, A) .
$$

Since $U$ preserves colimits, $X$ carries colimits to limits. The category $\operatorname{Alg}_{\mathbb{T}}^{*}$ is locally presentable, and so $X$ must be representable by an object $G A$ in $\operatorname{Alg}_{\mathbb{T}}^{*}$.

\subsection{Weight decomposition}

Suppose that $U: \mathcal{D} \rightarrow \mathcal{C}$ is a plethyistic functor, and that the forgetful functor $\mathcal{A} \rightarrow$ $\mathcal{C}$ is monadic. Then the composite forgetful functor $\mathcal{D} \rightarrow \mathcal{A} \rightarrow \mathcal{C}$ is also monadic. We write $T: \mathcal{C} \rightarrow \mathcal{C}$ for this monad. For future reference, we note the following structure carried by $T$, namely natural maps of functors $\mathcal{C} \rightarrow \mathcal{C}$ :

$$
\begin{array}{ll}
\eta: M \rightarrow T(M), & \mu: T T(M) \rightarrow T(M), \\
\iota: \mathbb{k} \rightarrow T(M), & \delta: T(M) \otimes T(M) \rightarrow T(M),
\end{array}
$$

which define the monad and commutative ring structures on the functor $T$.

A weight decomposition of $T$ is a collection of functors $T_{k}: \mathcal{C} \rightarrow \mathcal{C}$ and a natural isomorphism $T \approx \bigoplus_{k \geqslant 0} T_{k}$ such that the dotted arrows exist in each of the following diagrams:
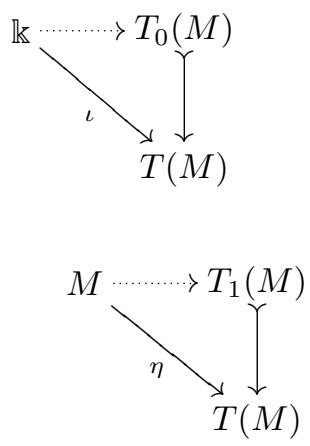
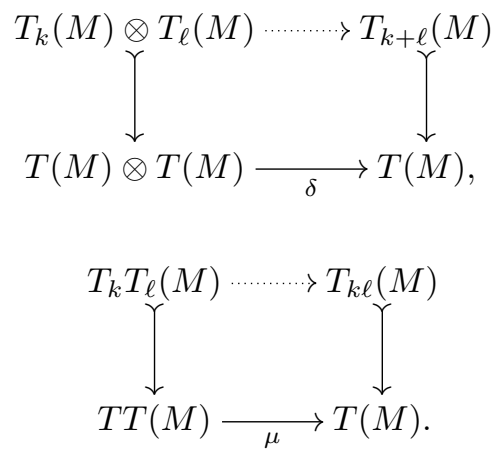

In each case, the dotted arrow is unique if it exists.

In the case of the plethyistic functor $\mathrm{Alg}_{\mathbb{T}}^{*} \rightarrow \mathrm{Alg}_{E_{*}}, T$ is precisely the algebraic approximation functor $\mathbb{T}$. It is clear that the standard splitting $\mathbb{T} \approx \bigoplus_{k \geqslant 0} \mathbb{T}_{k}$ is a weight decomposition for $\mathbb{T}$. 
THE CONGRUENCE CRITERION FOR POWER OPERATIONS IN MORAVA E-THEORY 349

\subsection{Suspension map}

There are natural "suspension maps"

$$
E_{q}: \Sigma^{q} L \mathbb{P}_{m}(X) \rightarrow L \mathbb{P}_{m}\left(\Sigma^{q} X\right)
$$

for $q \geqslant 0$. This gives rise to a map of $E_{*}$-modules

$$
E_{q}: \omega^{-q / 2} \otimes \mathbb{T}_{m}(M) \rightarrow \mathbb{T}_{m}\left(\omega^{-q / 2} \otimes M\right)
$$

which we will also call a suspension map.

We record some properties of the suspension map below. They all reduce to corresponding properties of the extended power functors.

Proposition 4.13. The diagram

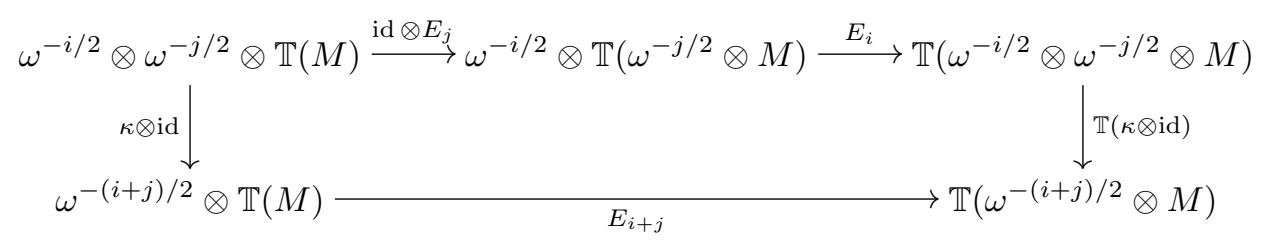

commutes in $\operatorname{Mod}_{E_{*}}$ for all $i, j \geqslant 0$ and all $M \in \operatorname{Mod}_{E_{*}}$.

Proposition 4.14. The diagrams

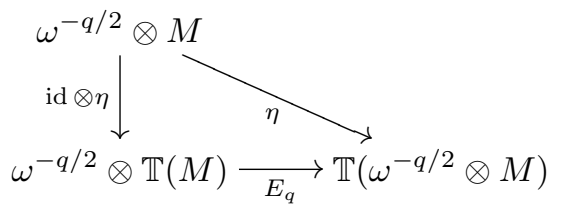

and

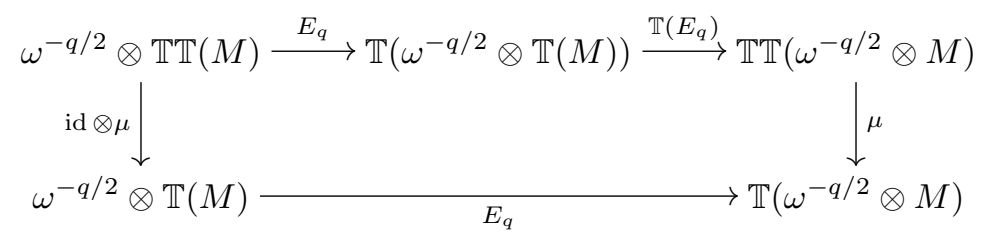

commute in $\operatorname{Mod}_{E_{*}}$ for all $q \geqslant 0$ and all $M \in \operatorname{Mod}_{E_{*}}$.

Define $\nu: \mathbb{T}(M \otimes N) \rightarrow \mathbb{T}(M) \otimes \mathbb{T}(N) \approx \mathbb{T}(M \oplus N)$ to be the unique $\mathbb{T}$-algebra map which extends $\eta \otimes \eta: M \otimes N \rightarrow \mathbb{T}(M) \otimes \mathbb{T}(N)$.

Proposition 4.15. The diagram

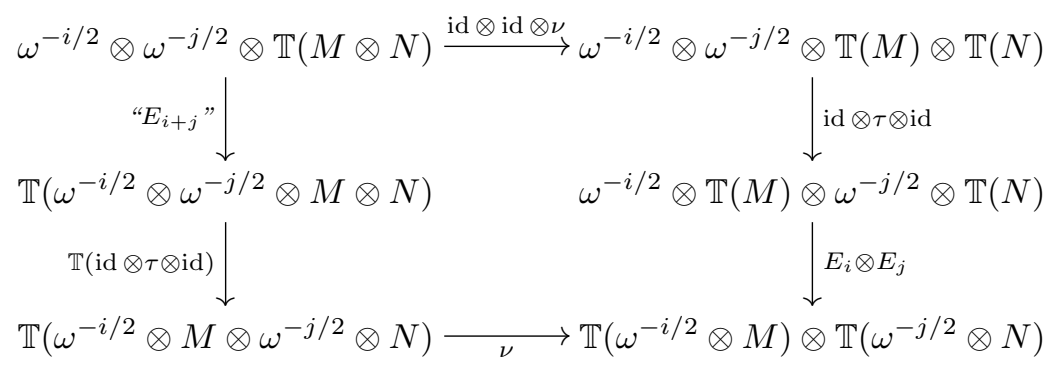


commutes in $\operatorname{Mod}_{E_{*}}$ for all $i, j \geqslant 0$ and all $M, N \in \operatorname{Mod}_{E_{*}}$, where " $E_{i+j}$ " means the map defined by composition along the top of the diagram in (4.13).

\section{Twisted bialgebras}

Let $R$ be a commutative ring. An algebra under $R$ is an associative ring $\Gamma$ together with a ring homomorphism $\eta: R \rightarrow \Gamma$. Note that the image of $\eta$ is not assumed to be central (in which case $\Gamma$ is an $R$-algebra).

An twisted cocommutative $R$-bialgebra (or bialgebra) is an algebra $\Gamma$ under $R$ which is equipped with certain additional structures which make the category of right $\Gamma$-modules into a symmetric monoidal category, in such a way that this symmetric monoidal product coincides with the tensor product over $R$ of the underlying $R$-modules. In the case when $R$ is central in $\Gamma$, then we have the conventional notion of a cocommutative $R$-bialgebra (like a cocommutative Hopf algebra, but without an antipode).

The original definition of bialgebra is due to Sweedler [Swe74]; it was modified by [Tak77], under the name of " $\times_{R}$-bialgebra". (Sweedler described a somewhat more general situation, in which the monoidal structure is not required to be symmetric.) This notion as well as various generalizations have been much studied in the literature on Hopf algebras and allied notions. It has entered topology in various guises. I first learned about the notion from [Voe03]; the motivic Steenrod algebra is naturally a twisted bialgebra over the motivic homology of the base scheme. Bialgebras can be thought of as a kind of "dual" version of the notion of Hopf algebroids (or more precisely, of "affine category schemes"), an observation we will make explicit below $(\S 5.5)$.

I have taken the terminology "twisted bialgebra" and some notation from [BW05].

\subsection{Bimodules, multimorphisms, and functors}

Let $R$ be a commutative ring, and suppose that $P$ and $Q$ be $R$-bimodules. We write

$$
\begin{aligned}
& P_{R} \otimes{ }_{R} Q \stackrel{\text { def }}{=} P \otimes Q /(p r \otimes q \sim p \otimes r q), \\
& P_{R} \otimes Q_{R} \stackrel{\text { def }}{=} P \otimes Q /(p r \otimes q \sim p \otimes q r) .
\end{aligned}
$$

Because $R$ is commutative, each of these admit three possible $R$-modules structures. For instance, $P_{R} \otimes Q_{R}$ admits the following three $R$-module structures (two on the left, one on the right):

$$
r \cdot{ }_{1}(p \otimes q)=r p \otimes q, \quad r \cdot{ }_{2}(p \otimes q)=p \otimes r q, \quad(p \otimes q) \cdot r=p r \otimes q=p \otimes q r .
$$

If $P$ and $Q_{1}, \ldots, Q_{K}$ are $R$-bimodules, then a $k$-multimorphism is a function $f: P \rightarrow\left(Q_{1}\right)_{R} \otimes \cdots \otimes\left(Q_{k}\right)_{R}$ which is a map of right $R$-modules, and which is a map of left $R$-modules for each of the $k$ left $R$-module structures on the target. Note that a 0-multimorphism is just a map $P \rightarrow R$ of right $R$-modules.

Given an $R$-bimodule $P$, let $H_{M}: \operatorname{Mod}_{R} \rightarrow \operatorname{Mod}_{R}$ denote the functor defined by $H_{P}(M)=\operatorname{hom}_{R}(P, M)$, where this means the set of homomorphisms with respect to the right $R$-module structure on $P$. Thus, $f \in H_{P}(M)$ is a additive map $f: P \rightarrow M$ 
such that $f(p r)=f(p) r$ for all $p \in P$ and $r \in R$. A bimodule map $b: P \rightarrow Q$ induces a natural transformation $\tilde{b}: H_{Q} \rightarrow H_{P}$, defined by

$$
\tilde{b}: H_{Q}(M) \rightarrow H_{P}(M), \quad f \mapsto(p \mapsto f(b(p)) .
$$

More generally, a $k$-multimorphism $b: P \rightarrow\left(Q_{1}\right)_{R} \otimes \cdots \otimes\left(Q_{k}\right)_{R}$ induces a natural transformations

$$
\begin{aligned}
\tilde{b}: H_{Q_{1}}\left(M_{1}\right)_{R} \otimes \cdots \otimes H_{Q_{k}}\left(M_{k}\right)_{R} & \rightarrow H_{P}\left(M_{1} \otimes_{R} \cdots \otimes_{R} M_{k}\right) \\
\left(f_{1} \otimes \cdots \otimes f_{k}\right) & \mapsto\left(p \mapsto \sum_{\alpha} f\left(q_{1}^{\alpha}\right) \otimes \cdots \otimes f\left(q_{k}^{\alpha}\right)\right),
\end{aligned}
$$

where $b(p)=\sum_{\alpha} q_{1}^{\alpha} \otimes \cdots \otimes q_{k}^{\alpha}$. In particular, a 0-multimorphism $b: P \rightarrow R$ induces

$$
\tilde{b}: R \rightarrow H_{P}(R), \quad r \mapsto(p \mapsto b(r p)) .
$$

Remark 5.1. The assignments $b \mapsto \tilde{b}$ described above satisfy a number of useful properties, relating to composition of functors and to the tensor product of $R$-modules; these can be safely left to the reader. The language of "multicategories" [Lei04, §2.1] can be used to encapsulate these properties: bimodules and multimorphisms form a multicategory $\mathcal{B}$, additive endofunctors $\operatorname{Mod}_{R} \rightarrow \operatorname{Mod}_{R}$ and multilinear natural transformations form a multicategory $\mathcal{F}$, and there is a morphism $H: \mathcal{B} \rightarrow \mathcal{F}$ of multicategories. Furthermore, $\mathcal{B}$ and $\mathcal{F}$ admit an additional "monoidal" structure (corresponding to bimodule tensor product and functor composition respectively), and $H$ is compatible with this monoidal structure (in the weak sense).

\subsection{Twisted commutative $R$-bialgebra}

Let $R$ be a commutative ring. An $R$-bialgebra (more precisely, a twisted cocommutative $R$-bialgebra is data $(\Gamma, \epsilon, \Delta, \eta, \mu)$, consisting of

(a) an associative ring $\Gamma$;

(b) a map $\eta: R \rightarrow \Gamma$ of rings;

(c) a map $\epsilon: \Gamma \rightarrow R$ of right $R$-modules such that $\epsilon(\eta(r))=r$ for all $r \in R$ and $\epsilon(x y)=\epsilon(\eta(\epsilon(x)) y)$ for all $x, y \in \Gamma$;

(d) a map $\Delta: \Gamma \rightarrow \Gamma_{R} \otimes \Gamma_{R}$ which is a 2-multimorphism of $R$-bimodules, which is is coassociative and cocommutative with counit $\epsilon$, and such that

$$
\Delta(x y)=\sum x_{i}^{\prime} y_{j}^{\prime} \otimes x_{i}^{\prime \prime} y_{j}^{\prime \prime},
$$

where $\Delta(x)=\sum x_{i}^{\prime} \otimes x_{i}^{\prime \prime}$ and $\Delta(y)=\sum y_{j}^{\prime} \otimes y_{j}^{\prime \prime}$.

Note that the multiplication on $\Gamma$ descends to a map $\mu: \Gamma_{R} \otimes{ }_{R} \Gamma \rightarrow \Gamma$.

The functor $H_{\Gamma}$ is a comonad, with comonadic structure $\tilde{\eta}: H_{\Gamma} \rightarrow I$ and $\tilde{\mu}: H_{\Gamma} \rightarrow$ $H_{\Gamma} H_{\Gamma}$ induced by $\eta$ and $\mu$. The maps $\epsilon$ and $\Delta$ induce natural transformations

$$
\tilde{\epsilon}: R \rightarrow H_{\Gamma}(R), \quad \tilde{\Delta}: H_{\Gamma}(M) \otimes_{R} H_{\Gamma}(N) \rightarrow H_{\Gamma}\left(M \otimes_{R} N\right)
$$

which give $H_{\Gamma}$ the structure of a symmetric monoidal functor; this structure is compatible the monad structure, so that $\tilde{\eta}$ and $\tilde{\mu}$ are transformations of monoidal functors.

By a module over the $R$-bialgebra $\Gamma$, we mean a pair $\left(M, \psi_{M}\right)$ consisting of an $R$-module $M$, and a map $\psi_{M}: M \rightarrow H_{\Gamma}(M)$ of $R$-modules such that $\tilde{\eta} \psi_{M}=\mathrm{id}_{M}$ and 
$H_{\Gamma}\left(\psi_{M}\right) \psi_{M}=\tilde{\mu} \psi_{M}$. That is, a module is defined to be a coalgebra for the comonad $\left(H_{\Gamma}, \tilde{\eta}, \tilde{\mu}\right)$. Let $\operatorname{Mod}_{\Gamma}$ denote the category of modules over $\Gamma$.

Note that what we are calling a module over $\Gamma$ really does coincide with the usual notion of a right $\Gamma$-module: the map $\psi_{M}: M \rightarrow H_{\Gamma}(M)$ is adjoint to a map $M_{R} \otimes{ }_{R} \Gamma \rightarrow M$ defining a right $\Gamma$-module structure on $M$.

\subsection{Symmetric monoidal structure on $\operatorname{Mod}_{\Gamma}$}

There is a canonical $\Gamma$-module structure on $R$, defined by taking $\psi_{R}=\tilde{\epsilon}: R \rightarrow$ $H_{\Gamma}(R)$.

Given $M, N \in \operatorname{Mod}_{\Gamma}$ we define their tensor product to be $\left(M \otimes_{R} N, \psi_{M \otimes_{R} N}\right)$, where

$$
\psi_{M \otimes_{R} N} \stackrel{\text { def }}{=} \tilde{\Delta}\left(\psi_{M} \otimes \psi_{N}\right): M \otimes_{R} N \rightarrow H_{\Gamma}\left(M \otimes_{R} N\right) .
$$

That is is indeed a $\Gamma$-module follows from the fact that the comonad structure maps $\tilde{\eta}$ and $\tilde{\mu}$ are compatible with monoidal structures.

Proposition 5.2. The above defines a symmetric monoidal structure on the category $\operatorname{Mod}_{\Gamma}$, such that the forgetful functor $U: \operatorname{Mod}_{\Gamma} \rightarrow \operatorname{Mod}_{R}$ is a symmetric monoidal functor.

\section{4. $\Gamma$-algebras}

Let $\Gamma$ be an $R$-bialgebra. By a $\Gamma$-algebra, we mean a commutative monoid object $B$ in the symmetric monoidal category of $\Gamma$-modules. That is, $B$ is equipped with maps $i: R \rightarrow B$ and $m: B \otimes B \rightarrow B$ of $\Gamma$-modules which also provide it with the structure of a commutative ring. We write $\mathrm{Alg}_{\Gamma}$ for the category of $\Gamma$-algebras.

The free $\Gamma$-algebra on an $R$-module $M$ is seen to have the form $\operatorname{Sym}_{R}^{*}\left(M \otimes_{R} \Gamma\right)$, where the symmetric powers are taken with respect to the right $R$-module structure on $\Gamma$.

The forgetful functor $\operatorname{Alg}_{\Gamma} \rightarrow \operatorname{Alg}_{R}$ is plethyistic, in the sense of $\S 4.10$.

\subsection{Graded bialgebras and graded affine category schemes}

A grading for an $R$-bialgebra $\Gamma$ is a decomposition $\Gamma \approx \bigoplus_{k \geqslant 0} \Gamma[k]$, such that

$$
\mu(\Gamma[k] \otimes \Gamma[\ell]) \subseteq \Gamma[k+\ell], \quad \eta(R) \subseteq \Gamma[0], \quad \Delta(\Gamma[k]) \subseteq \Gamma[k]_{R} \otimes \Gamma[k]_{R} .
$$

Thus, in particular, $\Gamma$ is a graded ring, and each graded piece $\Gamma[k]$ is a cocommutative coalgebra.

Suppose that each $\Gamma[k]$ is projective and finitely generated as a right $R$-module. Let $A[k]=H_{\Gamma[k]}(R)$. The $A[k]$ 's admit two different $R$-module structures, which we call the "source" and "target" module structures; the "source" module structure is defined by $(r \cdot s)(x)=f(x r)$, while the "target" module structure is defined by $\left(r \cdot{ }_{t} f\right)(x)=f(r x)$, where $f \in A_{k}, r \in R, x \in \Gamma[k]$. 
We define

$$
\begin{array}{rlrl}
\mu & : A[k] \otimes_{R} A[k] \rightarrow A[k], & \mu(f \otimes g)(x) & =\sum f\left(x_{i}^{\prime}\right) g\left(x_{i}^{\prime \prime}\right), \\
s^{*}: R \rightarrow A[k], & s^{*}(r)(x) & =\epsilon(x r)=\epsilon(x) r, \\
t^{*}: R \rightarrow A[k], & t^{*}(r)(x) & =\epsilon(r x), \\
i^{*}: A[0] \rightarrow R, & i^{*}(f) & =f(\eta(1)), \\
c^{*}: A[k+\ell] \rightarrow A[k]_{s} \otimes_{R} t A[\ell], & \sum f_{i}^{\prime \prime}\left(f_{i}^{\prime}(x) y\right) & =f(x y),
\end{array}
$$

where $\Delta(x)=\sum x_{i}^{\prime} \otimes x_{i}^{\prime \prime}$ and we write $c^{*}(f)=\sum f_{j}^{\prime} \otimes f_{j}^{\prime \prime}$. The map $\mu$ makes $A[k]$ into a commutative ring, and the "source" and "target" $R$-module structures on $A[k]$ are are the same as those induced by the ring homomorphisms $s^{*}$ and $t^{*}$ :

$$
r \cdot s=f(x r)=\mu\left(s^{*}(r) \otimes f\right), \quad r \cdot{ }_{t} f=f(r x)=\mu\left(t^{*}(r) \otimes f\right) .
$$

By a graded category, we mean a small category $C$ equipped with a degree function $\operatorname{deg}$ : $\operatorname{mor} C \rightarrow \mathbb{N}$, such that (i) $\operatorname{deg}(\alpha \circ \beta)=\operatorname{deg}(\alpha)+\operatorname{deg}(\beta)$, and $\operatorname{deg}($ id $)=0$. The data $\mathcal{A}=\left(R, A[k], s^{*}, t^{*}, i^{*}, c^{*}\right)$ described above determine a graded affine category scheme; that is, they corepresent a functor from commutative rings to graded categories. In particular, $R$ represents the set of objects of $C$, and $A[r]$ represents the set of morphisms of $C$ of degree $r$. The maps $s^{*}, t^{*}, i^{*}, c^{*}$ correspond to source, target, identity, and composition in $C$.

If $\mathcal{A}=\left(R, A[r], s^{*}, t^{*}, i^{*}, c^{*}\right)$ is the data representing an affine graded category scheme over $R$, then let $V: \operatorname{Mod}_{R} \rightarrow \operatorname{Mod}_{R}$ be the functor defined by

$$
V(M) \stackrel{\text { def }}{=} \prod_{r \geqslant 0} A[r]_{s} \otimes_{R} M,
$$

where $V(M)$ obtains its $R$-module structure from the "target" module structure on the $A[r]$ 's. The functor $V$ admits the structure of a comonad, in an apparent way; the counit map $V M \rightarrow M$ is the projection $V M \rightarrow A[0]_{s} \otimes_{R} M \stackrel{i^{*} \otimes \mathrm{id}}{\longrightarrow} R \otimes_{R} M \approx M$, and the comultiplication $V M \rightarrow V V M$ is the map

$$
\prod_{r} A[r]_{s} \otimes_{R} M \rightarrow \prod_{\ell} A[k]_{s} \otimes_{R} t\left(\prod_{k} A[\ell]_{s} \otimes M\right) \approx \prod_{k, \ell} A[k]_{s} \otimes_{R} A[\ell]_{s} \otimes_{R} M
$$

induced by the maps $c^{*}$. A coalgebra for the comonad $V$ is called an $\mathcal{A}$-comodule, and the category of $\mathcal{A}$-comodules is denoted $\operatorname{Comod}_{\mathcal{A}}$.

Under our hypothesis on $\Gamma$, the natural transformation $\chi: V(M) \rightarrow H_{\Gamma}(M)$ of functors $\operatorname{Mod}_{R} \rightarrow \operatorname{Mod}_{R}$ defined by

$$
\chi(f \otimes m) \mapsto(x \mapsto f(x) m)
$$

is an isomorphism for all $M$, and the comonad structure on $V$ corresponds exactly to the comonad structure on $H_{\Gamma}$. Thus, we get the following.

Proposition 5.3. If $\Gamma$ is a graded bialgebra over $R$ such that each graded piece $\Gamma[k]$ is projective and finitely generated as a right $R$-module, and if $\mathcal{A}$ is the corresponding graded affine category scheme, then there is an isomorphism of categories $\operatorname{Mod}_{\Gamma} \approx$ $\operatorname{Comod}_{\mathcal{A}}$. 


\section{The bialgebra of power operations}

In this section, we are going to construct a twisted commutative $E_{0}$-bialgebra $\Gamma$, together with a symmetric object $\omega$ in $\operatorname{Mod}_{\Gamma}$; the bialgebra $\Gamma$ will come with a grading in the sense of (5.5).

From the point of view of topology, $\Gamma$ is the "algebra of power operations in Morava E-theory"; more precisely, it is (up to issues of completion), the algebra of additive operations on $\pi_{0}$ of a $K(n)$-local $E$-algebra spectrum. The difficult part of the construction was given in [Str98]. From the point of view of the theory of plethories, $\Gamma$ is the "additive bialgebra" of the plethory associated to $\mathbb{T}$, as described in [BW05, §10].

\subsection{Additive operations on $\mathbb{T}$-algebras}

Let $P=\operatorname{Hom}_{\mathrm{Alg}_{\mathbb{T}}^{*}}\left(\mathbb{T}\left(E_{*}\right), \mathbb{T}\left(E_{*}\right)\right)$. The set $P$ has the structure of a monoid, under composition of morphisms. Because there is a natural bijection $\operatorname{Hom}_{\operatorname{Alg}_{\mathbb{T}}^{*}}\left(\mathbb{T}\left(E_{*}\right), A\right) \approx$ $A_{0}$, defined by evaluation at the canonical generator $\iota \in \mathbb{T}\left(E_{0}\right)$, we see that $P$ is naturally identified with the monoid of endomorphisms of the forgetful functor $\mathrm{Alg}_{\mathbb{T}} \rightarrow \mathrm{Set}$ which sends to $A$ to the underlying set $A_{0}$ of the degree 0 part of $A$. In particular, evaluation at $\iota \in \mathbb{T}\left(E_{0}\right)$ defines a natural bijection

$$
\pi: P \rightarrow\left[\mathbb{T}\left(E_{*}\right)\right]_{0}
$$

to the degree 0 part of $\mathbb{T}\left(E_{*}\right)$. We will use this bijection implicitly in what follows.

Write $\circ: P \times P \rightarrow P$ for the monoid product on $P$, defined in terms of composition of endomorphisms of the forgetful functor. If $f, g \in P$ correspond to maps $\tilde{f}, \tilde{g}: E_{0} \rightarrow$ $\mathbb{T} E_{0}$ of $E_{0}$-modules, then $f \circ g$ corresponds to

$$
E_{0} \stackrel{\tilde{g}}{\rightarrow} \mathbb{T} E_{0} \stackrel{\mathbb{T} \tilde{f}}{\longrightarrow} \mathbb{T} \mathbb{T} E_{0} \stackrel{\mu}{\longrightarrow} \mathbb{T} E_{0}
$$

Observe that if $f \in \mathbb{T}_{m} E_{0}$ and $g \in \mathbb{T}_{n} E_{0}$, then $f \circ g \in \mathbb{T}_{m n} E_{0}$; this is a consequence of the fact that $\mu: \mathbb{T} \mathbb{T} \rightarrow \mathbb{T}$ carries $\mathbb{T}_{m} \mathbb{T}_{n}$ into $\mathbb{T}_{m n}(\S 4.11)$.

The set $P$ has the structure of an abelian group, by addition of natural endomorphisms of the forgetful functor, or equivalently by addition in $\mathbb{T}\left(E_{*}\right)$. We observe that $\circ$ is "left additive", in the sense that

$$
(f+g) \circ h=(f \circ h)+(g \circ h)
$$

for $f, g, h \in P$.

\subsection{The additive bialgebra associated to $\mathbb{T}$}

Let $\Gamma \subset P$ denote the subset of elements $f \in P$ which induce additive natural endomorphisms of the forgetful functor; that is, $f \in \Gamma$ if it induces an endomorphism of the forgetful functor $\mathrm{Alg}_{\mathbb{T}}^{*} \rightarrow \mathrm{Ab}$. Thus, $\Gamma$ is naturally an associative ring, isomorphic to the ring of endomorphisms of the forgetful functor $\mathrm{Alg}_{\mathbb{T}}^{*} \rightarrow \mathrm{Ab}$ to abelian groups.

Define

$$
\Delta^{+}: \mathbb{T}\left(E_{*}\right) \rightarrow \mathbb{T}\left(E_{*}\right) \otimes \mathbb{T}\left(E_{*}\right)
$$

to be the composite

$$
\mathbb{T}\left(E_{0}\right) \stackrel{\mathbb{T}(\nabla)}{\longrightarrow} \mathbb{T}\left(E_{0} \oplus E_{0}\right) \stackrel{\gamma^{-1}}{\sim} \mathbb{T}\left(E_{0}\right) \otimes \mathbb{T}\left(E_{0}\right),
$$


where $\Delta: E_{*} \rightarrow E_{*} \oplus E_{*}$ denotes the diagonal. Say an element $f \in\left[\mathbb{T}\left(E_{*}\right)\right]_{0}$ is primitive with respect to $\circ$ if $\Delta^{+}(f)=f \otimes 1+1 \otimes f$. It is a straightforward exercise to check that under the bijection $P \stackrel{\sim}{\longrightarrow}\left[\mathbb{T}\left(E_{*}\right)\right]_{0}$, elements of $\Gamma$ correspond precisely to primitive elements.

Translating this into topology by means of the isomorphism $\mathbb{T}_{m}\left(E_{*}\right) \approx E_{*}^{\wedge} B \Sigma_{m}$, we see that $\Gamma \approx \bigoplus_{k \geqslant 0} \Gamma[k]$, where $\Gamma[k] \subset \mathbb{T}_{p^{k}}\left(E_{0}\right)$ defined by

$$
0 \rightarrow \Gamma[k] \rightarrow E_{0}^{\wedge} B \Sigma_{p^{k}} \rightarrow \bigoplus_{0<j<p^{k}} E_{0}^{\wedge} B\left(\Sigma_{j} \times \Sigma_{p^{k}-j}\right)
$$

where the maps are induced by transfer map associated to the evident inclusions $\Sigma_{j} \times \Sigma_{p^{k}-j} \rightarrow \Sigma_{p^{k}}$. (For a symmetric group of order not a $p$ th power, the kernel of these transfer maps is 0 .)

Proposition 6.1. The submodule $\Gamma[k] \subset \mathbb{T}_{p^{k}}\left(E_{0}\right)$ is a direct summand. In particular, $\Gamma[k]$ is a finitely generated free $E_{0}$-module.

Proof. By standard considerations involving Sylow subgroups of $\Sigma_{p^{k}}$, it is straightforward to show that for $k \geqslant 1, \Gamma[k]$ is the kernel of the single transfer map $E_{0}^{\wedge} B \Sigma_{p^{k}} \rightarrow$ $E_{0}^{\wedge} B\left(\Sigma_{p^{k-1}}\right)^{p}$. Strickland [Str98] proves that cokernel of the cohomology transfer is a finite free $E_{0}$-module, so that there is an exact sequence

$$
E^{0} B\left(\Sigma_{p^{k-1}}\right)^{p} \stackrel{\tau}{\rightarrow} E^{0} B \Sigma_{p^{k}} \stackrel{\pi}{\rightarrow} R_{p^{k}} \rightarrow 0
$$

where $R_{p^{k}}$ is a finite free $E_{0}$-module. Taking $E_{0}$-linear duals gives the desired result.

Observe that $\Gamma[k] \circ \Gamma[\ell] \subseteq \Gamma[k+\ell]$, whence $\Gamma$ is a graded ring. The unit $1 \in \Gamma$ corresponds to the canonical generator $\iota \in \mathbb{T}_{1}\left(E_{*}\right)$. From now on we omit the "०" notation when discussing the product on $\Gamma$.

The map $E_{0} \rightarrow P$ defined by $r \mapsto r \cdot 1$ factors through a map $\eta: E_{0} \rightarrow \Gamma$, so that $\eta(r)=\pi\left(\mathbb{T}\left(\lambda_{r}\right)\right)$, where $\lambda_{r}: E_{0} \rightarrow E_{0}$ denotes multiplication by $r \in E_{0}$. The map $\eta: E_{0} \rightarrow \Gamma$ is a ring homomorphism. Observe that the image of $\eta$ need not be central in $\Gamma$ (and in fact it is not, except when $k=\mathbb{F}_{p}$ and $n=1$.)

The ring $\Gamma$ becomes a $E_{0}$-bimodule by $\eta$, and multiplication descends to a map $\mu: \Gamma_{E_{0}} \otimes E_{0} \Gamma \rightarrow \Gamma$.

Let $\epsilon^{\times}: P \rightarrow E_{0}$ and $\Delta^{\times}: P \rightarrow P \otimes P$ be the maps isomorphic to the morphisms of $\mathbb{T}$-algebras

$$
\mathbb{T}\left(E_{0}\right) \rightarrow E_{0}, \quad \mathbb{T}\left(E_{0}\right) \rightarrow \mathbb{T}\left(E_{0} \oplus E_{0}\right),
$$

which correspond to the maps

$$
E_{0} \stackrel{\text { id }}{\longrightarrow} E_{0}, \quad E_{0} \stackrel{\sim}{\longrightarrow} \mathbb{T}_{1}\left(E_{0}\right) \otimes \mathbb{T}_{1}\left(E_{0}\right) \subset \mathbb{T}\left(E_{0}\right) \otimes \mathbb{T}\left(E_{0}\right)
$$

of $E_{0}$-modules. (In terms of topology, $\Delta^{\times}$and $\epsilon^{\times}$are induced by the space-level diagonal and projection maps $B \Sigma_{m} \rightarrow B \Sigma_{m} \times B \Sigma_{m}$ and $B \Sigma_{m} \rightarrow *$.)

Proposition 6.2. The maps $\epsilon^{\times}$and $\Delta^{\times}$restrict to functions

$$
\epsilon: \Gamma \rightarrow E_{0}, \quad \Delta: \Gamma \rightarrow \Gamma_{E_{0}} \otimes \Gamma_{E_{0}}
$$

and the data $(\Gamma, \epsilon, \Delta, \eta, \mu)$ constitutes a graded twisted commutative $E_{0}$-bialgebra. 
Proof. The map $\Gamma_{E_{0}}^{\otimes 2} \rightarrow P^{\otimes 2}$ is an inclusion, by (6.1). Its image is the set of "interlinear elements"; an element $f \in P \otimes P$ is interlinear if the induced operation $\bar{f}: A \times$ $A \rightarrow A$ on an $\mathbb{T}$-algebra $A$ is additive in each variable, and if $\bar{f}(x c, y)=\bar{f}(x, y c)$ for all $c \in E_{0}$; see [BW05, Prop. 10.2] for a proof. It is clear that $\Delta^{\times}$carries $\Gamma$ into the set of interlinear elements, and thus we obtain the desired map $\Delta$. The rest of the argument is as in [BW05].

Note that the diagonal map sends each graded piece to a single grading; that is, $\Delta(\Gamma[k]) \subseteq \Gamma[k]_{E_{0}} \otimes \Gamma[k]_{E_{0}}$. Thus, $\epsilon$ and $\Delta$ give $\Gamma[k]$ a cocommutative coalgebra structure, dual to the ring structure on $E^{0} B \Sigma_{p^{k}} /($ transfer). Thus, $\Gamma \approx \bigoplus \Gamma[k]$ is a graded bialgebra, in the sense of $\S 5.5$.

Example 6.3. Let $G_{0}$ be the multiplicative group over a perfect field $k$ of characteristic $p$, and let $E$ be the corresponding Morava $E$-theory. In this case, $E$ is the extension of $p$-complete $K$-theory to the Witt ring $\mathbb{W}_{k}$. Let $a \mapsto a^{\sigma}$ be the lift to $\mathbb{W}_{k}$ of the Frobenius automorphism of $k$. In this case, $\Gamma$ is isomorphic to the ring $\mathbb{W}_{k}\left\langle\psi^{p}\right\rangle$, where $\psi^{p}$ is the $p$ th Adams operation (viewed as a power operation on $E$-algebras), which satisfies the commutation relation

$$
\psi^{p} \cdot a=a^{\sigma} \cdot \psi^{p} \quad \text { for } a \in \mathbb{W}_{k} .
$$

Observe that $\mathbb{W}_{k}$ is not central in $\Gamma$ (unless $k \approx \mathbb{F}_{p}$.) The grading on $\Gamma$ is such that $\Gamma[0]=\mathbb{W}_{k}$, and $\psi^{p} \in \Gamma[1]$. Since $\psi^{p}$ is a multiplicative operation, we have

$$
\epsilon\left(\psi^{p}\right)=1, \quad \Delta\left(\psi^{p}\right)=\psi^{p} \otimes \psi^{p} .
$$

Let $\operatorname{Alg}_{\Gamma}$ denote the category of $\Gamma$-algebras. We define a functor $U: \operatorname{Alg}_{\mathbb{T}} \rightarrow \operatorname{Alg}_{\Gamma}$ by

$$
U(B)=\operatorname{Hom}_{\mathrm{Alg}}(P, B),
$$

equipped with the $\Gamma$-algebra structure induced by the action of $\Gamma$ on $P$. The underlying $E_{0}$-module of $U B$ is just the underlying $E_{0}$-module of $B$.

\subsection{The $\Gamma$-module $\omega$}

Let $\omega$ be the object in $\operatorname{Mod}_{\Gamma}$ defined by the reduced cohomology of $\mathbb{C P}^{1} \approx S^{2}$ :

$$
\omega=\operatorname{Ker}\left[U\left(E^{0}\left(\mathbb{C P}^{1}\right)\right) \rightarrow U\left(E^{0}\right)\right] .
$$

It is free of rank 1 as an $E_{0}$-module, and thus is a symmetric object in $\operatorname{Mod}_{\Gamma}$. This is a slight abuse of notation; we already use $\omega$ to represent the kernel of $E^{*}\left(\mathbb{C P}^{1}\right) \rightarrow E^{*}$ in the category of $E_{*}$-modules.

\section{Operation algebras for non-zero gradings}

In the previous section we constructed a bialgebra $\Gamma$, which acts naturally on the even degree part of a $\mathbb{T}$-algebra. In this section we describe what happens when we take odd gradings into account. In the end, we will show that underlying an $\mathbb{T}$-algebra is an object of $\operatorname{Mod}_{\Gamma}^{*}$, the $\mathbb{Z} / 2$-graded $\omega$-twisted tensor category of modules over $\Gamma$, in 
terms of the formalism of $\S 2$. We will define $\operatorname{Alg}_{\Gamma}^{*}$ to be the category of commutative ring objects in $\operatorname{Mod}_{\Gamma}^{*}$, and will obtain a "forgetful" functor

$$
U: \operatorname{Alg}_{\mathbb{T}}^{*} \rightarrow \operatorname{Alg}_{\Gamma}^{*} .
$$

The functor $U$ is plethyistic (7.18), in the sense of $\S 4.10$.

To obtain this result, we will first set up a $\mathbb{Z}$-graded theory. In particular, for each $q \in \mathbb{Z}$, we will construct $\Gamma^{q}$ as a set of additive operations sitting inside $P^{q} \approx$ $\operatorname{Hom}_{\mathrm{Alg}_{\mathrm{T}}^{*}}\left(\mathbb{T}\left(\omega^{q / 2}\right), \mathbb{T}\left(\omega^{q / 2}\right)\right) \approx \bigoplus \pi_{-q} L \mathbb{P}_{m}\left(\Sigma^{-q} E\right)$, analogously to the construction of $\Gamma$ in $\S 6$. The ring $\Gamma^{q}$ naturally acts on $\pi_{-q}$ of a $K(n)$-local $E$-algebra. The collection of $\left\{\Gamma^{q}\right\}$ forms a kind of "ZZ-graded bialgebra". In particular, there will be coproduct maps

$$
\Delta_{i, j}: \Gamma_{E_{0}}^{i+j} \rightarrow \Gamma_{E_{0}}^{i} \otimes \Gamma_{E_{0}}^{j}
$$

which determine tensor functors

$$
\otimes: \operatorname{Mod}_{\Gamma^{i}} \times \operatorname{Mod}_{\Gamma^{j}} \rightarrow \operatorname{Mod}_{\Gamma^{i+j}} .
$$

Furthermore, there are "forgetful" functors $\tilde{U}^{i}: \operatorname{Alg}_{\mathbb{T}}^{*} \rightarrow \operatorname{Mod}_{\Gamma^{i}}$. These will fit together into a tensor functor

$$
\tilde{U}^{*}: \operatorname{Alg}_{\mathbb{T}}^{*} \rightarrow \operatorname{Mod}_{\tilde{U}^{*} E_{*}}^{\mathbb{Z}},
$$

where the target is a category of " $\mathbb{Z}$-graded $\tilde{U}^{*} E_{*}$-modules" built from the categories $\operatorname{Mod}_{\Gamma^{i}}(\operatorname{see} \S 7.2)$.

Two features allow us to reinterpret this structure in terms of the $\mathbb{Z} / 2$-graded formalism of $\S 2$. First, the periodicity of the theory $E_{*}$ produces natural equivalences $\operatorname{Mod}_{\Gamma^{i}} \approx \operatorname{Mod}_{\Gamma^{i+2}}$ (7.8). Second, the "suspension map" defines an isomorphism $\Gamma^{1} \rightarrow$ $\Gamma^{0}$ of rings (7.13). Using these observations, we reformulate the category $\operatorname{Mod}_{\tilde{U}^{*} E_{*}}^{\mathbb{Z *}}$ in terms of the bialgebra $\Gamma$, by producing (in $\S 7.6$ ) an equivalence of tensor categories between $\operatorname{Mod}_{\tilde{U}^{*} E_{*}}^{\mathbb{Z} *}$ and the $\mathbb{Z} / 2$-graded $\omega$-twisted category $\operatorname{Mod}_{\Gamma}^{*}$ of $\Gamma$-modules.

\subsection{The rings $\Gamma^{q}$}

Let $P^{q}=\operatorname{Hom}_{\operatorname{Mod}_{E_{*}}}\left(\omega^{q / 2}, \mathbb{T}\left(\omega^{q / 2}\right)\right) \approx \operatorname{Hom}_{\operatorname{Alg}_{\mathbb{T}}^{*}}\left(\mathbb{T}\left(\omega^{q / 2}\right), \mathbb{T}\left(\omega^{q / 2}\right)\right)$. As in $\S 6.1$, there is a natural identification of $P^{q}$ with the monoid of endomorphisms of the functor

$$
A \mapsto \operatorname{Hom}_{\operatorname{Mod}_{E_{*}}}\left(\omega^{q / 2}, A\right): \operatorname{Alg}_{\mathbb{T}}^{*} \rightarrow \text { Set. }
$$

We let $\Gamma^{q} \subset P^{q}$ denote the subset of elements which correspond to additive endomorphisms. Thus $\Gamma^{q}$ is isomorphic to the ring of endomorphisms of the functor $A \mapsto \operatorname{Hom}_{\operatorname{Mod}_{E_{*}}}\left(\omega^{q / 2}, A\right): \operatorname{Alg}_{\mathbb{T}}^{*} \rightarrow \mathrm{Ab}$.

Define $\Delta^{+}: P^{q} \rightarrow P^{q} \otimes P^{q}$ by postcomposition with the map

$$
\gamma^{-1} \circ \mathbb{T}(\nabla): \mathbb{T}\left(\omega^{q / 2}\right) \rightarrow \mathbb{T}\left(\omega^{q / 2}\right) \otimes \mathbb{T}\left(\omega^{q / 2}\right) .
$$

As in $\S 6.2$, we see that $\Gamma^{q}$ corresponds to the set of primitives in $P^{q}$ with respect to $\Delta^{+}$. Observe that $\Gamma^{0}$ is just the ring $\Gamma$ of $\S 6$.

Furthermore, we have the following.

Proposition 7.1. There are non-canonical isomorphisms of rings $\Gamma^{q} \approx \Gamma^{q+2 k}$ for all $q, k \in \mathbb{Z}$. 
Proof. This is immediate from the definitions, and the fact that $\omega^{q / 2} \approx \omega^{(q+2 k) / 2}$ as $E_{*}$-modules.

In terms of topology, $\Gamma^{q}=\bigoplus_{k \geqslant 0} \Gamma^{q}[k]$, where $\Gamma^{q}[k] \subseteq \operatorname{Hom}_{\operatorname{Mod}_{E_{*}}}\left(\omega^{q / 2}, \mathbb{T}_{p^{k}}\left(\omega^{q / 2}\right)\right)$ can be identified with $\operatorname{Hom}_{\operatorname{Mod}_{E_{*}}}\left(E_{0}^{\wedge} S^{-q}, K^{q}[k]\right)$, where $K^{q}[k]$ fits in the exact sequence

$$
0 \rightarrow K^{q}[k] \rightarrow E_{*}^{\wedge} B \Sigma_{p^{k}}^{-q \rho_{p^{k}}} \rightarrow \bigoplus_{0<j<p^{k}} E_{*}^{\wedge} B\left(\Sigma_{j} \times \Sigma_{p^{k}-j}\right)^{-q \rho_{p^{k}}}
$$

defined using transfer, where $\rho_{m}$ denotes the real ( $m$-dimensional) permutation representation of $\Sigma_{m}$. In particular, it is natural to regard $\Gamma^{q}[k]$ as a submodule of $E_{-q}^{\wedge}\left(B \Sigma_{p^{k}}^{-q \rho_{p^{k}}}\right) \approx E_{0}^{\wedge}\left(B \Sigma_{p^{k}}^{-q \bar{\rho}_{p^{k}}}\right)$, where $\bar{\rho}_{p^{k}} \subset \rho_{p^{k}}$ denotes the reduced real permutation representation.

Proposition 7.2. For all $q \in \mathbb{Z}$, the inclusion of $\Gamma^{q}[k]$ in $\operatorname{Hom}_{\operatorname{Mod}_{E_{*}}}\left(\omega^{q / 2}, \mathbb{T}_{p^{k}}\left(\omega^{q / 2}\right)\right)$ is the inclusion of a direct summand, whence $\Gamma^{q}[k]$ is a finite free $E_{0}$-module.

Proof. (Compare [Str98, Prop. 5.6].) We have already addressed the case of $q=0$ (6.1), and thus need only consider $q=1$. Let $B_{*} \stackrel{\text { def }}{=} \bigoplus_{m \geqslant 0} \pi_{*} L \mathbb{P}_{m} \Sigma E$; this is a commutative Hopf algebra, with product given by the ring structure of $\mathbb{P} \Sigma E$, and coproduct given by $\mathbb{P}(\nabla): \mathbb{P} \Sigma E \rightarrow \mathbb{P}(\Sigma E \vee \Sigma E)$. The result follows from the observation that $B_{*}$ is a primitively generated exterior algebra, since $B_{*} \approx \mathbb{T}\left(\omega^{1 / 2}\right)$. We prove this using the bar spectral sequence

$$
E_{2}=\operatorname{Tor}_{*}^{E_{*}}\left(A_{*}, A_{*}\right) \Longrightarrow B_{*},
$$

where $A_{*} \approx \bigoplus_{m \geqslant 0} \pi_{*} L \mathbb{P}_{m} E$. By a result of Strickland, following Kashiwabara [Str98, Prop. 5.1], $A_{*}$ is a polynomial algebra, finite type with respect to the grading determined by $m$. Thus $\operatorname{Tor}_{*}^{E_{*}}\left(A_{*}, A_{*}\right)$ is an exterior algebra, with generators represented by the homology suspensions of the generators of the polynomial ring $A_{*}$. These classes survive, since they detect the image of the suspension maps $\pi_{*} L \mathbb{P}_{m} E \rightarrow$ $\pi_{*+1} L \mathbb{P}_{m} \Sigma E$, and thus the spectral sequence collapses at $E_{2}$.

We will need the following result in $\S 8$.

Proposition 7.3. The inclusion of $\Gamma^{q} \subseteq \operatorname{Hom}_{\operatorname{Mod}_{E_{*}}}\left(\omega^{q / 2}, \mathbb{T}\left(\omega^{q / 2}\right)\right)$ induces an isomorphism

$$
\operatorname{Sym}_{E_{*}}\left(\Gamma^{q} \otimes \omega^{q / 2}\right) \otimes \mathbb{Q} \approx \mathbb{T}\left(\omega^{q / 2}\right) \otimes \mathbb{Q}
$$

of graded $E_{*}$-modules.

Proof. By (7.2), $\Gamma^{q} \otimes \omega^{q / 2}$ is the primitives of the Hopf algebra $\mathbb{T}\left(\omega^{q / 2}\right)$, and is a direct summand. Thus, $\left(\Gamma^{q} \otimes \omega^{q / 2}\right) \otimes \mathbb{Q}$ is the primitives of $\mathbb{T}\left(\omega^{q / 2}\right) \otimes \mathbb{Q}$. The result follows from the structure theory of graded Hopf algebras.

Remark 7.4. It may be helpful to consider the following picture. Fix $k \geqslant 1$, and consider $\operatorname{Prim}_{q} \longmapsto E_{q}^{\wedge} B \Sigma_{p^{k}}^{q \rho_{p^{k}}} \approx \pi_{q} L \mathbb{P}_{p^{k}} \Sigma^{q} E \rightarrow \operatorname{Ind}_{q}$, where $\operatorname{Prim}_{q}$ and $\operatorname{Ind}_{q}$ denote the part of the primitives and indecomposables of $\bigoplus_{m} \pi_{*} L \mathbb{P}_{m} \Sigma^{q} E$ associated the $m=p^{k}$ summand in dimension $q$; the $\operatorname{ring} \Gamma^{q}$ is the direct sum of the $E_{0}$-modules Prim $_{-q}$ as $k$ varies. The suspension map $\pi_{q} L \mathbb{P}_{p^{k}} \Sigma^{q} E \rightarrow \pi_{q+1} L \mathbb{P}_{p^{k}} \Sigma^{q+1} E$ factors 
through $\operatorname{Prim}_{q} \rightarrow \operatorname{Ind}_{q+1}$; from these maps we obtain the following diagram, which is 2-periodic.

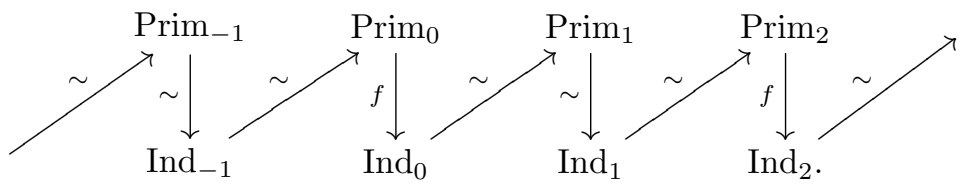

The vertical maps $\operatorname{Prim}_{q} \rightarrow \operatorname{Ind}_{q}$ are isomorphisms for odd $q$ using (7.2). The map $\operatorname{Ind}_{0} \rightarrow \operatorname{Prim}_{2}$ is an isomorphism by Theorems 8.5 and 8.6 of [Str98], where the result is stated in "dual" form; specifically, in that paper it is proved that the image of $\operatorname{Prim} E^{0} B \Sigma_{p^{k}} \rightarrow \operatorname{Ind} E^{0} B \Sigma_{p^{k}}$ is generated by the Euler class of $\bar{\rho}_{p^{k}}^{\mathbb{C}}$. The map $f$ is not an isomorphism, but is a monomorphism with torsion cokernel; the present paper is a essentially a meditation on $\operatorname{cok} f$.

The bijection $\operatorname{Hom}_{\operatorname{Alg}_{*}^{*}}\left(\mathbb{T}\left(\omega^{q / 2}\right), \mathbb{T}\left(\omega^{q / 2}\right)\right) \rightarrow P^{q}$ induces an associative monoid structure on $P^{q}$, defined by composition, and thus descends to a multiplication on $\Gamma^{q}$. There is a ring homomorphism $\eta_{q}: E_{0} \rightarrow \Gamma^{q}$, defined as for $\Gamma$.

Finally, let $\Delta_{i, j}^{\times}: P^{i+j} \rightarrow P^{i} \otimes P^{j}$ be the map induced by the "multiplicativity map" $\nu: \mathbb{T}\left(\omega^{i / 2} \otimes \omega^{j / 2}\right) \rightarrow \mathbb{T}\left(\omega^{i / 2} \oplus \omega^{j / 2}\right)$. As before, we have $\epsilon^{\times}: P^{0} \rightarrow E_{0}$.

Proposition 7.5. The maps $\Delta_{i, j}^{\times}$restrict to maps

$$
\Delta_{i, j}: \Gamma_{E_{0}}^{i+j} \rightarrow \Gamma_{E_{0}}^{i} \otimes \Gamma_{E_{0}}^{j}
$$

which in turn induce functors

$$
\otimes: \operatorname{Mod}_{\Gamma^{i}} \times \operatorname{Mod}_{\Gamma^{j}} \rightarrow \operatorname{Mod}_{\Gamma^{i+j}},
$$

which are unital, associative, and commutative in the sense that there are natural isomorphisms

$$
\begin{gathered}
E_{0} \otimes M \approx M \approx M \otimes E_{0} \quad \text { of functors } \operatorname{Mod}_{\Gamma^{i}} \rightarrow \operatorname{Mod}_{\Gamma^{i}}, \\
M_{1} \otimes\left(M_{2} \otimes M_{3}\right) \approx\left(M_{1} \otimes M_{2}\right) \otimes M_{3},
\end{gathered}
$$

and

$$
M_{1} \otimes M_{2} \approx M_{2} \otimes M_{1},
$$

where $E_{0}$ is regarded as a $\Gamma^{0}$-module. (Note that the interchange map introduces a sign when applied to elements of odd degree.)

Define functors $\tilde{U}^{q}: \operatorname{Alg}_{\mathbb{T}}^{*} \rightarrow \operatorname{Mod}_{\Gamma^{q}}$ for $q \in \mathbb{Z}$ by sending $B \in \operatorname{Alg}_{\mathbb{T}}^{*}$ to the right $\Gamma^{q}$-module $\operatorname{Hom}_{\mathrm{Alg}_{\mathbb{T}}^{*}}\left(\mathbb{T}\left(\omega^{q / 2}\right), B\right) \approx \operatorname{Hom}_{\operatorname{Mod}_{E_{*}}}\left(\omega^{q / 2}, B\right)$.

Remark 7.6. If $A$ is a $K(n)$-local commutative $E$-algebra, so that $\pi_{*} A$ is a $\mathbb{T}$-algebra, then tracing through the definitions reveals that the underlying $E_{0}$-module of $\tilde{U}^{q}\left(\pi_{*} A\right)$ is canonically identified with $\pi_{-q} A$. Thus, we see that $\pi_{-q} A$ naturally carries the structure of a $\Gamma^{q}$-module.

Proposition 7.7. There are natural transformations

$$
\tilde{U}^{i}(M) \otimes \tilde{U}^{j}(N) \rightarrow \tilde{U}^{i+j}(M \otimes N)
$$

of functors $\mathrm{Alg}_{\mathbb{T}}^{*} \times \operatorname{Alg}_{\mathbb{T}}^{*} \rightarrow \operatorname{Mod}_{\Gamma^{i+j}}$. 
Proof. The proof is straightforward.

\section{2. $\quad \mathbb{Z}$-graded $\Gamma^{*}$-modules and $\tilde{U}^{*} E_{*}$-modules}

By a $\mathbb{Z}$-graded $\Gamma^{*}$-module, we mean a tuple $M=\left(M^{i}\right)_{i \in \mathbb{Z}}$, where $M^{i}$ is a right $\Gamma^{i}$-module. We write $\operatorname{Mod}_{\Gamma^{*}}^{\mathbb{Z} *}$ for the category of $\mathbb{Z}$-graded $\Gamma^{*}$-modules. This category admits the structure of an additive symmetric monoidal category, by the tensor product functors of $(7.5)$, so that $M \otimes N=\left(P^{i}\right)_{i \in \mathbb{Z}}$, where $P^{i}=\bigoplus_{q} M^{q} \otimes N^{i-q}$.

Let $\tilde{U}^{*}: \operatorname{Alg}_{\mathbb{T}}^{*} \rightarrow \operatorname{Mod}_{\Gamma^{*}}^{\mathbb{Z}}$ be the functor which sends a $\mathbb{T}$-algebra $B$ to the $\mathbb{Z}$-graded $\Gamma^{*}$-module $\left(\tilde{U}^{i} B\right)$. By (7.7), the functor $\tilde{U}^{*}$ is a lax symmetric monoidal functor; i.e., there is a coherent natural transformation $\tilde{U}^{*} B \otimes \tilde{U}^{*} C \rightarrow \tilde{U}^{*}(B \otimes C)$.

By these remarks, it follows that $\tilde{U}^{*} E_{*}$ is a commutative monoid object in $\operatorname{Mod}_{\Gamma^{*}}^{\mathbb{Z} *}$, and that $\tilde{U}^{*} B$ is tautologically a $\tilde{U}^{*} E_{*}$-module object for all $B$ in $\operatorname{Alg}_{\mathbb{T}}^{*}$. Let $\operatorname{Mod}_{\tilde{U}^{*} E_{*}}^{\mathbb{Z}}$ denote the category of $\tilde{U}^{*} E_{*}$-modules; it is a symmetric monoidal category, with tensor product defined by

$$
M \otimes_{\tilde{U}^{*} E_{*}} N \stackrel{\text { def }}{=} \operatorname{cok}\left[M \otimes \tilde{U}^{*} E_{*} \otimes N \rightrightarrows M \otimes N\right] .
$$

There is an evident forgetful functor $\operatorname{Mod}_{\tilde{U}^{*} E_{*}}^{\mathbb{Z} *} \rightarrow \operatorname{Mod}_{E_{*}}^{\mathbb{Z} *}$ to the $\mathbb{Z}$-graded category of $E_{*}$-modules, and this forgetful functor is strongly symmetric monoidal: the underlying $\mathbb{Z}$-graded $E_{*}$-module of $M \otimes_{\tilde{U}^{*} E_{*}} N$ is just the usual $\mathbb{Z}$-graded tensor product of the underlying $E_{*}$-modules of $M$ and $N$.

Thus, the functor $\tilde{U}^{*}: \operatorname{Alg}_{\mathbb{T}}^{*} \rightarrow \operatorname{Mod}_{\Gamma^{*}}^{\mathbb{Z} *}$ lifts tautologically to a functor

$$
\tilde{U}^{*}: \operatorname{Alg}_{\mathbb{T}}^{*} \rightarrow \operatorname{Mod}_{\tilde{U}^{*} E_{*}}^{\mathbb{Z} *}
$$

Furthermore, it is straightforward to check (by looking at what happens on the underlying $E_{*}$-modules) that the lifted $\tilde{U}^{*}$ is a strong symmetric monoidal functor; i.e., $\tilde{U}^{*} B \otimes_{\tilde{U}^{*} E_{*}} \tilde{U}^{*} C \rightarrow \tilde{U}^{*}(B \otimes C)$ is an isomorphism.

The rest of this section is devoted to giving a more elementary description of $\operatorname{Mod}_{\tilde{U}^{*} E_{*}}^{\mathbb{Z} *}$

\subsection{Periodicity}

Observe that the underlying $E_{0}$-module of $\tilde{U}^{2 i} E_{*}$ is canonically isomorphic to $\pi_{-2 i} E$.

Proposition 7.8. The functor $\operatorname{Mod}_{\Gamma^{i}} \rightarrow \operatorname{Mod}_{\Gamma^{i+2}}$ defined by $M \mapsto M \otimes \tilde{U}^{2} E_{*}$ is an equivalence of categories, and there are natural isos $\tilde{U}^{i}(M) \otimes \tilde{U}^{2} E_{*} \approx \tilde{U}^{i+2}(M)$ of functors $\operatorname{Alg}_{\mathbb{T}}^{*} \rightarrow \operatorname{Mod}_{\Gamma^{i+2}}$.

Proof. The isomorphism $\tilde{U}^{2}\left(E_{0}\right) \otimes \tilde{U}^{-2}\left(E_{0}\right) \approx \tilde{U}^{0}\left(E_{0}\right) \approx E_{0}$ produces an inverse up to isomorphism for this functor.

As a consequence, we note the following.

Lemma 7.9. Let

$$
V^{\prime}: \operatorname{Mod}_{\Gamma^{0}} \times \operatorname{Mod}_{\Gamma^{1}} \rightarrow \operatorname{Mod}_{\tilde{U}^{*} E_{*}}^{\mathbb{Z} *}
$$

be the functor which on objects sends $\left(M^{0}, M^{1}\right)$ to $N^{i}$, where $N^{2 i}=M^{0} \otimes \tilde{U}^{2 i} E_{*}$ and $N^{2 i+1}=M^{1} \otimes \tilde{U}^{2 i} E_{*}$, and $N$ is given a $\tilde{U}^{*} E_{*}$-module structure in the evident way. Then $V^{\prime}$ is an equivalence of categories. 


\subsection{Suspension}

Define functions $e_{q}: \Gamma^{q+i} \rightarrow \Gamma^{i}$ for all $q \geqslant 0$ and all $i \in \mathbb{Z}$ so that $e_{q}(f)$ is the composite

$$
\omega^{i / 2} \approx \omega^{-q / 2} \otimes \omega^{(q+i) / 2} \stackrel{\text { id } \otimes f}{\longrightarrow} \omega^{-q / 2} \otimes \mathbb{T}\left(\omega^{(q+i) / 2}\right) \stackrel{E_{q}}{\longrightarrow} \mathbb{T}\left(\omega^{-q / 2} \otimes \omega^{(q+i) / 2}\right) \approx \mathbb{T}\left(\omega^{i / 2}\right),
$$

where $E_{q}$ is was defined in $\S 4.12$.

Proposition 7.10. The maps $e_{q}: \Gamma^{i+q} \rightarrow \Gamma^{i}$ are homomorphisms of associative rings under $E_{0}$.

Proof. This is a lengthy but straightforward diagram chase, which depends essentially on (4.14).

Proposition 7.11. The diagram

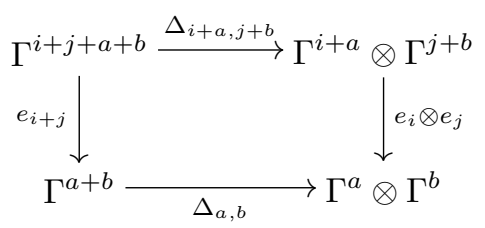

commutes.

Proof. This is a lengthy but straightforward diagram chase, which depends essentially on (4.15).

Let $e_{q}^{*}: \operatorname{Mod}_{\Gamma^{i}} \rightarrow \operatorname{Mod}_{\Gamma^{i+q}}$ denote the functor obtained by restricting along the ring homomorphism $e_{q}$.

Proposition 7.12. There are natural and coherent isomorphisms

$$
e_{a}^{*}(M) \otimes e_{b}^{*}(N) \rightarrow e_{a+b}^{*}(M \otimes N)
$$

of functors $\operatorname{Mod}_{\Gamma^{i}} \times \operatorname{Mod}_{\Gamma^{j}} \rightarrow \operatorname{Mod}_{\Gamma^{i+j+a+b}}$.

Proposition 7.13. The suspension map $e_{1}: \Gamma^{1+2 i} \rightarrow \Gamma^{2 i}$ is an isomorphism for all $i \in \mathbb{Z}$. Thus, the functor $e_{1}^{*}: \operatorname{Mod}_{\Gamma^{2 i}} \rightarrow \operatorname{Mod}_{\Gamma^{1+2 i}}$ is an equivalence of categories.

Proof. By periodicity, this amounts to the observation that $e_{1}: \Gamma^{-1} \rightarrow \Gamma^{-2}$ is an isomorphism. We read this off of the fact that the suspension map $E^{1} B \Sigma_{p^{k}}^{\rho} \rightarrow E^{2} B \Sigma_{p^{k}}^{2 \rho}$ is an isomorphism on primitives, as proved in Strickland and discussed in (7.4).

\section{5. $\mathbb{Z}$-graded $\Gamma^{*}$-modules from spheres}

For $q \geqslant 0$, define a $\mathbb{Z}$-graded $\tilde{U}^{*} E_{*}$-module $\eta(q)$ by

$$
\eta(q) \stackrel{\text { def }}{=} \operatorname{Ker}\left[\tilde{U}^{*}\left(E^{*} S^{q}\right) \rightarrow \tilde{U}^{*}\left(E^{*}(\mathrm{pt})\right)\right] .
$$

Lemma 7.14. There are natural and coherent isomorphisms

$$
\eta(q) \otimes_{\tilde{U}^{*} E_{*}} \eta\left(q^{\prime}\right) \approx \eta\left(q+q^{\prime}\right) .
$$

Proof. Read this off using the Künneth isomorphism

$$
E^{*}\left(S^{q} \times S^{q^{\prime}}\right) \approx E^{*} S^{q} \otimes E^{*} S^{q^{\prime}} .
$$


Clearly, $\eta(0) \approx \tilde{U}^{*} E_{*}$. Observe that $\eta(2)$ is a symmetric object of $\operatorname{Mod}_{\tilde{U}^{*} E_{*}}^{\mathbb{Z} *}$ (in the sense of $\S 2.1)$, and that $\eta(1)$ is an odd square-root of $\eta(2)$.

Proposition 7.15. There is an isomorphism

$$
\eta(q) \approx e_{q}^{*}\left(\tilde{U}^{*} E_{*}\right)
$$

of $\mathbb{Z}$-graded $\Gamma^{*}$-modules.

Proof. This is a straightforward consequence of (3.18).

Proposition 7.16. There are natural isomorphisms

$$
e_{q}^{*} M \approx \eta(q) \otimes_{\tilde{U}^{*} E_{*}} M
$$

of $\mathbb{Z}$-graded $\tilde{U}^{*} E_{*}$-modules.

Proof. Clear from (7.15) and (7.16).

\section{6. $\mathbb{Z} / 2$-graded $\Gamma$-modules}

Let $\operatorname{Mod}_{\Gamma}^{*}$ denote the category of $\mathbb{Z} / 2$-graded $\Gamma$-modules. We give this category a tensor structure using the procedure of $\S 2$, where we twist by the symmetric object $\omega \in \mathrm{Alg}_{\Gamma}$ described above.

Let

$$
V: \operatorname{Mod}_{\Gamma} \rightarrow \operatorname{Mod}_{\tilde{U}^{*} E_{*}}^{\mathbb{Z} *}
$$

denote the functor which on objects sends $M$ to $N$, where $N^{2 i}=M \otimes \tilde{U}^{2 i} E_{*}$ and $N^{2 i+1}=0$. It is clear that $V$ is a strong symmetric monoidal functor. Furthermore, it is clear from the definitions that $V(\omega) \approx \eta(2)$.

Using the method of (2.2), we obtain a symmetric monoidal functor

$$
V^{*}: \operatorname{Mod}_{\Gamma}^{*} \rightarrow \operatorname{Mod}_{\tilde{U}^{*} E_{*}}^{\mathbb{Z} *}
$$

which extends $V$ and sends $\omega^{1 / 2}$ to $\eta(1)$.

Proposition 7.17. The symmetric monoidal functor $V^{*}: \operatorname{Mod}_{\Gamma}^{*} \rightarrow \operatorname{Mod}_{\tilde{U}^{*} E_{*}}^{\mathbb{Z}}$ is an equivalence of categories.

Proof. Explicitly, the functor $V^{*}$ sends an object $M=\left\{M^{0}, M^{1}\right\}$ of $\operatorname{Mod}_{\Gamma}^{*}$ to the object $V\left(M^{0}\right) \oplus V\left(M^{1}\right) \otimes \eta(1)$. By (7.16) this functor is naturally isomorphic to the $M \mapsto V\left(M^{0}\right) \oplus e_{1}^{*} V\left(M_{1}\right)$. Since $e_{1}^{*}: \operatorname{Mod}_{\Gamma^{0}} \rightarrow \operatorname{Mod}_{\Gamma^{1}}$ is an equivalence of categories by (7.13), the result follows using (7.9).

\subsection{The forgetful functor $U$}

Since $V^{*}$ is an equivalence of tensor categories, there is a functor $\tilde{U}: \operatorname{Alg}_{\mathbb{T}}^{*} \rightarrow \operatorname{Mod}_{\Gamma}^{*}$ such that there is a monoidal isomorphism $V^{*} \tilde{U} \approx \tilde{U}^{*}$ of monoidal functors.

Proposition 7.18. The functor $\tilde{U}: \operatorname{Alg}_{\mathbb{T}}^{*} \rightarrow \operatorname{Mod}_{\Gamma}^{*}$ lifts to a functor $U: \operatorname{Alg}_{\mathbb{T}}^{*} \rightarrow \operatorname{Alg}_{\Gamma}^{*}$ which is plethyistic.

Proof. In the sequence of forgetful functors

$$
\operatorname{Alg}_{\mathbb{T}}^{*} \stackrel{U}{\longrightarrow} \operatorname{Alg}_{\Gamma}^{*} \stackrel{U^{\prime}}{\longrightarrow} \operatorname{Alg}_{E_{*}},
$$

the functor $U^{\prime}$ is clearly plethyistic, and the composite $U^{\prime} U$ is plethyistic by (4.12). The result follows. 


\section{Rational algebras}

We say that an object of $\mathrm{Alg}_{\mathbb{T}}^{*}$ (resp. an $E_{*}$-algebra) is rational if the underlying $E_{*}$-module is a (graded) rational vector space. In this section, we prove that if $B$ is an $\mathbb{T}$-algebra, then $\mathbb{Q} \otimes B$ is also an $\mathbb{T}$-algebra in a canonical way; and also that a rational $\mathbb{T}$-algebra is the same thing as a rational $\Gamma$-algebra. Our argument follows that of Knutson [Knu73]. Recall that $U: \operatorname{Alg}_{\mathbb{T}}^{*} \rightarrow \operatorname{Alg}_{E_{*}}$ admits a right adjoint $G$ $(\S 4.10)$.

Lemma 8.1. If $A \in \operatorname{Alg}_{E_{*}}$ is rational, then so is $U G(A)$.

Proof. Let $i=0$ or 1 , and let $X(A)$ denote the $i$ th graded piece of $U G(A)$, regarded as an abelian group. We have that

$$
X(A) \approx \operatorname{Mod}_{E_{*}}\left(\omega^{i / 2}, U G(A)\right) \approx \operatorname{Alg}_{E_{*}}\left(\mathbb{T}\left(\omega^{i / 2}\right), A\right),
$$

where the abelian group structure on $X(A)$ is determined by the coproduct on $\mathbb{T}\left(\omega^{i / 2}\right)$. We need to show that $X(A)$ is rational, and our proof amounts to observing that $X$ is a nilpotent abelian group scheme. More precisely, let $X_{q}(A)$ denote the quotient of the set $X(A)$ under the following equivalence relation; we say that $f$ and $g$ in $X(A)$ are equivalent if their restrictions to $\bigoplus_{k=0}^{q} \mathbb{T}_{k}\left(\omega^{i / 2}\right)$ are equal. Because the coproduct map $\Delta^{+}: \mathbb{T}\left(\omega^{i / 2}\right) \rightarrow \mathbb{T}\left(\omega^{i / 2}\right) \otimes \mathbb{T}\left(\omega^{i / 2}\right)$ restricts to a defined on this summand, $X_{q}(A)$ is in fact a quotient abelian group of $X(A)$. We have that $X_{0}(A)=0$, and

$$
X(A)=\lim _{q} X_{q}(A)
$$

so that it suffices to show that each $X_{q}(A)$ is rational. Furthermore, we have

$$
\operatorname{ker}\left[X_{q}(A) \rightarrow X_{q-1}(A)\right]=\operatorname{Mod}_{E_{*}}\left(M_{q}, A\right),
$$

where $M_{q}=\operatorname{cok}\left[\bigoplus_{0<i<q} \mathbb{T}_{i}\left(\omega^{i / 2}\right) \otimes \mathbb{T}_{q-i}\left(\omega^{i / 2}\right) \rightarrow \mathbb{T}_{q}\left(\omega^{i / 2}\right)\right]$. Since $A$ is rational, this kernel must be rational, and thus inductively we may conclude that $X_{q}(A)$ is rational for all $q$.

Proposition 8.2. Let $B \in \operatorname{Alg}_{\mathbb{T}}^{*}$. There exists a morphism $B \rightarrow B^{\prime} \in \operatorname{Alg}_{\mathbb{T}}^{*}$ which is initial among morphisms from $B$ to rational objects of $\mathrm{Alg}_{\mathbb{T}}^{*}$. Furthermore, the evident map $\mathbb{Q} \otimes U B \rightarrow U B^{\prime}$ is an isomorphism of rational $E_{*}$-algebras.

Proof. Let $\psi: U B \rightarrow(U G)(U B)$ denote the unit of the adjunction. We claim that there is a unique map $\psi^{\prime}: \mathbb{Q} \otimes U B \rightarrow(U G)(\mathbb{Q} \otimes U B)$ making $\mathbb{Q} \otimes U B$ into a coalgebra for the comonad $U G$ such that $U B \rightarrow \mathbb{Q} \otimes U B$ is a map of coalgebras. Consider the diagram

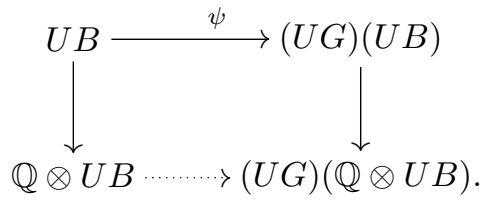

Since $(U G)(\mathbb{Q} \otimes U B)$ is a rational $E_{*}$-algebra by the above, it is clear that there is a unique dotted arrow making the diagram commute. 
Let $\operatorname{Alg}_{\mathbb{T}, \mathbb{Q}}^{*} \subset \operatorname{Alg}_{\mathbb{T}}^{*}$ and $\operatorname{Alg}_{\Gamma, \mathbb{Q}}^{*} \subset \operatorname{Alg}_{\Gamma}^{*}$ denote the full subcategories of rational objects.

Proposition 8.3. Let $B$ be a rational object of $\mathrm{Alg}_{\Gamma}^{*}$. Then there is a unique $\mathbb{T}$-algebra structure on $B$ compatible with the given $\Gamma$-algebra structure.

Proof. It is enough to check this for $S \otimes \mathbb{Q}$, where $S \approx \operatorname{Sym}_{E_{0}}\left(\Gamma \otimes \omega^{q / 2}\right)$ is the free $\Gamma$-algebra on one generator in some degree $q / 2$; observe that $S \otimes \mathbb{Q}$ is clearly an object of $A \lg _{\Gamma}^{*}$. Let $P \approx \mathbb{T}\left(\omega^{q / 2}\right)$ denote the free $\mathbb{T}$-algebra on one generator, and consider the tautological map $f: S \rightarrow P$. By (8.2), we see that $P \otimes \mathbb{Q}$ is a $\mathbb{T}$-algebra. The result follows from the observation that $f \otimes \mathbb{Q}: S \otimes \mathbb{Q} \rightarrow P \otimes \mathbb{Q}$ is an isomorphism (7.3).

Corollary 8.4. The forgetful functor $U: \operatorname{Alg}_{\mathbb{T}}^{*} \rightarrow \operatorname{Alg}_{\Gamma}^{*}$ induces an equivalence of categories $\operatorname{Alg}_{\mathbb{T}, \mathbb{Q}}^{*} \rightarrow \operatorname{Alg}_{\Gamma, \mathbb{Q}}^{*}$.

\section{Critical weights}

Let $U: \mathcal{D} \rightarrow \mathcal{A}$ be a plethyistic functor, where $\mathcal{A}$ is the category of commutative monoid objects in an abelian tensor category $\mathcal{C}$. Let $T: \mathcal{C} \rightarrow \mathcal{C}$ be the monad associated to this situation. Recall from $\S 4.11$ that such a monad comes with maps $\eta: I \rightarrow T$ and $\mu: T T \rightarrow T$ defining the monad structure, and natural maps $\iota: \mathbb{k} \rightarrow T(M)$ and $\delta: T(M) \otimes T(M) \rightarrow T(M)$ defining the commutative ring structure on $T(M)$. We will assume that $T$ is equipped with a weight decomposition, as in $\S 4.11$.

Recall that a collection of morphisms $\left\{f_{j}: A_{j} \rightarrow B\right\}$ in a category called an epimorphic family if for any two morphisms $g, h: B \rightarrow C, g f_{j}=h f_{j}$ for all $f_{j}$ implies $g=h$. We say that $m \geqslant 2$ is a regular weight of $T$ if the family of maps in $\mathcal{C}$

$$
\delta: T_{i}(M) \otimes T_{m-i}(M) \rightarrow T_{m}(M), \quad \mu: T_{d} T_{m / d}(M) \rightarrow T_{m}(M)
$$

is an epimorphic family, where $i$ ranges over integers such that $0<i<m$ and $d$ ranges over divisors of $m$ such that $1<d<m$. In addition, we say that 0 is a regular weight if $\iota: \mathbb{k} \rightarrow T_{0}(M)$ is an epimorphism, and that 1 is a regular weight if $\eta: M \rightarrow T_{1}(M)$ is an epimorphism. We say that $m \geqslant 0$ is a critical weight of $T$ if it is not a regular weight.

The idea of the following proposition is that $T$ is in some sense "generated" by phenomena in critical weights.

Proposition 9.1. Let $\psi: T(M) \rightarrow M$ be a T-algebra structure on $M \in \mathcal{C}$. Let $N \subseteq M$ be a subobject of $M$ in $\mathcal{A}$. If $\psi\left(T_{m}(N)\right) \subseteq N$ for each critical weight $m$, then there is a unique T-algebra structure on $N$ such that the inclusion $N \rightarrow M$ is a morphism of $T$-algebras.

Proof. It suffices to show that the image of the composite $T(N) \rightarrow T(M) \stackrel{\psi}{\longrightarrow} M$ is contained in $N$. We will show by induction on $m \geqslant 0$ that $\psi \circ \alpha_{m}$

$$
T_{m}(N) \stackrel{\alpha_{m}}{\longrightarrow} T_{m}(M) \stackrel{\psi}{\longrightarrow} M
$$

factors through $N$. When $m$ is a critical weight this is true by hypothesis. If $m=0$ is not critical, then $\iota: \mathbb{k} \rightarrow T_{0}(M)$ is epimorphic, and thus $\alpha_{0}$ factors through $N$ since 
$N$ is a subobject. If $m=1$ and is not critical, then $\eta: M \rightarrow T_{1}(M)$ is epimorphic, and thus $\alpha_{1}$ must factor through $N$ since $\psi \circ \eta=\mathrm{id}_{M}$. If $m \geqslant 2$ and is not critical, then there is a unique dotted arrow making the diagrams

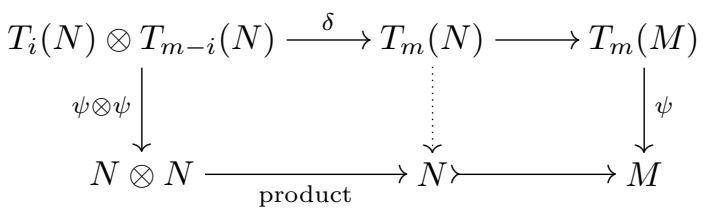

and

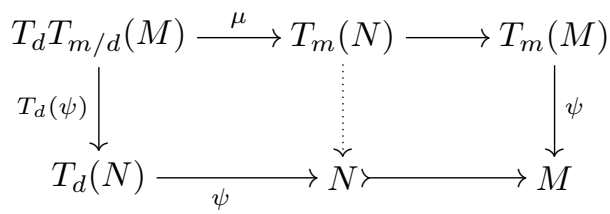

commute.

\section{Proof of the congruence criterion}

In this section we prove Theorem A, using the results of $\S \S 8$ and 9.

\subsection{The only critical weight of $\mathbb{T}$ is $p$}

The proof of the following proposition owes something to McClure's discussion in [BMMS86, Ch. IX].

Proposition 10.1. With respect to the standard weight decompositions, the only critical weight for the algebraic approximation functor $\mathbb{T}$ is $p$.

Proof. We will see later in this section exactly how $p$ is a critical weight. Right now we prove that all other weights are regular.

It is clear that 0 and 1 are regular weights for $\mathbb{T}$, since $\iota: E_{*} \rightarrow \mathbb{T}_{0}\left(M_{*}\right)$ and $\eta: M \rightarrow$ $\mathbb{T}_{1}\left(M_{*}\right)$ are isomorphisms for all $E_{*}$-modules.

To show that $m$ is a regular weight, it suffices to show that the collection of maps of (2) is epimorphic when $M_{*}$ is a finitely generated and free $E_{*}$-module, since $\mathbb{T}$ commutes with filtered colimits and reflexive coequalizers. Suppose that $M_{*}$ is a finite free $E_{*}$-module. For $m \geqslant 2$ and $m \neq p$, there are two cases:

Case of $m$ relatively prime to $p$. In this case, let $r \geqslant 0$ be the largest integer such that $p^{r}<m$. I claim that

$$
\delta: \mathbb{T}_{p^{r}}\left(M_{*}\right) \otimes \mathbb{T}_{m-p^{r}}\left(M_{*}\right) \rightarrow \mathbb{T}_{m}\left(M_{*}\right)
$$

is surjective.

Case of $p \mid m$ and $m \neq p$. In this case, let $d=m / p>1$. I claim that

$$
\mu: \mathbb{T}_{d} \mathbb{T}_{p}\left(M_{*}\right) \rightarrow \mathbb{T}_{m}\left(M_{*}\right)
$$

is surjective. 
In either case, the claim proves regularity. To prove these claims, choose a finite free $E$-module $N$ such that $\pi_{*} N \approx M_{*}$. Then the claims amount to showing that

$$
L \mathbb{P}_{\Sigma_{p^{r}} \times \Sigma_{m-p^{r}}} N \rightarrow L \mathbb{P}_{m} N
$$

and

$$
L \mathbb{P}_{\Sigma_{d} \imath \Sigma_{p}} N \rightarrow L \mathbb{P}_{m} N
$$

are surjective on homotopy groups; in fact, each of these maps admits a section by (3.10), since $\Sigma_{p^{r}} \times \Sigma_{m-p^{r}} \subset \Sigma_{m}$ (in the first case) and $\Sigma_{d} \curlywedge \Sigma_{p} \subset \Sigma_{m}$ (in the second case) have index prime to $p$.

\subsection{The Frobenius class}

Let $I \subset E^{0} B \Sigma_{p^{k}}$ denote the kernel of the augmentation map $i^{*}: E^{0} B \Sigma_{p^{k}} \rightarrow E^{0}$, and let $J \subset E^{0} B \Sigma_{p^{k}}$ denote the ideal generated by the images of transfer maps from subgroups $\Sigma_{i} \times \Sigma_{p^{k}-i} \subset \Sigma_{p^{k}}$, for $0<i<p^{k}$.

Lemma 10.2. For $k>0$, the image $i^{*}(J)$ of $J$ under the augmentation map is $p E_{0}$, and $E^{0} B \Sigma_{p^{k}} /(I+J) \stackrel{\sim}{\longrightarrow} E_{0} /(p)$.

Proof. We compute the projection of the transfer ideal along the augmentation map. Let $m \geqslant 1$, and consider the fiber square

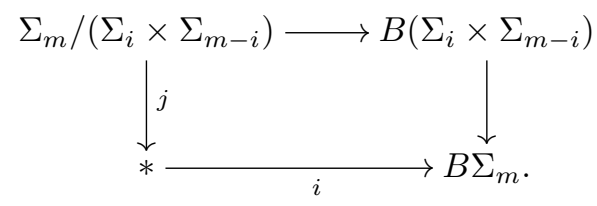

Applying the double-coset formula in E-cohomology shows that the image of

$$
E^{0} B\left(\Sigma_{i} \times \Sigma_{m-i}\right) \stackrel{\text { transfer }}{\longrightarrow} E^{0} B \Sigma_{m} \stackrel{i^{*}}{\longrightarrow} E^{0}
$$

is precisely the ideal generated by the binomial coefficient $\left(\begin{array}{c}m \\ i\end{array}\right)$. The result follows from the observation that $\operatorname{gcd}\left\{\left(\begin{array}{c}p^{k} \\ i\end{array}\right) \mid 0<i<p^{k}\right\}=p$ for $k>0$. (Note that for $m$ prime to $p, E^{0} B \Sigma_{m} / J \approx 0$ already.)

Proposition 10.3. The augmentation map $i^{*}: E^{0} B \Sigma_{p} \rightarrow E^{0}$ restricts induces an isomorphism $J \rightarrow p E^{0}$, and induces a homomorphism $\sigma^{*}: E^{0} B \Sigma_{p} / J \rightarrow E^{0} /(p)$ on quotients. Furthermore, the commutative square

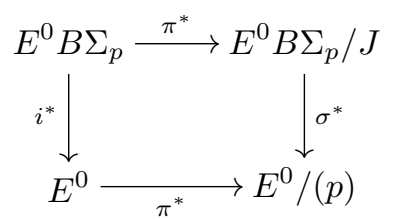

is a pullback square of $E^{0}$-modules.

Proof. Since $k=1$, the ideal $J$ is equal to the image in cohomology of the stable transfer map $t: \Sigma_{+}^{\infty} B \Sigma_{p} \rightarrow \Sigma_{+}^{\infty} B\{e\}$ associated to the inclusion of the trivial subgroup $\{e\} \subset \Sigma_{p}$. Thus $J$ is a free $E_{0}$-module on the one generator $t^{*}(1) \in \tilde{E}^{0} B \Sigma_{p}$, 
and therefore the surjective map $J \rightarrow p E_{0}$ must be an isomorphism. The existence of $\sigma^{*}$ is immediate from $(10.2)$, and the square is clearly a pullback.

For any $E_{*}$-module $M_{*}$, there are maps

$$
u: M_{*} \otimes E_{*} \Gamma[1] \rightarrow \mathbb{T}_{p}\left(M_{*}\right), \quad v: \operatorname{Sym}_{E_{*}}^{p}\left(M_{*}\right) \rightarrow \mathbb{T}_{p}\left(M_{*}\right)
$$

The map $u$ is induced by the standard $\Gamma$-module structure $\mathbb{T} M_{*} \otimes \Gamma \rightarrow \mathbb{T} M_{*}$, which carries $\mathbb{T}_{1} M_{*}$ into $\mathbb{T}_{p} M_{*}$. The map $v$ is induced by the tautological commutative $E_{*}-$ algebra map $\operatorname{Sym}_{E_{0}}^{*}\left(M_{*}\right) \rightarrow \mathbb{T}\left(M_{*}\right)$. We will be particularly be interested in the case when $M_{*}=E_{*}$, so that all objects are concentrated in even degree and the maps have the form

$$
u: \Gamma[1] \rightarrow \mathbb{T}_{p}\left(E_{0}\right), \quad v: E_{0} \rightarrow \mathbb{T}_{p}\left(E_{0}\right) .
$$

In this case, observe that $\operatorname{hom}_{E_{0}}\left(E^{0} B \Sigma_{p}, E_{0}\right) \approx \mathbb{T}_{p}\left(E_{0}\right)$, and that the inclusion

$$
u: \Gamma[1] \rightarrow \mathbb{T}_{p}\left(E_{0}\right)
$$

is dual to the projection $E^{0} B \Sigma_{p} \rightarrow E^{0} B \Sigma_{p} / I$. Likewise, observe that $v$ is dual to the augmentation map $i^{*}: E^{0} B \Sigma_{p} \rightarrow E^{0}$.

Let $\bar{\sigma} \in \Gamma[1] \otimes_{E_{0}} E_{0} / p E_{0}$ denote the element corresponding via duality to the homomorphism $\sigma^{*}: E^{0} B \Sigma_{p} / I \rightarrow E^{0} / p E^{0}$ of $(10.3)$. We will call $\bar{\sigma}$ the Frobenius class. Pick a representative $\sigma \in \Gamma[1]$ of this congruence class.

Proposition 10.4. There is a pushout square in $E_{0}$-modules of the form

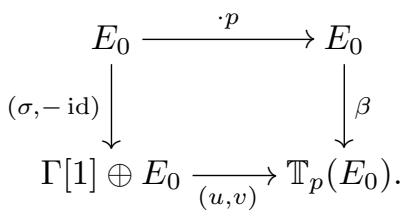

Proof. Consider the diagram

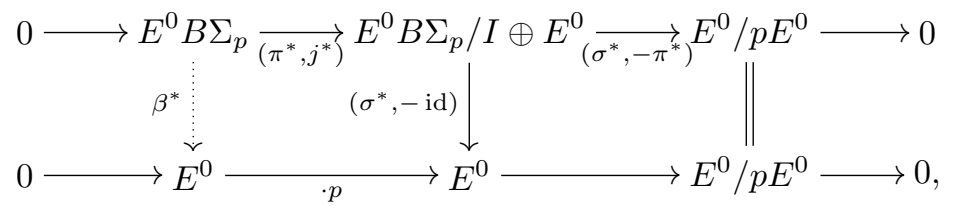

where the top row is obtained from the pullback square of $(10.3)$, and $\beta^{*}$ is the unique map making the diagram commute. The long exact sequence associated to $\operatorname{Ext}_{E^{0}}^{*}\left(-, E^{0}\right)$ gives exact sequences

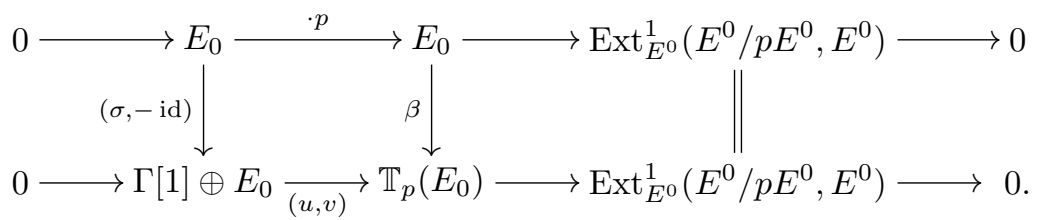

The corresponding odd degree result is simpler. 
Proposition 10.5. The inclusion $\Gamma[1] \otimes \omega^{q / 2} \rightarrow \mathbb{T}_{p}\left(\omega^{q / 2}\right)$ is an isomorphism when $q$ is odd.

Proof. By periodicity, we may assume that $q=1$. In this case, this amounts to the claim that

$$
E^{*} B \Sigma_{p}^{\rho} \rightarrow E^{*} B \Sigma_{p}^{\rho} / I
$$

is an isomorphism, where $I$ is the image of the transfer map, which is immediate from (3.13).

\subsection{The case of weight $p$}

Proposition 10.6. Let $M_{*}$ be an $E_{*}$-module. The image of the map

$$
\mathbb{T}_{p}\left(M_{*}\right) \rightarrow \mathbb{T}_{p}\left(M_{*}\right) \otimes \mathbb{Q}
$$

is generated by the images of $u, v$, and by elements of the form $\left(\sigma x-x^{p}\right) / p$ as $x$ ranges over elements of $M_{0}$.

Proof. The functors $\mathbb{T}_{p}$ and $\mathrm{Sym}^{p}$ commute with filtered colimits and reflexive coequalizers, so it suffices to check the result when $M_{*}$ is a finite free $E_{*}$-module. The "binomial formula"

$$
\mathbb{T}_{p}\left(M_{*} \oplus N_{*}\right) \approx \bigoplus_{i+j=p} \mathbb{T}_{i}\left(M_{*}\right) \otimes \mathbb{T}_{j}\left(N_{*}\right),
$$

together with the fact that $u: \operatorname{Sym}^{i}\left(M_{*}\right) \rightarrow \mathbb{T}_{i}\left(M_{*}\right)$ is an isomorphism for $i \leqslant p$, allows us to reduce to the case that $M_{*}$ is free on one generator in some degree, which amounts to (10.4) for even degree and (10.5) for odd degree.

\subsection{Proof of Theorem A}

Say an object of $\operatorname{Alg}_{\mathbb{T}}^{*}$ (resp. $\operatorname{Alg}_{\Gamma}^{*}$ ) is torsion free its underlying $E_{*}$-module is torsion free as an abelian group. Say that an object $B$ of $\operatorname{Alg}_{\Gamma}^{*}$ satisfies the congruence condition if

$$
\sigma x \equiv x^{p} \quad \bmod p B
$$

for all $x \in B^{0}$.

Let $U: \operatorname{Alg}_{\mathbb{T}}^{*} \rightarrow \operatorname{Alg}_{\Gamma}^{*}$ denote the forgetful functor. If $B \in \operatorname{Alg}_{\mathbb{T}}^{*}$, then $U(B)$ satisfies the congruence condition; this is the content of (10.4). In fact, if $x \in B^{0}$, represented by a map $f: \mathbb{T}\left(E_{0}\right) \rightarrow B$, then $\sigma x-x^{p} \in B^{0}$ is represented by the composite

$$
E_{0} \stackrel{(\sigma,-\mathrm{id})}{\longrightarrow} \Gamma[1] \oplus E_{0} \stackrel{(u, v)}{\longrightarrow} \mathbb{T}_{p}\left(E_{0}\right) \stackrel{f}{\longrightarrow} B
$$

which factors through multiplication by $p$ by (10.4).

Proof of Theorem A. By the above remarks, it suffices to show that an object $B$ of $\operatorname{Alg}_{\Gamma}^{*}$ which is torsion free and which satisfies the congruence condition admits a unique structure of $\mathbb{T}$-algebra.

Let $B^{\prime}=B \otimes \mathbb{Q}$; this is an object of $\operatorname{Alg}_{\Gamma, \mathbb{Q}}^{*}$ by $(8.2)$, and thus there is a unique $\mathbb{T}$ algebra structure on $B^{\prime}$ compatible with the $\Gamma$-algebra structure. By (9.1) and (10.1), 
it suffices to show that the dotted arrow exists in

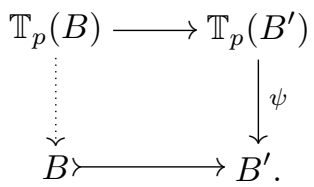

Because $B$ is a $\Gamma$-algebra, we know that $\psi(u(B \otimes \Gamma[1])) \subseteq B$ and $\psi\left(v\left(\operatorname{Sym}^{p}(B)\right)\right) \subseteq B$. The map $\mathbb{T}_{p}(B) \rightarrow B^{\prime}$ factors uniquely through a map $\mathbb{T}_{p}(B) \otimes \mathbb{Q} \rightarrow B^{\prime}$, and thus by (10.6) it suffices to check that $\psi\left(\left(\sigma x-x^{p}\right) / p\right) \in B$ for all $x \in B_{0}$; this is precisely the congruence condition.

\section{Sheaves on categories of deformations}

Fix a prime $p$ and an integer $n \geqslant 1$. Let $k$ be a perfect field of characteristic $p$, and let $G_{0}$ be a (one-dimensional, commutative) formal group of height $n$ defined over $k$.

\subsection{Deformations of $G_{0}$}

Let $\widehat{\mathcal{R}}$ denote the category whose objects are complete local rings whose residue field has characteristic $p$, and whose morphisms are continuous local homomorphisms. If $R \in \widehat{\mathcal{R}}$, then we write $\mathfrak{m} \subset R$ for the maximal ideal, and $\pi: R \rightarrow R / \mathfrak{m}$ for the quotient map.

Let $R \in \widehat{\mathcal{R}}$. A deformation of $G_{0}$ to $R$ is a triple $(G, i, \alpha)$, consisting of

(a) a formal group $G$ defined over $R$,

(b) a homomorphism $i: k \rightarrow R / \mathfrak{m}$, and

(c) an isomorphism $\alpha: i^{*} G_{0} \rightarrow \pi^{*} G$ of formal groups over $R / \mathfrak{m}$.

\subsection{The Frobenius isogeny}

Suppose that $R \in \widehat{\mathcal{R}}$ has characteristic $p$. We write $\phi: R \rightarrow R$ for the $p$ th power homomorphism $\left(\phi(x)=x^{p}\right)$. For each formal group $G$ over such a ring $R$, the Frobenius isogeny Frob: $G \rightarrow \phi^{*} G$ is the homomorphism of formal groups over $R$ induced by the relative Frobenius map on rings. We write Frob ${ }^{r}: G \rightarrow\left(\phi^{r}\right)^{*} G$ for the homomorphism inductively defined by Frob $^{r}=\phi^{*}\left(\right.$ Frob $\left.^{r-1}\right) \circ$ Frob.

\subsection{The "deformations of Frobenius" category}

Let $(G, i, \alpha)$ and $\left(G^{\prime}, i^{\prime}, \alpha^{\prime}\right)$ be two deformations of $G_{0}$ to $R$. We say that a homomorphism $f: G \rightarrow G^{\prime}$ of formal groups over $R$ is a deformation of Frob $^{r}$ if

(i) $i \circ \phi^{r}=i^{\prime}$, so that $\left(\phi^{r}\right)^{*} i^{*} G_{0} \approx\left(i^{\prime}\right)^{*} G_{0}$, and

(ii) the square

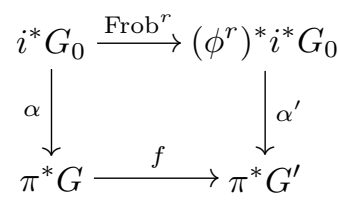

of homomorphisms of formal groups over $R / \mathfrak{m}$ commutes. 
We let $\operatorname{Def}_{R}$ denote the category whose objects are deformations of $G_{0}$ to $R$, and whose morphisms are homomorphisms which are a deformation of Frob ${ }^{r}$ for some (unique) $r \geqslant 0$.

Remark 11.1. Say that a morphism in $\operatorname{Def}_{R}$ has a height $r$ (where $r \geqslant 0$ ), if it is a deformation of $\mathrm{Frob}^{r}$. The height of a composition of morphisms is the sum of the heights. For $G, G^{\prime}$ objects of $\operatorname{Def}_{R}$, let $\operatorname{Def}_{R}^{r}\left(G, G^{\prime}\right) \subset \operatorname{Def}_{R}\left(G, G^{\prime}\right)$ denote the subset of the hom-set consisting of morphisms of height $r$. Note that $\operatorname{Def}_{R}^{0}\left(G, G^{\prime}\right)$ are precisely the isomorphisms from $G$ to $G^{\prime}$ in Def, i.e., the isomorphisms $G \rightarrow G^{\prime}$ of formal groups which are deformations of the identity map of $G_{0}$.

Observe that if $R$ is an $\mathbb{F}_{p}$-algebra, then for every object $(G, i, \alpha)$ in $\operatorname{Def}_{R}$ the Frobenius isogeny defines a morphism Frob: $(G, i, \alpha) \rightarrow\left(\phi^{*} G, \phi i, \phi^{*} \alpha\right)$ in this category.

Given $f: R \rightarrow R^{\prime} \in \widehat{\mathcal{R}}$, we define a functor $f^{*}: \operatorname{Def}_{R^{\prime}} \rightarrow \operatorname{Def}_{R}$ by base change. The base change functors are coherent, in the sense that if $R \stackrel{f}{\rightarrow} R^{\prime} \stackrel{g}{\rightarrow} R^{\prime \prime}$, then there are natural isomorphisms $g^{*} f^{*} \approx(g f)^{*}$ satisfying the evident coherence property.

Remark 11.2. We can summarize the above using 2-categorical language: Def is a pseudofunctor from $\widehat{\mathcal{R}}$ to the 2-category grCat of graded categories.

\subsection{Lubin-Tate theory}

According to the deformation theory of Lubin-Tate, there exists a ring $L \in \widehat{\mathcal{R}}$, and a deformation $\left(G_{\text {univ }}\right.$, id, $\left.\alpha_{\text {univ }}\right)$ which is a deformation of $G_{0}$ to $L$, such that for each $G \in \operatorname{Def}_{R}$ there exists a unique map $f: L \rightarrow R \in \widehat{\mathcal{R}}$ for which there is an isomorphism $u: G \rightarrow f^{*} G_{\text {univ }}$ in $\operatorname{Def}_{R}$, and that furthermore the isomorphism $u$ is itself unique. The ring $L$ has the form $L \approx \mathbb{W} k \llbracket u_{1}, \ldots, u_{n-1} \rrbracket$. As we hope the reader is already aware, $L$ is canonically identified with $\pi_{0} E$ for the Morava $E$-theory spectrum associated to $G_{0}$.

Say that a groupoid $D$ is thin if between any two objects of $D$ there is at most one isomorphism. As a consequence of the Lubin-Tate theorem, the groupoids $\operatorname{Def}_{R}^{0}$ are thin.

Given a category $C$ and a subcategory $D \subseteq C$ which is a thin groupoid and which contains all the objects of $C$, we can define a quotient category $C / D$ as follows. The object set $\mathrm{ob}(C / D)$ is defined to be ob $C / \sim$, where we say that $X \sim Y$ if there exists $f: X \rightarrow Y \in D$; morphisms in $C / D$ are defined by $(C / D)([X],[Y])=C(X, Y)$. That this becomes a category uses the fact that $D$ is thin. There is an evident quotient functor $C \rightarrow C / D$, which is an equivalence of categories.

\subsection{Subgroups of formal groups}

Let $\operatorname{Sub}_{R} \stackrel{\text { def }}{=} \operatorname{Def}_{R} / \operatorname{Def}_{R}^{0}$. Thus the quotient functors $\operatorname{Def}_{R} \rightarrow \operatorname{Sub}_{R}$ are equivalences of categories for all $R$. The assignment $R \mapsto \operatorname{Sub}_{R}$ is a functor $\widehat{\mathcal{R}} \rightarrow$ Cat, (i.e., not merely a pseudofunctor.)

Proposition 11.3. There is a one-to-one correspondence ob $\operatorname{Sub}_{R} \approx \widehat{\mathcal{R}}(L, R)$.

Proof. This is Lubin-Tate deformation theory. 
For objects $G, G^{\prime} \in \operatorname{Sub}_{R}$, let $\operatorname{Sub}_{R}^{r}\left(G, G^{\prime}\right) \subset \operatorname{Sub}_{R}\left(G, G^{\prime}\right)$ denote the image of $\operatorname{Def}_{R}^{r}\left(G, G^{\prime}\right)$.

Proposition 11.4. Given a deformation $G$, the assignment $f \mapsto \operatorname{ker} f$ is a one-toone correspondence between $\coprod_{\left[G^{\prime}\right] \in \mathrm{obSub}_{R}} \operatorname{Sub}_{R}^{r}\left(G, G^{\prime}\right)$ and the set of finite subgroup schemes of $G$ which have rank $p^{r}$.

Thus any pair $([G], K)$ consisting of an object $[G]$ of $\operatorname{Sub}_{R}$ and a subgroup $K$ of $G$ of rank $p^{r}$ determines an object $\left[G^{\prime}\right]$ of $\mathrm{Sub}_{R}$, which is represented by any deformation of Frobenius $f: G \rightarrow G^{\prime}$ with $\operatorname{ker} f=K$; we will write $[G / K]$ for the object $\left[G^{\prime}\right]$.

Proposition 11.5. For each $r \geqslant 0$ there is a complete local ring $L[r]$ such that $\widehat{\mathcal{R}}(L[r], R)$ is in one-to-one correspondence with the set of pairs $([G], K)$, where $[G]$ is an object of $\operatorname{Sub}_{R}$ and $K$ is a subgroup of $G$ of degree $p^{r}$. Under the continuous homomorphism $L \rightarrow L[r]$ which classifies $([G], K) \mapsto[G]$, the ring $L[r]$ is finite and free as an L-module.

Proof. See [Str97].

Corollary 11.6. Let

$$
\operatorname{Sub}_{R}^{r}=\coprod_{[G],\left[G^{\prime}\right] \in \mathrm{ob} \mathrm{Sub}_{R}} \operatorname{Sub}_{R}^{r}\left(G, G^{\prime}\right) .
$$

Then $\operatorname{Sub}_{R}^{r} \approx \widehat{\mathcal{R}}(L[r], R)$.

\subsection{The formal graded category scheme Sub}

Let can* $: L[r] \rightarrow E_{0} /(p)$ be the map that classifies the kernel of Frob ${ }^{r}: \pi^{*} G_{\text {univ }} \rightarrow$ $\left(\phi^{r}\right)^{*} \pi^{*} G_{\text {univ }}$, where $\pi: E_{0} \rightarrow E_{0} /(p)$. Observe that if a deformation $g$ of Frob $^{r}$ is represented by some ring map $f: L[r] \rightarrow R$, then $f$ factors through can* if and only if $g$ is equal to Frob $^{r}$.

Let $\operatorname{can}_{0}^{*}: L[r] \rightarrow k$ denote the composite of can* with $E_{0} /(p) \rightarrow k$; it classifies the kernel of Frob $^{r}: G_{0} \rightarrow\left(\phi^{r}\right)^{*} G_{0}$. The maps can* and can $_{0}^{*}$ are surjective, and the kernel of $\operatorname{can}_{0}^{*}$ is precisely the maximal ideal of $L[r]$. Let $s^{*}, t^{*}: L[0] \rightarrow L[r]$ be the maps in $\widehat{\mathcal{R}}$ which classify $([G], K) \mapsto[G]$ and $([G], K) \mapsto[G / K]$ respectively. It is clear that the diagram

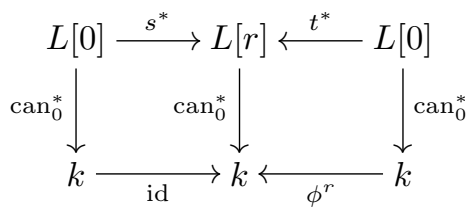

commutes. Since $k$ is perfect, we see that the tensor product $L[r]_{s} \otimes_{E_{0}} L\left[r^{\prime}\right]$ is also a complete local ring with residue field $k$. There are maps $c^{*}: L\left[r+r^{\prime}\right] \rightarrow L[r]_{s} \otimes_{E_{0}}$ ${ }_{t} L\left[r^{\prime}\right]$ and $i^{*}: L[0] \rightarrow E_{0}$ in $\widehat{\mathcal{R}}$, corresponding to composition and identity maps in Sub; from what we have already shown, it follows that $c^{*}$ and $i^{*}$ are local homomorphisms, inducing isomorphisms on the residue fields. In fact, $i^{*}$ is an isomorphism.

Thus, Sub is what we might call a formal affine category scheme; it is represented by data $\mathcal{L}=\left(E_{0}, L[r], s^{*}, t^{*}, i^{*}, c^{*}\right)$, which are objects and morphisms in $\widehat{\mathcal{R}}$. 


\subsection{Sheaves on the deformations category}

For $R \in \widehat{\mathcal{R}}$, let $\operatorname{Mod}_{R}$ and $\mathrm{Alg}_{R}$ denote the category of $R$-modules and commutative $R$-algebras respectively. If $f: R \rightarrow R^{\prime} \in \widehat{\mathcal{R}}$, let $f^{*}: \operatorname{Mod}_{R} \rightarrow \operatorname{Mod}_{R^{\prime}}\left(\right.$ or $f^{*}: \operatorname{Alg}_{R} \rightarrow$ $\left.\operatorname{Alg}_{R^{\prime}}\right)$ denote the evident basechange functors, defined in either case by $f^{*}(M) \approx$ $R^{\prime} \otimes_{R} M$. These functors are coherent, in the sense that if $R \stackrel{f}{\rightarrow} R^{\prime} \stackrel{g}{\rightarrow} R^{\prime \prime}$ then there are natural isomorphisms $g^{*} f^{*} \approx(g f)^{*}$ satisfying the evident coherence property.

A quasi-coherent sheaf of $\mathcal{O}$-modules on Def consists of data of the form $M=\left(\left\{M_{R}\right\},\left\{M_{f}\right\}\right)$, where for each $R \in \widehat{\mathcal{R}}$ we have a functor $M_{R}: \operatorname{Def}_{R}^{\text {op }} \rightarrow \operatorname{Mod}_{R}$, and for each $f: R \rightarrow R^{\prime} \in \widehat{\mathcal{R}}$, we have a natural isomorphism $M_{f}: f^{*} M_{R} \stackrel{\sim}{\longrightarrow} M_{R^{\prime}} f^{*}$,

such that for all $R \stackrel{f}{\rightarrow} R^{\prime} \stackrel{g}{\rightarrow} R^{\prime \prime}$, both ways of constructing a natural isomorphism $g^{*} f^{*} M_{R} \rightarrow M_{R^{\prime}} g^{*} f^{*}$ coincide, and for $\operatorname{id}_{R}: R \rightarrow R$, the natural isomorphism $M_{\mathrm{id}_{R}}$ : $\operatorname{id}_{R}^{*} M_{R} \rightarrow M_{R}$ id $_{R}^{*}$ is the identity transformation.

A morphism $\gamma: M \rightarrow N$ of quasi-coherent sheaves modules consists of data $\left\{\gamma_{R}\right\}$, where $\gamma_{R}: M_{R} \rightarrow N_{R}$ for each $R \in \widehat{\mathcal{R}}$ is a natural transformation compatible with base change, in the sense that if $f: R \rightarrow R^{\prime}$ in $\widehat{\mathcal{R}}$, then $N_{f}\left(f^{*} \gamma_{R}\right)=\left(\gamma_{R} f^{*}\right) M_{f}$.

Let $\mathrm{Sh}$ (Def, Mod) denote the category of quasi-coherent sheaves of $\mathcal{O}$-modules. It is a tensor category, with tensor product defined "objectwise", so that $(M \otimes N)_{R}(G)=$ $M_{R}(G) \otimes_{R} N_{R}(G)$. The unit object is $\mathcal{O}$, defined so that $\mathcal{O}_{R}: \operatorname{Def}_{R} \rightarrow \operatorname{Mod}_{R}$ is the constant functor sending every object to $R$.

In a similar way, we define a category $\mathrm{Sh}(\mathrm{Def}, \mathrm{Alg})$ of quasi-coherent sheaves of $\mathcal{O}$-algebras, by replacing " $\operatorname{Mod}_{R}$ " in the above definitions with " $\operatorname{Alg}_{R}$ ". The category $\mathrm{Sh}$ (Def, Alg) is plainly isomorphic to the category of commutative monoid objects in $\operatorname{Sh}($ Def, Mod).

It is clear from the definition that $\mathrm{Sh}(\mathrm{Def}, \mathrm{Alg})$ and $\mathrm{Sh}$ (Def, Mod) have all small colimits and finite products.

Remark 11.7. The above is easily summarized using 2-categorical language: Def, Mod, and Alg are pseudofunctors $\widehat{\mathcal{R}} \rightarrow$ Cat, and Sh(Def, Mod) and Sh(Def, Alg) are defined to be the categories of pseudonatural transformations and modifications between pseudofunctors Def $\rightarrow$ Mod and Def $\rightarrow$ Alg respectively.

\subsection{Sheaves are comodules}

Recall that for a graded affine category scheme such as defined by the data $\mathcal{L}$, there is an associated category of comodules $\operatorname{Comod}_{\mathcal{L}}(5.5)$.

Proposition 11.8. There is an equivalence of tensor categories between

$$
\operatorname{Sh}\left(\text { Def, Mod) and } \operatorname{Comod}_{\mathcal{L}} .\right.
$$

Proof. This is straightforward. Given an $\mathcal{L}$-comodule $(M, \psi: M \rightarrow V M)$, we produce a sheaf by the following procedure. Let

$$
\tilde{\psi}_{r}: L[r]_{t} \otimes_{E_{0}} M \rightarrow L[r]_{s} \otimes_{E_{0}} M
$$

denote the extension of $\psi_{r}: M \rightarrow V M \rightarrow L[r]_{s} \otimes_{E_{0}} M$ to a map of $L[r]$-modules. 
On objects $h: E_{0} \rightarrow R$ of $\operatorname{Def}_{R}$, the functor $M_{R}: \operatorname{Def}_{R} \rightarrow \operatorname{Mod}_{R}$ is given on objects of $\operatorname{Def}_{R}$ by

$$
M_{R}(h)=R_{h} \otimes_{E_{0}} M
$$

On morphisms $g: L[r] \rightarrow R$ of Pow $_{R}$, the functor $M_{R}$ is given by

$$
\begin{aligned}
M_{R}(g: L[r] \rightarrow R) & = \\
& \left(R_{t^{*} g} \underset{E_{0}}{\otimes} M \approx R_{g} \underset{L[r]}{\otimes} L[r]_{t} \underset{E_{0}}{\otimes} M \stackrel{\text { id } \tilde{\otimes} \psi_{r}}{\longrightarrow} R_{g} \underset{L[r]}{\otimes} L[r]_{s} \underset{E_{0}}{\otimes} M \approx R_{s^{*} g} \underset{E_{0}}{\otimes} M\right) .
\end{aligned}
$$

Likewise, given an object $M$ of $\operatorname{Sh}($ Def, Mod), we obtain an $\mathcal{L}$-comodule by evaluating $M$ at the the universal deformation of $G_{0}$, and at the universal examples of deformations of $\mathrm{Frob}^{r}$.

(Note that although $\mathcal{L}$ is really a formal graded category scheme, formality does not play a role here.)

\subsection{The sheaves $\mathcal{F}, \omega$, and $\mathbb{Z} / 2$-graded sheaves}

If $G$ is a formal group over a local ring $R$, then let $F_{G}$ denote the ring of functions on $G$; it is an $R$-algebra non-canonically isomorphic to $R \llbracket x \rrbracket$. We define $\mathcal{F} \in \operatorname{Sh}($ Def, Alg) by

$$
\mathcal{F}_{R}((G, i, \alpha)) \stackrel{\text { def }}{=} F_{G} .
$$

The evident algebra maps $\mathcal{O} \rightarrow \mathcal{F} \stackrel{e}{\rightarrow} \mathcal{O}$ induce a direct sum splitting $\mathcal{F} \approx \mathcal{O} \oplus \mathcal{I}$ as modules. Let

$$
\omega \stackrel{\text { def }}{=} \mathcal{I} / \mathcal{I}^{2}
$$

as an object of $\operatorname{Sh}\left(\right.$ Def, Mod); observe that the $R$-modules $\omega_{R}((G, i, \alpha))$ are noncanonically isomorphic to $R$, so that $\omega$ is a symmetric object in the sense of $\S 2.1$.

Let $\mathrm{Sh}(\mathrm{Def}, \mathrm{Mod})^{*}$ denote the $\mathbb{Z} / 2$-graded $\omega$-twisted tensor category ( $\left.\S 2\right)$ associated to $\mathrm{Sh}(\mathrm{Def}, \mathrm{Mod})$. Let $\mathrm{Sh}(\mathrm{Def}, \mathrm{Alg})^{*}$ denote the category of commutative monoid objects in $\operatorname{Sh}($ Def, Mod)*.

\subsection{Frobenius congruence}

Suppose that $R \in \widehat{\mathcal{R}}$ has characteristic $p$. The $p$ th power map $\phi$ defines a functor $\phi^{*}: \operatorname{Alg}_{R} \rightarrow \operatorname{Alg}_{R}$ by base change, together with a natural transformation Frob: $\phi^{*} \rightarrow$ id, where Frob: $\phi^{*} A \rightarrow A$ is the relative Frobenius defined as the unique map making the diagram

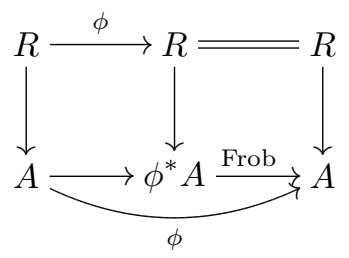

commute. 
Given an object $B \in \mathrm{Sh}$ (Def, Alg), we say it satisfies the Frobenius congruence if for all $R \in \widehat{\mathcal{R}}$ of characteristic $p$, and for all $G \in \operatorname{Def}_{R}$, the diagram

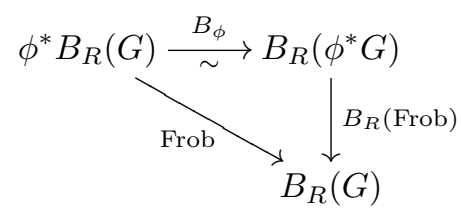

commutes. Roughly speaking, the Frobenius congruence condition says that $B$ carries the relative Frobenius on formal groups to the relative Frobenius on algebras.

Example 11.9. Both $\mathcal{O}$ and $\mathcal{F}$ satisfy the Frobenius congruence.

Let $\mathrm{Sh}(\mathrm{Def}, \mathrm{Alg})_{\text {cong }} \subset \mathrm{Sh}(\mathrm{Def}, \mathrm{Alg})$ denote the full subcategory consisting of sheaves which satisfy the Frobenius congruence. Let Sh(Def, Alg) cong $^{*} \subset \mathrm{Sh}(\mathrm{Def}, \mathrm{Alg})^{*}$ denote the full subcategory consisting of sheaves whose even degree part is in $\mathrm{Sh}(\text { Def, } \mathrm{Alg})_{\text {cong. }}$.

Proposition 11.10. Let $A$ be an object of $\mathrm{Sh}(\operatorname{Def}, \mathrm{Alg})$, and let $(B, \psi: B \rightarrow C B)$ be the corresponding commutative monoid object in $\operatorname{Comod}_{\mathcal{A}}$. Then A satisfies the Frobenius congruence if and only if the composite map

$$
B \stackrel{\psi_{1}}{\longrightarrow} L[1]_{s} \otimes_{E_{0}} B \stackrel{\text { can }^{*} \otimes \mathrm{id}}{\longrightarrow}\left(E_{0} /(p)\right)_{s} \otimes_{E_{0}} B \approx B / p B
$$

is equal to $x \mapsto x^{p}$.

Proof. This amounts to checking the congruence condition on the universal example.

\section{Bialgebra rings as sheaves on the deformation category}

We retain the notation of the previous section.

\subsection{The formal graded category scheme Pow}

The discussion of $\S 5.5$ applies to our twisted $L$-bialgebra $\Gamma$ of power operations defined in $\S 6$. Thus, let $\mathcal{A}=\left(E_{0}, A[r], s^{*}, t^{*}, i^{*}, c^{*}\right)$ be the data obtained by "dualizing" $\Gamma$, so that $A[r] \approx H_{\Gamma[r]}\left(E_{0}\right) \approx E^{0} B \Sigma_{p^{r}} /$ (transfer), and in particular $A[0] \approx E_{0}$. Our first order of business is to show that, like $\mathcal{L}$, the data $\mathcal{A}$ consists of objects and morphisms of $\widehat{\mathcal{R}}$, and thus defines a formal graded category scheme (11.6), which we will call Pow.

For $r>0$, let $\bar{A}[r]$ denote the quotient ring of $A[r]$ defined by

$$
\bar{A}[r] \stackrel{\text { def }}{=} E^{0} B \Sigma_{p^{r}} /((\text { transfer })+(\text { aug })),
$$

where (aug) denotes the kernel of the augmentation map $E^{0} B \Sigma_{p^{r}} \rightarrow E^{0}$ induced by inclusion of the base point $* \rightarrow B \Sigma^{p^{r}}$. Additionally, we set $\bar{A}[0] \stackrel{\text { def }}{=} E_{0} / p E_{0}$. By (10.2), the augmentation quotient induces an isomorphism $\bar{A}[r] \rightarrow E_{0} /(p)$.

Lemma 12.1. The rings $A[r]$ are local rings, with maximal ideal given by the kernel of the composite $A[r] \rightarrow \bar{A}[r] \approx E_{0} / p E_{0} \rightarrow k$. 
Proof. The ring $A[r]$ is finite as an $E_{0}$-module (using $s^{*}$ ), and thus every maximal ideal of $A[r]$ must contract to the maximal ideal of $E_{0}$ by the "going up theorem". Thus it suffices to show that $A[r] \otimes_{E_{0}} k$ is a local ring. Since $A[r]$ is a quotient of $E^{0} B \Sigma_{p^{r}}$, it suffices to show that $E^{0} B \Sigma_{p^{r}} \otimes_{E_{0}} k$ is a local ring. Let $K$ denote the generalized cohomology theory obtained by killing the maximal ideal of $E$, so that $E^{0} B \Sigma_{p^{r}} \otimes_{E_{0}} k \approx K^{0} B \Sigma_{p^{r}}$; the result follows from the observation that the augmentation ideal of $K^{0} B \Sigma_{p^{r}}$ is nilpotent, since $B \Sigma_{p^{r}}$ is connected of finite type.

Lemma 12.2. Let $B$ be an object in $\mathrm{Alg}_{\mathbb{T}}$. Then the composite

$$
B_{0} \rightarrow \operatorname{Hom}_{E_{*}}(\Gamma[r], B) \approx B \otimes_{E_{0} s} A[r] \rightarrow B \otimes_{E_{0} s} \bar{A}[r] \approx B / p B
$$

sends $x \mapsto x^{p^{r}}$.

Proof. Immediate using (4.11) and (10.2).

Let can $^{*}: A[r] \rightarrow \bar{A}[r] \approx k$ denote the evident projection. The diagram

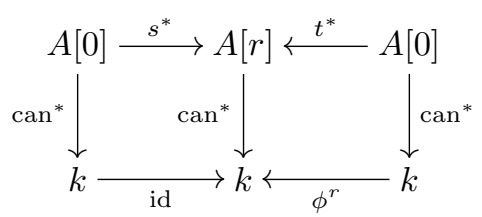

commutes, because $s^{*}$ is induced by the "standard" inclusion $E_{0} \rightarrow E^{0} B \Sigma_{p^{k}}$, while $t^{*}$ is induced by the structure map $E_{0} \rightarrow \operatorname{Hom}_{E_{0}}\left(\Gamma[r], E_{0}\right)$ which defines the canonical $\Gamma$-module structure on the ground ring $E_{0}$, and thus is the $p^{r}$ th power map on quotients by (12.2). Thus the rings $A[r]_{s} \otimes_{A[0]} A\left[r^{\prime}\right]$ are complete local with residue field $k$, and the homomorphisms $s^{*}, t^{*}, c^{*}, i^{*}$ are all local homomorphisms.

Thus, Pow is a formal graded category scheme. Observe that $\operatorname{Pow}_{R}^{0}$ is a thin groupoid for all $R \in \widehat{\mathcal{R}}$, and that Pow : $\widehat{\mathcal{R}} \rightarrow$ grCat is a functor (not just a pseudofunctor).

\subsection{Isomorphism between Sub and Pow}

We are going to construct a pseudonatural transformation $F:$ Pow $\rightarrow$ Def of pseudofunctors $\widehat{\mathcal{R}} \rightarrow$ grCat, and we will show, by applying a theorem of Strickland, that $F_{R}:$ Pow $_{R} \rightarrow \operatorname{Def}_{R}$ is an equivalence of graded categories for each $R \in \widehat{\mathcal{R}}$. This immediately implies the following.

Proposition 12.3. The natural transformation

$$
F^{\prime}: \text { Pow } \stackrel{F}{\longrightarrow} \text { Def } \rightarrow \text { Sub }
$$

of functors $\widehat{\mathcal{R}} \rightarrow$ grCat induces an isomorphism $\mathrm{Pow}_{R} \rightarrow \mathrm{Sub}_{R}$ of graded categories for all $R$ in $\widehat{\mathcal{R}}$.

Observe that an object $M \in \operatorname{Mod}_{\Gamma}$ determines functors $M_{R}: \operatorname{Pow}_{R} \rightarrow \operatorname{Mod}_{R}$, as follows. Let $\psi: M \rightarrow H_{\Gamma[r]}(M) \approx A[r]_{s} \otimes_{E_{0}} M$ denote the structure map of the $\Gamma$-module $M$; it is a map of $E_{0}$-modules, using the module structure on $A[r]$ given by the ring homomorphism $t^{*}: E_{0} \rightarrow A[r]$. If $X$ is a space, then $E^{0} X$ is an object of $\operatorname{Alg}_{\Gamma}$, and thus we get a functor $\left(E^{0} X\right)_{R}: \operatorname{Pow}_{R} \rightarrow \operatorname{Alg}(R)$. 
The next observation is that if we take $X=\mathbb{C P}^{\infty}$, then we get a functor from Pow ${ }_{R}$ to $\operatorname{Def}_{R}$. More precisely, for each $f: E_{0} \rightarrow R$, the projective system $\left\{R \otimes_{E_{0}} E^{0} \mathbb{C P}^{m}\right\}$ determines a formal scheme over $R$, and the maps

$$
\left\{R \otimes_{E_{0}} E^{0} \mathbb{C P}^{i+j} \rightarrow R \otimes_{E_{0}} E^{0}\left(\mathbb{C P}^{i} \times \mathbb{C P}^{j}\right)\right\}
$$

give it the structure of a formal group over $R$, which we will call $f^{*} G_{\text {univ }}$. Let $f_{0}: k \rightarrow$ $R / \mathfrak{m}$ be the homomorphism obtained from $f$ by passing to residue fields. Then the triple $F(f)=\left(f^{*} G_{\text {univ }}, f_{0}\right.$, id : $\left.f_{0}^{*} G_{0} \rightarrow \pi^{*} f^{*} G_{\text {univ }}\right)$ is a deformation of $G_{0}$ to $R$.

A map $g: A[r] \rightarrow R$ determines a homomorphism of formal groups $\left(s^{*} g\right)^{*} G_{\text {univ }} \rightarrow$ $\left(t^{*} g\right)^{*} G_{\text {univ }}$ over $R$.

Lemma 12.4. This homomorphism is a deformation of Frob $^{r}$; i.e., it determines a morphism $F\left(s^{*} g\right) \rightarrow F\left(t^{*} g\right)$ in $\operatorname{Def}_{R}^{r}$.

Proof. It suffices to check the universal example, corresponding to the identity map of $A[r]$. In this case, it is enough to note that the composite

$$
E^{0} \mathbb{C P}^{\infty} \stackrel{\psi}{\rightarrow} H_{\Gamma[k]}\left(E^{0} \mathbb{C P}^{\infty}\right) \rightarrow E^{0} \mathbb{C P}^{\infty} \otimes_{E_{0}} E_{0} /(p) \rightarrow E^{0} \mathbb{C P}^{\infty} \otimes_{E_{0}} k
$$

is the map $x \mapsto x^{p^{r}}$, and that this composite is the same as

$$
E^{0} \mathbb{C P}^{\infty} \rightarrow A[r]_{s} \otimes_{E_{0}} E^{0} \mathbb{C P}^{\infty} \rightarrow \bar{A}[r]_{s} \otimes_{E_{0}} E^{0} \mathbb{C P}^{\infty}
$$

Thus we have obtained functors $F: \operatorname{Pow}_{R} \rightarrow \operatorname{Def}_{R}$, which are clearly natural in $R$, and thus a functor $F^{\prime}: \operatorname{Pow}_{R} \rightarrow \operatorname{Sub}_{R}$. By the Yoneda lemma, the functor $F^{\prime}$ determines and is determined by a collection of homomorphisms $F^{*}: L[r] \rightarrow A[r]$ in $\widehat{\mathcal{R}}$.

It remains to show that $F^{\prime}$ is an isomorphism of graded categories. It is clear that $F^{\prime}$ is a bijection on objects. To show that $F$ is a bijection on morphism, it suffices to check that $F^{*}: L[r] \rightarrow A[r]$ is an isomorphism for each $r \geqslant 0$. By the definition of $L[r]$, this map classifies a pair $([G], K)$, where $G=F\left(s^{*}: E_{0} \rightarrow A[r]\right)$ and $K$ is the kernel of the isogeny $F\left(\operatorname{id}_{A[r]}\right): F\left(s^{*}: E_{0} \rightarrow A[r]\right) \rightarrow F\left(t^{*}: E_{0} \rightarrow A[r]\right)$. The kernel $K$ is a closed subscheme of $G$; its function $\operatorname{ring} \mathcal{O}_{K}$ is the pushout of $E_{0}$-algebras

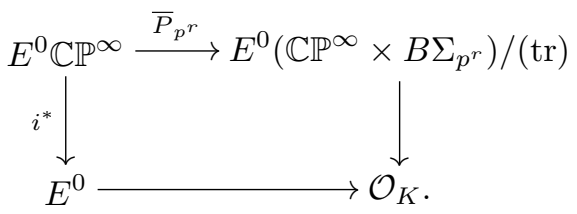

Lemma 12.5. Let $\rho_{p^{r}}^{\mathbb{C}}$ denote the complex vector bundle over $B \Sigma_{p^{r}}$ associated to the permutation representation, and let $\pi: \mathbb{P}\left(\rho_{p^{r}} \otimes \mathbb{C}\right) \rightarrow B \Sigma_{p^{r}}$ denote the projective bundle associated to this vector bundle, with tautological line bundle $L$ classified by $\ell: \mathbb{P}\left(\rho_{p^{r}}^{\mathbb{C}}\right) \rightarrow \mathbb{C P}^{\infty}$. Then there is a pushout square of $E_{0}$-algebras of the form

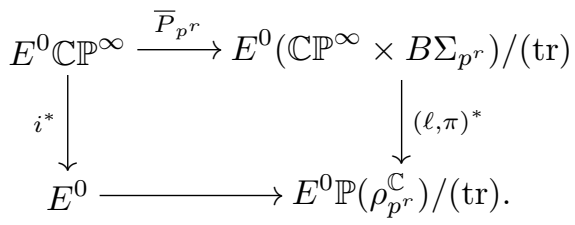


Proof. Examine the diagram

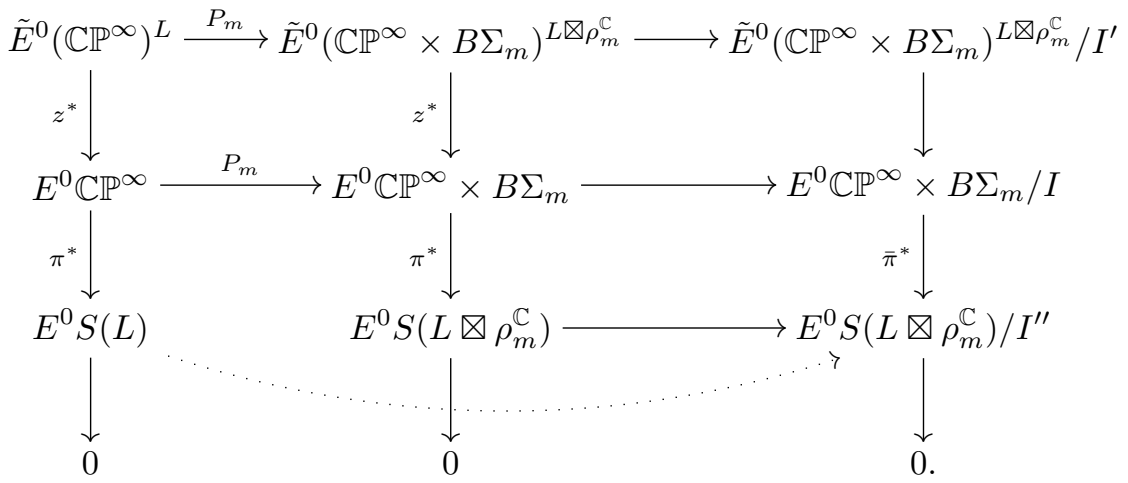

The left-hand and middle columns of vertical maps of (3) arise from the cofiber sequence coming from the pair $(D(V), S(V))$ associated to the Thom space $X^{V}$; they are exact, since the Euler class of any complex bundle of the form $L \otimes V \rightarrow \mathbb{C P}^{\infty} \times X$ is a non-zero divisor. The right-hand vertical column is obtained by quotienting the middle column by transfer ideals, denoted $I, I^{\prime}$, and $I^{\prime \prime}$. We claim that the right-hand column of (3) is also exact, which amounts to showing that $\pi^{*} I=I^{\prime \prime}$, which in turns amounts to the observation that the diagrams

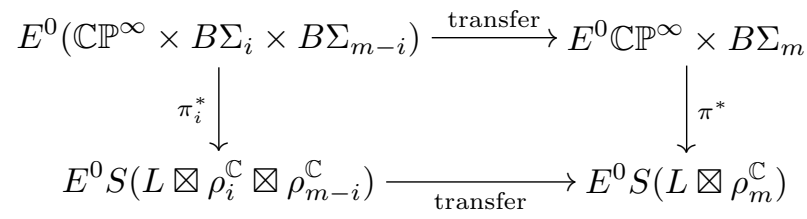

commute, and that the map marked $\pi_{i}^{*}$ is also surjective. (Note that if $m$ is not a $p$ th power, then the right-hand column of (3) is identically 0. )

The two long horizontal composites in (3) are ring homomorphisms, which we denote $\bar{P}_{m}$.

Choose any complex orientation for $E$, let $u \in \tilde{E}^{0}\left(\mathbb{C P}^{\infty}\right)^{L}$ denote the Thom class of $L$, and let $x=z^{*}(u) \in E^{0} \mathbb{C P}^{\infty}$ denote the associated Euler class, so that $E^{0} S(L) \approx$ $E^{0} \mathbb{C} \mathbb{P}^{\infty} /(x)$.

We have that $i^{*} P_{m}(x)=i^{*} z^{*} P_{m}(u)=0$, and therefore there is a ring homomorphism indicated by the dotted arrow in (3) making the square

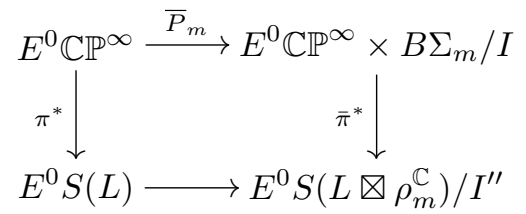

commute. Since the class $P_{m}(u)$ is a Thom class for $L \otimes \rho_{m}^{\mathbb{C}}$, the kernel of $\pi^{*}$ is a cyclic ideal generated by $P_{m}(x)=z^{*} P_{m}(u)$, and thus the kernel of $\bar{\pi}^{*}$ is also a cyclic ideal generated by $\bar{P}_{m}(x)$. Therefore we conclude that the square is a pushout square in rings; since $S(L) \approx *$ and $S\left(L \otimes \rho_{m}^{\mathbb{C}}\right) \approx \mathbb{P}\left(\rho_{m}^{\mathbb{C}}\right)$, we obtain the desired result. 
Theorem 12.6 (Strickland, [Str98]). The map $L[r] \rightarrow A[r] \approx E^{0} B \Sigma_{p^{r}} /(t r)$ classifying the subgroup $K$ of $G$ described above is an isomorphism.

As an immediate corollary, the maps $F^{*}: L[r] \rightarrow A[r]$ are isomorphisms as desired.

12.3. Equivalence of $\mathrm{Sh}(\mathrm{Def}, \mathrm{Mod})$ and $\operatorname{Mod}_{\Gamma}$, and proof of Theorem B

We have equivalences of tensor categories

$$
\operatorname{Sh}(\text { Def, } \operatorname{Mod}) \approx \operatorname{Comod}_{\mathcal{L}} \approx \operatorname{Comod}_{\mathcal{A}} \approx \operatorname{Mod}_{\Gamma},
$$

which clearly induce an equivalence $\operatorname{Sh}(\operatorname{Def}, \mathrm{Alg}) \approx \operatorname{Alg}_{\Gamma}$. The standard object $\omega$ of $\mathrm{Sh}$ (Def, Mod) defined above corresponds to the $\Gamma$-module $\omega$ defined above, and thus we obtain an equivalence of tensor categories $\mathrm{Sh}(\mathrm{Def}, \mathrm{Alg})^{*} \approx \mathrm{Alg}_{\Gamma}^{*}$. Theorem B follows immediately, with the statement about congruence conditions proved using (11.10).

\section{References}

[AHS04] M. Ando, M.J. Hopkins, and N.P. Strickland, The sigma orientation is an $H_{\infty}$ map, Amer. J. Math. 126 (2004), no. 2, 247-334. MR 2045503 (2005d:55009)

[BW05] J. Borger and B. Wieland, Plethystic algebra, Adv. Math. 194 (2005), no. 2, 246-283. MR 2139914 (2006i:13044)

[Bou96] A.K. Bousfield, On $\lambda$-rings and the $K$-theory of infinite loop spaces, $K$ Theory 10 (1996), no. 1, 1-30. MR 1373816 (98a:55006)

[BMMS86] R.R. Bruner, J.P. May, J.E. McClure, and M. Steinberger, $H_{\infty}$ ring spectra and their applications, Lecture Notes in Math., vol. 1176, SpringerVerlag, New York, 1986. MR 836132 (88e:55001)

[EKMM95] A.D. Elmendorf, I. Kř́ǔz, M.A. Mandell, and J.P. May, Modern foundations for stable homotopy theory, Handbook of algebraic topology, NorthHolland, Amsterdam, 1995, pp. 213-253. MR 1361891 (97d:55016)

[GH04] P.G. Goerss and M.J. Hopkins, Moduli spaces of commutative ring spectra, Structured ring spectra, London Math. Soc. Lecture Note Ser., vol. 315, Cambridge Univ. Press, Cambridge, 2004, pp. 151-200. MR 2125040 (2006b:55010)

[HKR00] M.J. Hopkins, N.J. Kuhn, and D.C. Ravenel, Generalized group characters and complex oriented cohomology theories, J. Amer. Math. Soc. 13 (2000), no. 3, 553-594 (electronic). MR 1758754 (2001k:55015)

[Hov08] M. Hovey, Morava E-theory of filtered colimits, Trans. Amer. Math. Soc. 360 (2008), no. 1, 369-382 (electronic). MR 2342007 (2008g:55007)

[HS99] M. Hovey and N.P. Strickland, Morava K-theories and localisation, Mem. Amer. Math. Soc. 139 (1999), no. 666, viii+100. MR 1601906 (99b:55017)

[Kas98] T. Kashiwabara, Brown-Peterson cohomology of $\Omega^{\infty} \Sigma^{\infty} S^{2 n}$, Quart. J. Math. Oxford Ser. (2) 49 (1998), no. 195, 345-362. MR 1645564 (2000d:55013) 
THE CONGRUENCE CRITERION FOR POWER OPERATIONS IN MORAVA E-THEORY 379

[Knu73] D. Knutson, $\lambda$-rings and the representation theory of the symmetric group, Lecture Notes in Math., vol. 308, Springer-Verlag, New York, 1973. MR 0364425 (51 \#679)

[Lei04] T. Leinster, Higher operads, higher categories, London Mathematical Society Lecture Note Series, vol. 298, Cambridge University Press, Cambridge, 2004. MR 2094071 (2005h:18030)

[Lur07] J. Lurie, Derived algebraic geometry II: noncommutative algebra (2007), available at arXiv:math/0702299v5.

[Mac71] S. MacLane, Categories for the working mathematician, Vol. 5, SpringerVerlag, New York, 1971. Graduate Texts in Math. MR 0354798 (50 \#7275)

[Rez08] C. Rezk, Power operations for Morava E-theory of height 2 at the prime 2 (2008), available at arXiv:0812.1320 (math.AT).

[Str97] N.P. Strickland, Finite subgroups of formal groups, J. Pure Appl. Algebra 121 (1997), no. 2, 161-208. MR 1473889 (98k:14065)

[Str98] _ Morava E-theory of symmetric groups, Topology 37 (1998), no. 4, 757-779. MR 1607736 (99e:55008)

[Str99] _ Correction to: "Morava E-theory of symmetric groups" [Topology 37 (1998), no. 4, 759-779; MR1607736 (99e:55008)], Topology 38 (1999), no. 4, 931. MR 1679805 (2000a:55010)

[Swe74] M.E. Sweedler, Groups of simple algebras, Inst. Hautes Études Sci. Publ. Math. (1974), no. 44, 79-189. MR 0364332 (51 \#587)

[Tak77] M. Takeuchi, Groups of algebras over $A \otimes \bar{A}$, J. Math. Soc. Japan 29 (1977), no. 3, 459-492. MR 0506407 (58 \#22151)

[Voe03] V. Voevodsky, Reduced power operations in motivic cohomology, Publ. Math. Inst. Hautes Études Sci. (2003), no. 98, 1-57. MR 2031198 (2005b:14038a)

[Wil82] C. Wilkerson, Lambda-rings, binomial domains, and vector bundles over C $P(\infty)$, Comm. Algebra 10 (1982), no. 3, 311-328. MR 651605 (83f:55003)

Charles Rezk rezk@math.uiuc.edu

Department of Mathematics, University of Illinois, 273 Altgeld Hall, MC-382, 1409 W. Green Street, Urbana, IL 61801 